\title{
Enlarged symmetry algebras of spin chains, loop models, and $S$-matrices
}

\author{
N. Read ${ }^{1}$ and H. Saleur ${ }^{2,3}$ \\ 1 Department of Physics, Yale University, P.O. Box 208120, \\ New Haven, CT 06520-8120, USA \\ 2 Service de Physique Théorique, \\ CEA Saclay, 91191 Gif sur Yvette, France \\ 3 Department of Physics and Astronomy, \\ University of Southern California, \\ Los Angeles, CA 90089, USA
}

(Dated: January 11, 2007)

\begin{abstract}
The symmetry algebras of certain families of quantum spin chains are considered in detail. The simplest examples possess $m$ states per site $(m \geq 2)$, with nearest-neighbor interactions with $\mathrm{U}(m)$ symmetry, under which the sites transform alternately along the chain in the fundamental $m$ and its conjugate representation $\bar{m}$. We find that these spin chains, even with arbitrary coefficients of these interactions, have a symmetry algebra $\mathcal{A}_{m}$ much larger than $\mathrm{U}(m)$, which implies that the energy eigenstates fall into sectors that for open chains (i.e., free boundary conditions) can be labeled by $j=0,1, \ldots, L$, for the $2 L$-site chain, such that the degeneracies of all eigenvalues in the $j$ th sector are generically the same and increase rapidly with $j$. For large $j$, these degeneracies are much larger than those that would be expected from the $\mathrm{U}(m)$ symmetry alone. The enlarged symmetry algebra $\mathcal{A}_{m}(2 L)$ consists of operators that commute in this space of states with the Temperley-Lieb algebra that is generated by the set of nearest-neighbor interaction terms; $\mathcal{A}_{m}(2 L)$ is not a Yangian. There are similar results for supersymmetric chains with $\mathrm{gl}(m+n \mid n)$ symmetry of nearest-neighbor interactions, and a richer representation structure for closed chains (i.e., periodic boundary conditions). The symmetries also apply to the loop models that can be obtained from the spin chains in a spacetime or transfer matrix picture. In the loop language, the symmetries arise because the loops cannot cross. We further define tensor products of representations (for the open chains) by joining chains end to end. The fusion rules for decomposing the tensor product of representations labeled $j_{1}$ and $j_{2}$ take the same form as the Clebsch-Gordan series for $\mathrm{SU}(2)$. This and other structures turn the symmetry algebra $\mathcal{A}_{m}$ into a ribbon Hopf algebra, and we show that this is "Morita equivalent" to the quantum group $U_{q}\left(\mathrm{sl}_{2}\right)$ for $m=q+q^{-1}$. The open-chain results are extended to the cases $|m|<2$ for which the algebras are no longer semisimple; these possess continuum limits that are critical (conformal) field theories, or massive perturbations thereof. Such models, for open and closed boundary conditions, arise in connection with disordered fermions, percolation, and polymers (self-avoiding walks), and certain non-linear sigma models, all in two dimensions. A product operation is defined in a related way for the Temperley-Lieb representations also, and the fusion rules for this are related to those for $\mathcal{A}_{m}$ or $U_{q}\left(\mathrm{sl}_{2}\right)$ representations; this is useful for the continuum limits also, as we discuss in a companion paper.
\end{abstract}

\section{INTRODUCTION}

\section{A. Motivation}

For many years there has been wide interest in lattice and continuum quantum field theory systems in two space-time (or two Euclidean space) dimensions in areas ranging from statistical mechanics to high-energy theory (including string theory). The lattice models include those like the six-vertex model [1], and when a continuum limit is taken in one of the two dimensions (call it time) in an appropriate manner, the transfer matrix becomes a local quantum-mechanical Hamiltonian acting on a space of states which for many lattice models is of the form of a quantum spin chain, that is a tensor product of vector spaces, one for each site in the chain. In the most interesting cases, the continuum limit in the second dimension, or in both dimensions simultaneously, can also be taken to obtain a continuum quantum field theory. This requires that the original lattice or spin chain model be at or close to a critical point. In this limit, attention is focused on the low-energy and long-wavelength states of the system. The quantum field theory description, including correlation functions, then yields the universal properties of the underlying lattice model. In cases of interest here, the quantum field theory of the nearby critical point possesses conformal symmetry, which in two dimensions is potentially very powerful in its analysis. At the same time, other "internal" (non-spacetime) symmetries that may be present in the lattice model play an important role as they survive in the continuum theory. Then a knowledge of the theory at a critical point is also a starting point for the analysis of massive quantum field theories obtained by applying a relevant perturbation (the integral over space-time of a local operator) to the conformal one.

Many lattice systems and conformal field theories (and their massive deformations) have been understood for some time. But there remain others that are not understood, and which have been of recent interest in various 
diverse contexts:

(i) Among spin chains, there are the $m, \bar{m}$ chains with $\mathrm{SU}(m)$ symmetry, in which the fundamental representation, denoted $m$, of $\mathrm{SU}(m)$ alternates with its dual (or conjugate) representation $\bar{m}$ along the chain, and the Hamiltonian is the unique nearest-neighbor $\mathrm{SU}(m)$ invariant ("Heisenberg exchange") coupling. While the $m=2$ case is the usual spin- $1 / 2$ chain, the cases $m>2$ have not been well understood, but are of interest in connection with valence-bond theories of high temperature superconductors, for example. In the $m>2$ cases, only first-order phase transitions occur.

(ii) Very similar spin chains, but with $\mathrm{SU}(m)$ replaced by the Lie superalgebra sl(2|1), appeared in connection with a lattice model that exemplifies a variant of the quantum Hall transition, termed the spin Hall transition [2]. In these cases, there is a second-order phase transition which was shown to have some of the same exponents as ordinary classical percolation [2]. Understanding this example of a localization transition in two dimensions is of great interest for localization physics generally.

(iii) There are various "loop models" that occur in statistical mechanics, in which each configuration is a collection of loops in two dimensions, which are assigned Boltzmann weights that depend on the number of loops, the lengths of the loops, whether the loops intersect, and so on [3]. The loops may be a subset of the edges of some lattice, or may be in the continuum. Problems such as polymers (self-avoiding random walks) and percolation have formulations as loop models (see e.g. Refs. $[1,4,5])$. These problems are among a subclass in which the loops are required never to cross themselves or each other. They are related also to stochastic Loewner evolution (SLE).

(iv) There is a family of similar spin chain models generalizing those in (ii) with Lie superalgebras as symmetries, and crucially, the partition functions of all these models can be expanded as sums over sets of non-crossing loops as in point (iii) (this is how the relation with percolation was found [2, 6, 7] ). For these theories the complete exact spectrum of the conformal field theory (CFT) at the critical point has been found [8] , and exhibited surprisingly large multiplicities that increased exponentially with energy (or scaling dimension). Also, the natural massive perturbation has been described at the level of S-matrices [9]. These theories may also be viewed as critical points in other continuum quantum field theories, such as non-linear sigma models that possess the same Lie superalgebras as global symmetries.

(v) The CFTs in points (iii), (iv) are examples of irrational, logarithmic CFTs. The majority of the CFTs that are understood are what are known as rational theories, in which the local fields in the theory fall into a finite set of representations of some so-called chiral algebra, such as the Virasoro algebra [10]. In irrational theories, this fails (though numbers such as the scaling dimensions and central charge may still be rational numbers). Moreover, the semisimplicity property (full decomposability of oper- ator products into direct sums of irreducible representations, present in the rational case) of the representations of the chiral algebra that occur is generally lost, and this can lead to logarithms in correlation functions [11]. This class of CFTs is of general interest, in view of applications such as those already mentioned. We note that logarithmic CFTs are non-unitary theories - the inner product on the space of states is necessarily indefinite.

(vi) Irrational CFTs also occur in string theory, and in particular we mention strings moving on a curved space such as anti-de Sitter (AdS) space. These spaces have Lie superalgebras as symmetries and are very similar to those mentioned above. (In string theory applications, non-unitarity of the worldsheet CFT is not necessarily a problem, due to the imposition of the Virasoro constraints.) In some recent work, some of the same nonlinear sigma models with Lie superalgebra symmetry as in (iv) have been considered using S-matrices [12], in the hope of reaching a better understanding of the AdS/CFT correspondence. In these the scattering events are naturally pictured in space-time using loops, as in the loop models. In these models, parameter values at which crossings of loops are absent are special points.

In this paper we return to our study of these problems, and concentrate here on symmetry aspects. (Apart from a brief discussion, extensions to CFT will be left for a separate paper [13].) We exhibit enlarged symmetry algebras of a class of spin chains, loop models, and Smatrices, for both open and closed boundary conditions in the "space" direction. We aim to show how this can contribute to understanding this class of models, at both the lattice and continuum (CFT) levels.

\section{B. A simple example}

Before surveying the rest of the paper, we will give a simple example to show the flavor of the results; this example is presented in detail (in the form of a spin chain) in the following section. For the example, we consider identical particles (of mass $M$ say), moving on a line (with open boundary condition). Each particle carries an internal "spin" space consisting of $m$ possible states, which thus form an $m$-dimensional vector space. When the particles collide, they scatter with some amplitudes, most details of which will not be needed (the symmetry is independent of these other details). The important point is that the in- and out-going spin states of the pair of scattering particles are related in a simple way. To describe it, we will view the particles as always being reflected in the collision, so that the particles labelled by $i$ are identified by their order along the line, with coordinates $x_{i}$, subject to $x_{i} \leq x_{i^{\prime}}$ for all $i<i^{\prime}$. In a collision of $i, i+1$, the spin states afterwards are either the same as before, or if the spins are the same, $a_{i}=a_{i+1}$, then they can instead annihilate and be replaced by another pair $a_{i}^{\prime}=a_{i+1}^{\prime}$ (with coefficients independent of $\left.a_{i}, a_{i}^{\prime}\right)$. That is, the scattering matrix is some amplitude (depending 
generally on the momentum of $i, i+1)$ times the identity matrix in spin space, plus another amplitude times the projection operator $P_{i}$ onto the $\mathrm{O}(m)$ singlet state for the pair of spin states on $i, i+1$; here $P_{i}^{2}=P_{i}$. [In the following sections we use notation $e_{i}=m P_{i}$ instead.] The processes can be represented in a spacetime diagram with time plotted vertically by continuous lines that represent particles, with the spin state flowing along the line. In a collision the particle worldlines reflect off each other (touch) without crossing; the first amplitude can be represented by the lines that approach but have an avoided crossing, and continue upward, while for the second the two incoming lines can be joined as if they annihilate, and a new pair of spin states created for the outgoing particles (that is, the same as the first amplitude but rotated by $\pi / 2$ ). Thus the continuity of the lines shows the global conservation of the spin. A general spacetime multiparticle scattering process, or a contribution to the partition function (in imaginary time) is then represented by a collection of non-crossing lines and closed loops, which may touch but cannot cross.

The model possesses more symmetry than the global $\mathrm{O}(m)$. It has global $\mathrm{U}(m)$ symmetry, if we view the leftmost particle $(i=0)$ as transforming in the fundamental representation of $\mathrm{U}(m)$, and the particles as alternating between the fundamental and its conjugate (or dual) representation thereafter. That is because the only process of annihilation occurs between neighbors, which are now always of the opposite type. This $\mathrm{U}(m)$ symmetry has been noticed repeatedly in the literature in contexts similar to this one. In fact, if the particles are viewed as fixed on lattice sites and interact via the Hamiltonian $H=-\sum_{i} P_{i}$, which has the spin structure of this form, then we obtain the $m, \bar{m}$ models described under point (i) above. Let the generators of the Lie algebra $\mathrm{gl}_{m}$ of $\mathrm{U}(m)$ acting on the $i$ th particle be denoted $J_{i a}^{b}$ (details are given in the next section). Then we will show that the Hamiltonian $H$ commutes with a set of symmetry operators, that are a certain linear subspace of the operators

$$
\widetilde{J}_{b_{1} b_{2} \ldots b_{k}}^{a_{1} a_{2} \ldots a_{k}}=\sum_{0 \leq i_{1}<i_{2}<\cdots<i_{k}} J_{i_{1} b_{1}}^{a_{1}} J_{i_{2} b_{2}}^{a_{2}} \cdots J_{i_{k} b_{k}}^{a_{k}}
$$

for $k=1,2, \ldots$ The subspace is defined by conditions involving all pairs of neighbors $J_{i}, J_{i+1}$ in this expression; the operators in the subspace have components $J_{b_{1} b_{2} \ldots b_{k}}^{a_{1} a_{2} \ldots a_{k}}$, with the condition that any contraction of an $a_{l}$ with $b_{l \pm 1}$ vanishes. The symmetry algebra spanned by the $J$ 's is larger than the $\mathrm{U}(m)$ symmetry, which is fully described by the action of arbitrary products of the generators $J_{a}^{b}=\sum_{i} J_{i a}^{b}$. The algebra generated by the latter (an image of what is called the universal enveloping algebra of $\mathrm{gl}_{m}$ ) is a proper subalgebra of the one found here. The energy eigenstates of the system form multiplets of the enlarged symmetry algebra. As the symmetry operators commute with any Hamiltonian (or S-matrix) that has the specified form in its spin dependence, for example $H=-\sum_{i} \lambda_{i} P_{i}$ with arbitrary real parameters $\lambda_{i}$, there are minimal multiplicities that must be possessed by the eigenstates of any such system, which are the dimensions of the irreducible representations of the symmetry algebra. (For particular cases, there can be further "accidental" degeneracies.) These dimensions are given explicitly by

$$
D_{j}=\left(q^{2 j+1}-q^{-2 j-1}\right) /\left(q-q^{-1}\right),
$$

for the $j$ th representation $(j=0,1 / 2,1,3 / 2, \ldots)$, where $q$ is defined by $m=q+q^{-1}$, and are positive integers whenever $m$ is an integer $\geq 2$. When the number of particles is even (odd), $2 j$ must be even (resp., odd). Each such representation can be decomposed into irreducible representations of $\mathrm{SU}(m)$, which are of increasing complexity as $j$ increases. For example, for the $\mathrm{SU}(3)$ case, the numbers are Fibonacci numbers, $D_{j}=F_{4 j+2}=1,3$, $8,21, \ldots$, and these multiplicities were observed in the $3, \overline{3}$ spin chain model some time ago [14].

The enlarged symmetry algebras result directly from the fact that the worldlines of the particles never cross. This allows some "flexibility" to the symmetry transformations, as if $\mathrm{U}(m)$ could act in a position-dependent way, and still commute with the interaction. The algebra is not the Yangian of $\mathrm{U}(m)$. Unlike Yangian symmetries, this symmetry algebra commutes with arbitrary Hamiltonians such as $H$ above. This means the symmetry applies to random non-translationally-invariant spin chain models as well as translationally-invariant ones. Thus integrability of the models, though present in many of those we mentioned earlier, is not required for the enlarged symmetry to be present.

\section{Loop models}

Before describing more of our results, it may be useful to clarify the relations among some loop models (readers more interested in algebra and symmetry can skip this Section on a first reading). In the above example, we began with identical particles and $\mathrm{O}(m)$ symmetry, but then noticed the $\mathrm{U}(m)$ symmetry that is a simple consequence of non-crossing of lines. This involved the use of alternating fundamental and dual representations. In terms of worldlines, the distinction between the two can be encoded by adding an arrow to a line, so that the line represents the fundamental of $\mathrm{U}(m)$ when the arrow is in the positive time direction (when the line is projected to the time axis), and the dual when it is in the negative time direction. However, if the system has a periodic boundary condition in the space direction, then the orientations cannot consistently alternate all along the system if there is an odd number of particles. In that case we would be forced to drop the arrows and view the loops as without an orientation. When studying partition functions of loop models with a periodic boundary condition in both the space and time directions, so that the system is topologically a torus, this of course applies to both the space and time directions (or "fundamental 
cycles" on the torus). There are then two versions of the loop models, independent of any other parameters: the unoriented version, in which loops are viewed in general as carrying no arrow to orient them, and any number, whether even or odd, of loops can wind around either cycle of the torus; and the oriented version, in which all loops carry a fixed orientation that alternates along the intersection points with any cycle on the torus, and the number of lines crossing any such cycle must clearly be even. We emphasize that unlike in some other models of loops, such as the loop formulation of the six-vertex model, in the oriented loops models the orientations of loops are fixed, and not summed over or treated as dynamical variables. The assignment of orientations to all loop configurations can be made unambiguous in the continuum models by the use of a base point and an orientation for the torus (viewed as an orientable manifold). The orientations are then specified by shading the region (bounded by the loops) that contains the base point, and shading other regions alternately so that each loop separates a shaded from an unshaded region, then adding arrows to the loops such that the shaded regions is to the left as one goes in the direction of the arrow - this step uses the orientation of the torus.

The unoriented loop models can be given a lattice formulation in which the loops occupy a subset of the edges of the honeycomb graph (lattice) [3], and no edge is occupied more than once. The partition function of the model is a sum over these allowed loop configurations, weighted with a factor $K$ for each edge occupied by a loop, and a factor $m$ for each loop [3]. For each value of the parameter $m$ in the range $-2<m \leq 2$, these models possess a second-order phase transition between a high-temperature (massive) phase, and a lowtemperature phase that is itself critical (massless). For each $m$ in $-2<m<2$, all points in the low-temperature phase flow to the same fixed point (CFT). There are thus two critical theories of interest: dilute (the critical point) and dense (the low-temperature phase), so-named because the density of loops increases with decreasing temperature. The oriented loops models can be obtained similarly as models of loops on a subset of the edges of the square lattice, respecting rules for shading regions as described in the previous paragraph, such that shading occurs only on plaquettes on one of the two sublattices (this corresponds to a Potts model with dilution [random removal] of the Potts spins, which lie on the shaded plaquettes). The phase diagram structure of the oriented loops models is similar to that of the unoriented loops models. Then as $m$ varies between 2 and -2 , there are four series of models (or CFTs) to study, corresponding to the choices oriented or unoriented, and dilute versus dense. (In Ref. [8], only three of these series were described. The fourth is the oriented dilute series, which contains the exponents of the tricritical Potts models.) The oriented dense loops phases can be obtained by working in the limit in which the loops fill the edges of the square lattice (i.e. no dilution), and that is the formula- tion with which we will begin here, in which the transfer matrix corresponds to the Hamiltonian $H$ of the $m, \bar{m}$ models. The more general models in which not every edge is occupied correspond to models like the simple example above, but with particles restricted to sites in a chain, with the occupation number of each site taking the values 0 or 1 only. Further, the Hamiltonian contains terms that create or destroy particles in $\mathrm{O}(m)$-singlet pairs on neighboring sites. It should be clear that for the purposes of understanding the symmetry, the presence of empty sites, and of single-particle hopping (kinetic energy) and two-particle creation and destruction terms in the Hamiltonian has no effect. Thus the distinction of dilute versus dense can be ignored when analyzing the symmetry. The oriented and unoriented cases turn out to be closely related for open (i.e. free) boundary conditions in the space direction, as we have already seen, but for closed (i.e. periodic) boundary conditions there are some small differences between them that have to be taken into account.

Again, the fact that the allowed configurations of loops in the loop models do not include crossings (either between distinct loops or parts of the same loop) is crucial for the symmetry analysis. It is also a key feature of the physics. One can consider modified models in which crossings are allowed, and are weighted with some small weight. As crossing of two lines violates the rules for orientation, this is allowed only within the unoriented loops models (for the oriented loops models, the simplest allowed crossing involves three lines intersecting at a point). It has been shown [15] that while this perturbation is irrelevant in the dilute (critical) theories for $-2<m \leq 2$, in the dense theories with $-2<m<2$ it is a relevant perturbation, and causes a flow to a different phase which was identified with a Goldstone theory [15]. The enlarged symmetry is broken by this perturbation. On the other hand, in models or field theories in which one can argue that there is a flow to the universality class of one of the non-crossing loops models [8, 9], the enlarged symmetry is present in the infrared fixed point theory, even though it was not present at shorter length scales. It is also present if the infrared fixed point CFT is perturbed by a relevant perturbation that does not involve crossings of loops or otherwise break the symmetry, as in Ref. [9].

\section{Summary of the paper}

Although the picture of loop configurations that we have just described may be the best way to unify the diverse physical applications mentioned earlier, for our purposes the most direct way to analyze the symmetry is algebraic. Symmetry is after all an algebraic concept, and symmetry generators are associated with a cycle (or "time-slice") across the system. Symmetries are defined as commuting with the Hamiltonian (or transfer matrix). In fact, for $H=-\sum_{i} \lambda_{i} P_{i}$, commutation with $H$ for ar- 
bitrary values of the coefficients $\lambda_{i}$ leads us to search for operators that commute with all the operators $P_{i}$ (or $\left.e_{i}=m P_{i}\right)$ considered earlier. The latter operators generate an algebra, which for open boundary conditions is the Temperley-Lieb (TL) algebra [1, 6, 7, 16]. It is then a well-defined algebraic problem to find the algebra of operators in our Hilbert space that commutes with the given algebra. The resulting algebras then are guaranteed to commute with any Hamiltonian constructed from the operators $e_{i}$ by algebraic operations. In the continuum limit (after the models have been defined for $-2<m \leq 2$ ), the TL algebra becomes the Virasoro algebra in the CFT, and we obtain an infinite-dimensional algebra that commutes with the whole of the Virasoro algebra (or with two copies of Virasoro in the closed case), and which is larger than the global Lie superalgebra symmetry with which we began. The symmetry also survives the effect of certain perturbations that render the field theory massive.

For applications of symmetry in physics, one needs more than just an associative algebra. One also needs to be able to analyze tensor products of representations (defined as vector spaces) as themselves representations of the same algebra, and for each representation there should be a corresponding "dual", the tensor product of which with the original representation contains the singlet (trivial) representation (this is used for describing antiparticles, for example). In terms of the algebra, these requirements mean it must possess additional structures that make it into a Hopf algebra. In our case, we find (for $m \geq 2$ open cases) that not only are the irreducible representations of our algebra labelled by integers $j$, just as for $\mathrm{SU}(2)$, but the Clebsch-Gordan series for decomposing tensor products are also the same. There are additional structures of "braiding" and "twist", and all of these agree with properties of the quantum group deformation of the $\mathrm{sl}_{2}$ Lie algebra, denoted $U_{q}\left(\mathrm{sl}_{2}\right)$. More technically, we can say that the symmetry algebra is Morita equivalent to the $U_{q}\left(\mathrm{sl}_{2}\right)$, and we also obtain a version of this for the cases $|m|<2$ in which the algebra is not semisimple.

The knowledge of these symmetry properties of generic Hamiltonians will aid the numerical analysis of these chains, as the problem reduces to diagonalizing a much smaller matrix (of size $d_{j}$ or $\widehat{d}_{j}$ below) within each symmetry sector.

In the following, we begin with the symmetry algebras for the spin chains, first in Section $\amalg$ for open boundary conditions. We obtain the dimensions of the irreducible representations of the symmetry algebras, and explicit formulas for a basis for these algebras, for both oriented and unoriented cases. In Section [II] we do the same for the closed chains. In Section IV we briefly discuss similar results for the Potts model, and in Section $\nabla$ we address the generalization of the spin chains so that they have supersymmetry $\operatorname{gl}(m+n \mid n)$ or $\operatorname{osp}(m+2 n \mid 2 n)$. Readers whose main interest is in $\mathrm{U}(m)$ spin chains can skip these two sections. Then in Section VI we introduce the structures that make the symmetry algebras for the open cases, in the limit as the length $\rightarrow \infty$, into Hopf algebras, for $|m| \geq 2$, and explain the Morita equivalence with $U_{q}\left(\mathrm{sl}_{2}\right)$. In Section VII] we address the issues arising for the (supersymmetric) cases with $|m|<2$ when the algebras involved are not semisimple, and show the Morita equivalence with $U_{q}\left(\mathrm{sl}_{2}\right)$ in these cases also. This section requires more mathematical sophistication than the rest of the paper. In Section VIII we briefly discuss the continuum limit, especially the case when it is a conformal field theory. Finally, there is an Appendix which contains many technical details. In this paper, we concentrate on stating the ideas and results, and do not always give proofs of the statements; many are elementary or can be found in the literature. The technical level increases towards the end, but we hope that the early stages will be widely accessible to physicists. We concentrate on the oriented loop models, but also give some results for the unoriented loops models.

\section{SPIN CHAINS: OPEN BOUNDARY CONDITION}

In this section, we discuss the most basic examples, those of $\mathrm{U}(m)$ spin chains or oriented loops models, and also the unoriented or $\mathrm{O}(\mathrm{m})$ models. We explain the algebraic background that is sufficient for analyzing these cases. We include explicit results for dimensions and symmetry generators.

\section{A. Oriented loops models}

We consider an $\mathrm{SU}(m)$ antiferromagnetic spin chain with open (free) boundary conditions. It consists of $2 L$ sites labelled $i=0, \ldots, 2 L-1$, with an $m$-dimensional complex vector space $V_{i} \cong \mathbf{C}^{m}$ at each site $(\mathbf{C}$ is the field of complex numbers). The states can be represented using oscillator operators $b_{i}^{a}, b_{i a}^{\dagger}$ for $i$ even, $\bar{b}_{i a}, \bar{b}_{i}^{a \dagger}$ for $i$ odd, with commutation relations $\left[b_{i}^{a}, b_{j b}^{\dagger}\right]=\delta_{i j} \delta_{b}^{a}(a$, $b=1, \ldots, m)$, and similarly for $i$ odd. The destruction operators $b_{i}^{a}, \bar{b}_{i a}$ destroy the vacuum state, the daggers indicate the adjoint, and the spaces $V_{i}$ are defined by the constraints

$$
\begin{array}{rll}
b_{i a}^{\dagger} b_{i}^{a}=1 & (i \text { even }) \\
\bar{b}_{i}^{a \dagger} \bar{b}_{i a}=1 & (i \text { odd })
\end{array}
$$

of one boson per site (we use the summation convention for repeated indices of the same type as $a$ ). We define the generators of $\mathrm{U}(m)$ (or in fact of $\mathrm{gl}_{m}$ ) acting in the spaces $V_{i}$ by $J_{i a}^{b}=b_{i a}^{\dagger} b_{i}^{b}$ for $i$ even, $J_{i a}^{b}=-\bar{b}_{i}^{b \dagger} \bar{b}_{i a}$ for $i$ odd, and the commutation relations among the $J_{i}$ s (for each $i$ ) are $i$-independent. Hence the global $\mathrm{gl}_{m}$ algebra, defined by its generators $J_{a}^{b}=\sum_{i} J_{i a}^{b}$, acts in the tensor product $V=\otimes_{i=0}^{2 L-1} V_{i}$ of copies of the fundamental representation 
of $\mathrm{gl}_{m}$ on even sites, alternating with its dual on odd sites, as desired to construct an antiferromagnetic spin chain. Though the U(1) subalgebra of $\mathrm{gl}_{m}$ generated by $J_{a}^{a}$ acts trivially on the chain (and by a scalar on each site), it is often notationally convenient not to subtract this trace from the generators $J_{a}^{b}$.

The $\mathrm{SU}(m)$-invariant nearest-neighbor coupling in the chain is unique, up to additive and multiplicative constants. It is the usual "Heisenberg coupling" of magnetism, and can be written in terms of operators $e_{i}$, defined explicitly as

$$
e_{i}=\left\{\begin{array}{cl}
\bar{b}_{i+1}^{a \dagger} b_{i a}^{\dagger} b_{i}^{b} \bar{b}_{i+1, b}, & i \text { even } \\
\bar{b}_{i}^{a \dagger} b_{i+1, a}^{\dagger} b_{i+1}^{b} \bar{b}_{i b}, & i \text { odd }
\end{array}\right.
$$

The $e_{i}$ 's are Hermitian, $e_{i}^{\dagger}=e_{i}$. Acting in the constrained space $V$, they satisfy [6, 7] the relations [1, 16]

$$
\begin{aligned}
e_{i}^{2} & =m e_{i}, \\
e_{i} e_{i \pm 1} e_{i} & =e_{i}, \\
e_{i} e_{j} & =e_{j} e_{i} \quad(j \neq i, i \pm 1) .
\end{aligned}
$$

We write the parameter $m$ as $m=q+q^{-1}$. The abstract associative algebra over the complex numbers $\mathbf{C}$ generated by unity and the $n-1$ generators $e_{0}, \ldots, e_{n-2}$ that satisfy the relations (6) (and no other relations algebraically independent of these) with parameter $q \in \mathbf{C}$ will be called the Temperley-Lieb (TL) algebra, $\operatorname{TL}_{n}(q)$ (for $n$ either even or odd). The representation we have constructed in the space $V$ is faithful for $m \geq 2$. All algebras in this paper are over $\mathbf{C}$ and are assumed to include unity.

In the space $V$, a much-studied Hamiltonian for a nearest-neighbor antiferromagnetic spin chain is the $m$, $\bar{m}$ model with open (free) boundary conditions,

$$
H=-\epsilon \sum_{i \text { even }} e_{i}-\epsilon^{-1} \sum_{i \text { odd }} e_{i},
$$

where $\epsilon>0$ is a parameter; if $\epsilon \neq 1$, the model is said to have staggered couplings. In the thermodynamic $(L \rightarrow$ $\infty)$ limit a phase transition occurs at $\epsilon=1$, which is first order for $m>2$, second order for $m=2$. More general Hamiltonians for the spin chain can be constructed, using arbitrary elements of the TL algebra, that is any sum of products of generators, perhaps with random coefficients.

There are also vertex models whose transfer matrices are TL algebra elements. These models may be expanded as configurations of loops that run along (and fill) the edges of the square lattice, with avoided crossings at the vertices. This produces a class of loop model. This uses a well-known graphical representation of the TL algebra that has been described by many authors (see e.g. Ref. [1]). In our models, the loops are viewed as oriented, with the fundamental of $\mathrm{gl}_{m}$ running along in the direction of the arrow. We emphasize again that these orientations are fixed, not summed over (they are not dynamical variables). The two ways of conserving directions of arrows on the loops at a vertex represent the action either of $1 \otimes 1$ or of $e_{i}$ for the two sites $i, i+1$ in question. The symmetry algebra we find in this paper determines the multiplicities in the spectrum of any of these models.

The TL algebra arose in studies of the Potts model [1, 16], to which Refs. [6, 7] thus found a relation of the $\mathrm{SU}(m)$ antiferromagnetic spin chains. In terms of the TL generators $e_{i}$, the $Q$-state Potts model has the same Hamiltonian, and the $e_{i}$ obey the TL algebra with $Q=m^{2}$, but here for all non-negative integers $Q[1]$. In the Potts model partition function, the loops are the boundaries of Potts clusters [1]. The possibility of using different representation spaces for a given algebra was a main point of TL [16], and we will have much more to say about this below.

Because of the Hermiticity of the generators $e_{i}$ with respect to the positive-definite inner product on the vector space $V$, it follows that for any element $a$ of the algebra, $a^{\dagger}$ is also an element. If such an algebra is finite dimensional, then it is automatically semisimple, which implies that any of its finite-dimensional modules (representations [17]) is also semisimple, that is fully reducible into a finite direct sum of irreducible representations (simple modules) [18, 19]. Because the representation of the TL algebra in $V$ is faithful for $m \geq 2$, this shows that $\mathrm{TL}_{2 L}(q)$ is semisimple for $m \geq 2$ (in fact, this holds for all $q \geq 1)$ 20, 21, 22].

The general algebraic results that will be used extensively in this paper will now be summarized. In an irreducible representation of dimension $N<\infty$ of an algebra $A, A$ acts as the full matrix algebra $M_{N}(\mathbf{C})$ of all complex $N \times N$ matrices. Any semisimple algebra $A$ is isomorphic to a finite direct product of such algebras, one for each distinct irreducible (we refer to two irreducible representations as distinct if they are not isomorphic): $A=\prod_{j} M_{N_{j}}(\mathbf{C})$, where $j$ runs over the set of isomorphism classes of representations, of dimensions $N_{j}$. In matrix language, this means that the algebra $A$ is isomorphic to the algebra of all block-diagonal complex matrices, where the blocks are $N_{j} \times N_{j}$. It follows that the dimension of $A$ is $\operatorname{dim} A=\sum_{j} N_{j}^{2}$. The commutant $B$ of such an algebra $A$ in a finite-dimensional representation $V^{\prime}$ (the commutant is the algebra of all linear transformations of $V^{\prime}$ that commute with all elements of $A$ ) must also be semisimple, by Schur's lemma. If the $j$ th distinct irreducible representation of $A$ occurs in $V^{\prime}$ with multiplicity $M_{j}$, then the full matrix algebra $M_{M_{j}}(\mathbf{C})$ commutes with $A$ acting in the subspace of $V^{\prime}$ spanned by the copies of the $j$ th irreducible. Assuming that $M_{j}>0$ for all $j$, which means that $A$ is represented faithfully in $V^{\prime}$, then there is a one-one correspondence between the isomorphism classes of irreducible representations of $A$ and $B$, and $B$ is the direct product of algebras $M_{M_{j}}(\mathbf{C})$ (so $\operatorname{dim} B=\sum_{j} M_{j}^{2}$ ). In particular, (i) both algebras have the same number of distinct irreducible representations; (ii) the commutant of $B$ in $V^{\prime}$ is $A$ (that is, the double commutant of $A$, which necessarily contains $A$, is in fact equal to $A$ ); (iii) the space $V^{\prime}$ can be decomposed 
as

$$
V^{\prime}=\bigoplus_{j} \mathbf{C}^{N_{j}} \otimes \mathbf{C}^{M_{j}}
$$

where $\mathbf{C}^{N_{j}}\left(\mathbf{C}^{M_{j}}\right)$ stands for the irreducible representation of $A$ (resp., $B$ ) of dimension $N_{j}$ (resp., $M_{j}$ ). Hence, $\operatorname{dim} V^{\prime}=\sum_{j} N_{j} M_{j}$. Also, (iv) the center of $A$ (i.e. the subalgebra of elements in $A$ that commute with all elements of $A$ ) is also the center of $B$, and both are isomorphic to $\prod_{j} M_{1}(\mathbf{C})$ (i.e. a direct product of one dimensional algebras, one for each $j$, each isomorphic to the complex numbers). This correspondence between representations of $A$ and $B$ is also a simple form of the more general Morita equivalence of algebras (which applies to algebras that are not necessarily semisimple).

We now apply these general results to $\mathrm{TL}_{n}(q)$ acting in $V$, and construct the commutant algebra explicitly. First, we require information about the representations of the TL algebra $\mathrm{TL}_{n}(q)$ (in this paragraph, we allow $n$ to be odd or even). The $m=2(q=1)$ case of $V$ is instructive. This is just the $\mathrm{su}(2)$ spin- $1 / 2$ chain. The $\operatorname{su}(2)$ symmetry commutes with permutations of the sites, and the TL generators are essentially the transpositions of neighbors, which generate the symmetric group $S_{n}$ on $n$ sites. $\mathrm{TL}_{n}(1)$ is isomorphic to the group algebra of $S_{n}$, projected to the space of $S_{n}$ representations that actually occur in $V$. The latter correspond to Ferrers-Young diagrams with $n$ boxes and at most two rows. It follows that the dimension of the $j$ th irreducible representation of $\mathrm{TL}_{n}(1)$, which is the multiplicity of the spin $j$ representation of $\mathrm{su}(2)$ in the chain, is

$$
d_{j}=\left(\begin{array}{c}
n \\
n / 2+j
\end{array}\right)-\left(\begin{array}{c}
n \\
n / 2+j+1
\end{array}\right),
$$

where $n / 2+j$ must be an integer. For $n$ even, $j=0,1$, $\ldots, n / 2$. The sum of the squares of these dimensions is $\sum_{j} d_{j}^{2}=(n+1)^{-1}\left(\begin{array}{c}2 n \\ n\end{array}\right)$, the dimension of the TL algebra [21]. The same Ferrers-Young diagrams correspond to the representations of $\mathrm{su}(2)$ that occur in $V ; 2 j$ equals the difference in the number of boxes in the two rows in the diagram. This well-known decomposition is called [the $\mathrm{su}(2)$ case of] Frobenius-Schur-Weyl duality, and is an example of the correspondence discussed in the previous paragraph. For $q>1$, the irreducible representations of $\mathrm{TL}_{n}(q)$ retain the same dimensions, because the algebra varies continuously with $q[20,21,22]$, and hence the TL algebra also retains the same dimension $\operatorname{dim} \mathrm{TL}_{n}(q)=$ $(n+1)^{-1}\left(\begin{array}{c}2 n \\ n\end{array}\right)$. This formula is valid for all $m$, including $m=0$, as can be readily seen from the diagrammatic definition of TL, in which each element of a linear basis corresponds to a diagram [20, 21, 22].

We now describe the decomposition of our chain $V$ into irreducibles of $\mathrm{TL}_{n}(q)$, for $n=2 L$. We use the following non-orthogonal, but linearly-independent basis states. Each basis state corresponds to a pattern of nested parentheses and dots, such as ()$\bullet(()) \bullet$, with one symbol for each site of the chain $(2 L=8$ in the example). The parentheses must obey the usual typographical rules for nesting, so that each "(" corresponds to exactly one ")". Also, the dots must not be inside of any parentheses. These rules imply that the () pairs consist of one even and one odd site, and that dots are alternately on even and odd sites, starting with an even site at the left. The states in the chain represented by such a diagram are constructed by contracting the sites that correspond to each () pair into an $\mathrm{SU}(m)$ singlet ("valence bond"). For the dots, the state in the tensor product of spaces $\mathbf{C}^{m}$ (each of which corresponds to a dot) must be chosen so that application of the projection operator to the $\mathrm{SU}(m)$ singlet for any two dots that are adjacent (when parentheses are ignored) annihilates the state. Thus, those sites are "non-contractible".

It is easily seen that the TL algebra applied to these basis states does not mix states with different numbers of non-contractible sites. Application of an $e_{i}$ always produces a valence bond at $i, i+1$, together with a rearrangement of some other contractions for sites that were contracted with $i$ or $i+1$ before (if one of $i, i+1$ was a non-contractible dot, it is moved to another position). Thus, the TL generators $e_{i}$ change a pattern to another valid pattern. However, when $i, i+1$ are both non-contractible, $e_{i}$ annihilates the state. The TL algebra never changes the state on the sequence of dots. The number of valid patterns is independent of $m$, and one can use the $m=2$ case to count them; in this case there is an invertible mapping of the space of states, commuting with the action of $\mathrm{su}(2)$, that maps basis states corresponding to valid patterns with $2 j$ dots to those for "standard" Young tableaus with at most two rows, such that the difference in length of the two rows is $2 j$ (a standard tableau is a Ferrers-Young diagram with one of the numbers $1,2, \ldots, n$ inserted in each box, such that the numbers are increasing both to the right along the rows and down the columns). Hence for each number $2 j=0$, $2, \ldots, 2 L$ of dots, the number of valid patterns coincides with the dimensions $d_{j}$ of $S_{2 L}$ representations [23]. The basis states are linearly independent and span the $j$ th irreducible representation of $\mathrm{TL}_{2 L}(q)$.

The number of states for each valid pattern with $2 j$ dots determines the dimension $D_{j}$ of the $j$ th representation of the commutant of $\mathrm{TL}_{2 L}(q)$ in $V$. These numbers can be found inductively, by adding another pair of noncontractible dots to the end of a sequence, and are independent of $L$. This leads easily to the recurrence relation 8]

$$
D_{1} D_{j}=D_{j+1}+D_{j}+D_{j-1} \text {. }
$$

Also, it is clear that $D_{0}=1, D_{1}=m^{2}-1\left[D_{1}\right.$ is the dimension of the adjoint representation of $\mathrm{SU}(m)]$. Using $m=q+q^{-1}$, the solution is

$$
D_{j}=[2 j+1]_{q}
$$

where $[n]_{q}=q^{n-1}+q^{n-3}+\ldots+q^{-n+1}=\left(q^{n}-q^{-n}\right) /(q-$ $\left.q^{-1}\right)$ is the $q$-deformation of any integer $n$. As a check, the total number of linearly-independent states we con- 
structed is

$$
\sum_{j=0}^{L} D_{j} d_{j}=\left(q+q^{-1}\right)^{2 L}=m^{2 L},
$$

which is exactly $\operatorname{dim} V$. Note that these dimensions are the multiplicities of energy eigenvalues for the generic Hamiltonians in the TL algebra, mentioned earlier. For $m>2$, the dimensions $D_{j}$ asymptotically increase exponentially with $j$. For example, for $m=3$, the first few are $1,8,55,377, \ldots$, and are the Fibonacci numbers $D_{j}=F_{4 j+2}$. The $m=3$ cases were found for Hamiltonian (77) previously [14]. For $j>1$, the decomposition of these multiplets into irreducible representations of $\mathrm{su}(\mathrm{m})$ become increasingly complicated.

To construct the commutant algebra explicitly, we introduce the operators (for $k \leq 2 L$ )

$$
\widetilde{J}_{b_{1} b_{2} \ldots b_{k}}^{a_{1} a_{2} \ldots a_{k}}=\sum_{0 \leq i_{1}<i_{2}<\cdots<i_{k} \leq 2 L-1} J_{i_{1} b_{1}}^{a_{1}} J_{i_{2} b_{2}}^{a_{2}} \cdots J_{i_{k} b_{k}}^{a_{k}}
$$

(for $k=0$, we define $\widetilde{J}=1$, and for $k=1, \widetilde{J}_{b}^{a}=J_{b}^{a}$ as defined earlier). For each $k=0,1, \ldots$, these span a space of dimension $m^{2 k}$. In this space of operators we can impose linear conditions, that the contraction of one of the indices $a$ with a neighboring index $b$ [i.e. of $a_{l}$ with $b_{l+1}$ (resp., $b_{l-1}$ ), for $l=1,2, \ldots, k-1$ (resp., $l=2$, $\ldots, k)]$ is zero. This gives us a basis set $J_{b_{1} \ldots b_{k}}^{a_{1} \ldots a_{k}}$, that are "traceless" in this sense. For example, for $k=2$, we have

$$
J_{b_{1} b_{2}}^{a_{1} a_{2}}=\widetilde{J}_{b_{1} b_{2}}^{a_{1} a_{2}}-\frac{1}{m} \widetilde{J}_{b_{1} a}^{a a_{2}} \delta_{b_{2}}^{a_{1}}-\frac{1}{m} \widetilde{J}_{b b_{2}}^{a_{1} b} \delta_{b_{1}}^{a_{2}}+\frac{1}{m^{2}} \widetilde{J}_{b a}^{a b} \delta_{b_{2}}^{a_{1}} \delta_{b_{1}}^{a_{2}}
$$

and these span a space of dimension $\left(m^{2}-1\right)^{2}$. In general, the dimension is $\left(D_{k / 2}\right)^{2}$. The exact forms are

$$
J_{b_{1} b_{2} \ldots b_{k}}^{a_{1} a_{2} \ldots a_{k}}=\left(P^{\bullet} P \bullet \widetilde{J}\right)_{b_{1} b_{2} \ldots b_{k}}^{a_{1} a_{2} \ldots a_{k}},
$$

where $P^{\bullet}\left(P_{\bullet}\right)$ is the (Jones-Wenzl) projection operator to the "traceless" sector on the vector space indexed by $\left(a_{1}, b_{2}, \ldots\right)$ [resp., $\left.\left(b_{1}, a_{2}, \ldots,\right)\right]$, which can be constructed recursively using the $\mathrm{TL}_{k}(q)$ algebra in these spaces [24].

One can readily show that: (i) all $J_{b_{1} b_{2} \ldots b_{k}}^{a_{1} a_{2} \ldots a_{k}}$ commute with all the $e_{i}$, hence with all of $\mathrm{TL}_{2 L}(q)$ (they leave the patterns unchanged); (ii) all $J_{b_{1} b_{2} \ldots b_{k}}^{a_{1} a_{2} \ldots a_{k}}$ with $k>2 j$ annihilate the $j$ th irreducible representation of the commutant algebra; (iii) the space of $J_{b_{1} b_{2} \ldots b_{k}}^{a_{1} \ldots a_{k}} \mathrm{~S}$ with $k=2 j$ acts as the matrix algebra $M_{D_{j}}(\mathbf{C})$ on the $j$ th irreducible representation; (iv) $J_{b_{1} b_{2} \ldots b_{k}}^{a_{1} a_{2} \ldots a_{k}}$ with $k<2 j$ map the $j$ th irreducible representation into itself, and hence in that subspace can be written as linear combinations of those with $k=2 j$. In particular, in our chain of $2 L$ sites, the operators with $k$ odd are linear combinations of those with $k$ even. Hence only even $k$ are needed. These results show that the algebra spanned by $J_{b_{1} b_{2} \ldots b_{k}}^{a_{1} a_{2} \ldots a_{k}}(k=0$, $2, \ldots)$ is the commutant algebra $\mathcal{A}_{m}(2 L)$ of $T L_{2 L}(q)$ in $V$, with dimension $\operatorname{dim} \mathcal{A}_{m}(2 L)=\sum_{j}\left(D_{j}\right)^{2}$. Because the dimensions $D_{j}$ are independent of $L$, the limit $L \rightarrow \infty$ exists, and we write $\mathcal{A}_{m}=\lim _{L \rightarrow \infty} \mathcal{A}_{m}(2 L)$.

The "obvious" global symmetry algebra is $\mathrm{gl}_{m}$, or more accurately the universal enveloping algebra (UEA) $U\left(\mathrm{gl}_{m}\right)$ of $\mathrm{gl}_{m}$, which is the associative algebra generated by the generators $J_{a}^{b}$ of $\mathrm{gl}_{m}$, subject to the commutation relations of $\mathrm{gl}_{m}$ [or similarly for $\left.U\left(\mathrm{sl}_{m}\right)\right]$ 25, 26]. For $m>2$, our algebra $\mathcal{A}_{m}$ is strictly larger than $U\left(\mathrm{sl}_{m}\right)$; $U\left(\mathrm{sl}_{m}\right)$ is a proper subalgebra of $\mathcal{A}_{m}[27$, and hence the representations of $\mathcal{A}$ can be decomposed into representations of $\mathrm{sl}_{m}$. The dimension of $\mathcal{A}_{m}(2 L)$ can be found in closed form, and grows exponentially with $L$ :

$$
\begin{aligned}
\operatorname{dim} \mathcal{A}_{m}(2 L) & =\frac{q^{4 L+4}-q^{-4 L-4}}{\left(q-q^{-1}\right)^{2}\left(q^{2}-q^{-2}\right)}-\frac{2(L+1)}{\left(q-q^{-1}\right)^{2}}(6) \\
& =\frac{[2 L+2]_{q^{2}}-(2 L+2)}{\left(q-q^{-1}\right)^{2}} \\
& \sim \frac{q^{4 L}}{\left(1-q^{-2}\right)\left(1-q^{-4}\right)}
\end{aligned}
$$

as $L \rightarrow \infty$; here we used $q>1$. By contrast, the dimension of the quotient of $\mathrm{sl}_{m}$ that acts faithfully in the chain is the sum of squares of the dimensions of irreducibles that occur, and the latter dimensions are known polynomials in the highest weight of the representation, of degree at most $m(m-1) / 2$ (the dimensions of irreducibles of $\mathrm{sl}_{m}$ are found by Frobenius-Schur-Weyl duality, and given by the Weyl dimension formula). The highest weights that occur are bounded by something of order the length $L$ of the chain. We have not made a precise estimate of the dimension of the resulting associative algebra, but it is clear that it is bounded by a polynomial in $L$, and thus much smaller than $\mathcal{A}_{m}(2 L)$ for large $L$.

We do not know of a "small" or "simple" set of generators for $\mathcal{A}_{m}$ (that would be analogous to the set of $J_{b}^{a}$ for $\left.U\left(\mathrm{gl}_{m}\right)\right)$. $\mathcal{A}_{m}$ is not the Yangian of $\mathrm{sl}_{m}$. However, the properties above imply that $\mathcal{A}_{m}(2 L)$ is a cellular algebra for all $L$, for which we have given a cellular basis $J_{b_{1} b_{2} \ldots b_{k}}^{a_{1} a_{2} \ldots a_{k}}(k=0,2, \ldots)$, in the sense defined in Ref. [28] (for an exposition, see e.g. Ref. [29], or Sec. VII below). This fact also generalizes to the unoriented and supersymmetric versions below.

\section{B. Unoriented loops models}

The unoriented loops models [3] were discussed in the Introduction. We consider an open spin chain in which each site is in the vector representation of $\mathrm{O}(\mathrm{m})$ [or of the Lie algebra $\mathrm{so}_{m}$ of $\left.\mathrm{O}(m)\right]$. Such a chain can be represented by using oscillator operators $b_{i}^{a}, b_{i a}^{\dagger}$, with commutation relations $\left[b_{i}^{a}, b_{j b}^{\dagger}\right]=\delta_{i j} \delta_{b}^{a}(a, b=1, \ldots, m)$ for all $i, j=0, \ldots, n-1$. (In this subsection, we again allow $n$ to be odd or even.) The destruction operators $b_{i}^{a}$ destroy the vacuum state, and the spaces $V_{i} \cong \mathbf{C}^{m}$ are defined by the constraints

$$
b_{i a}^{\dagger} b_{i}^{a}=1
$$


of one boson per site for all $i$. TL generators can be written, for $i=0,1, \ldots, n-2$, as

$$
e_{i}=\eta^{a b} \eta_{c d} b_{i a}^{\dagger} b_{i+1, b}^{\dagger} b_{i}^{c} b_{i+1}^{d} .
$$

Here $\eta_{a b}$ and its inverse $\eta^{a b}$ represent the non-degenerate symmetric bilinear form, which can be taken to be $\eta_{a b}=$ $\delta_{a b}$ (the Kronecker delta); the invariance of this form defines the symmetry group $\mathrm{O}(m)$. The generators of the $\mathrm{so}_{m}$ Lie algebra on each site are

$$
G_{i a b}=\eta_{b c} b_{i a}^{\dagger} b_{i}^{c}-\eta_{a c} b_{i b}^{\dagger} b_{i}^{c} .
$$

The interaction of sites $i, i+1$ given by the TL generator $e_{i}$ is not the most general one (up to additive and multiplicative constants) allowed by $\mathrm{O}(m)$ symmetry. It generates only loop configurations in which loops never cross, and it is this feature that admits an enlarged symmetry. If we define $\bar{b}_{i a}=\eta_{a b} b_{i}^{b}, \bar{b}_{i}^{a \dagger}=\eta^{a b} b_{i b}^{\dagger}$ for $i$ odd, then as noted in the Introduction, the operators $e_{i}$, which obey the TL relations (6), are actually invariant under $\mathrm{U}(m)$ (with the odd sites transforming in the dual fundamental). Generators $J_{i a}^{b}$ of $\mathrm{gl}_{m}$ can be defined as in the oriented loops models.

The results for the oriented case now generalize easily to the unoriented case. The label $j$ for the representations now takes values $0,1 / 2,1, \ldots, n / 2$, with $2 j$ odd (even) if and only if $n$ is odd (resp., even). A linear basis for the commutant algebra of $\mathrm{TL}_{n}(q)$ is given by the same operators $J_{b_{1} b_{2} \ldots b_{k}}^{a_{1} a_{2} \ldots a_{k}}$, eq. (15) (with $2 L$ replaced by $n$ in the summations), but now the algebra for $n$ even (odd) is spanned by these operators with $k \leq n$ and $k$ even (resp., odd) only; clearly, the even sector is isomorphic to that for the oriented case with the same $m$. The dimensions of the irreducible representations are again given by $D_{j}=[2 j+1]_{q}$ for $q+q^{-1}=m$. As examples, $D_{1 / 2}=m$ is the dimension of the vector representation of so $_{m}$, while $D_{1}=m^{2}-1$ is that of the adjoint of $\mathrm{sl}_{m}$, and decomposes into the antisymmetric and traceless symmetric tensor irreducible representations of $\mathrm{so}_{m}$. We call the $n \rightarrow \infty$ limit of the commutant algebras $\mathcal{B}_{m}$; it includes operators $J_{b_{1} b_{2} \ldots b_{k}}^{a_{1} a_{2} \ldots a_{k}}$ for all $k$, and has irreducible representations for all $j=0,1 / 2,1,3 / 2, \ldots$.

One could also consider $\mathrm{U}(\mathrm{m})$ chains with an odd number of sites, on which the odd sector of $\mathcal{B}_{m}$ would act. However, this would not fit naturally with the later developments below, unlike the $\mathrm{O}(\mathrm{m})$ models.

\section{CLOSED BOUNDARY CONDITION}

In this section, we perform an analysis similar to that of the previous section for the case of the closed boundary condition. This section can be skipped by readers mainly interested in Hopf algebra structures.

\section{A. Oriented loops models}

We generalize the results to the closed (periodic) version of the $\mathrm{SU}(m)$ spin chain models. The space of states is the same (with an even number $2 L$ of sites), and the TL generators are defined as there, but now there are $2 L$ generators $e_{i}$, which obey the relations (6) with $i \pm 1$ interpreted cyclically, with $i=2 L \equiv 0(\bmod 2 L)$. In addition, there is now an obvious cyclic symmetry of the system. We can introduce an operator $u^{2}$ (with inverse $u^{-2}$ ) which translates any state to the right by 2 sites (so as to be consistent with the distinction of two types of sites carrying dual representations), so $u^{2 L}=1$ (there are no odd powers of $u$, though there are in the unoriented or $\mathrm{O}(m)$ cases). We have $u^{2} e_{i} u^{-2}=e_{i+2}$. These operators generate an algebra.

The precise algebra can be defined abstractly as an algebra of diagrams as for TL, but this time on an annulus (or finite cylinder), in which a general basis element corresponds to a diagram of $2 L$ sites on the inner, and $2 L$ on the outer boundary; the sites are connected in pairs, but only configurations that can be represented using lines inside the annulus that do not cross are allowed [30]. Further, for the oriented loops models, the lines must be orientable, such that the arrows emanate from the even sites and enter the odd sites on the inner boundary, and the reverse for the outer boundary. Multiplication is defined in a natural way on these diagrams, by joining an inner to an outer annulus, and removing the interior sites [30]. We emphasize that whenever a closed loop is produced when diagrams are multiplied together, this loop must be replaced by a numerical factor $m$ (as for the TL algebra), even for loops that wind around the annulus, as well as for those that are homotopic to a point. The algebra is generated by the elements $e_{i}$ and $u^{2}$, and they obey the above relations, which however are not a complete set. (The numerical factor $m$ for winding loops is not a consequence of the stated relations, but a separate assumption.) We call this finite-dimensional "annular" algebra [30] the Jones-TL, or JTL algebra, $\mathrm{JTL}_{2 L}(q)$ [30, 31] (the latter terminology is not standard). It is easily seen that our definitions produce a representation of $\mathrm{JTL}_{2 L}(q)$ in $V$, however it turns out that it is faithful only when $m>2$. For $m=2$, the TL algebra already contains all permutations of the sites, and the extra generators $e_{-1}$ and $u^{ \pm 2}$ acting in $V$ can be expressed in terms of the others. Also, for real $q>0$, the JTL algebra is semisimple only for $q \neq 1$ [30], unlike the TL algebra. We will see that the JTL algebra is much richer than the TL algebra. For other periodic generalizations of the TL algebra, which are infinite dimensional, see e.g. Refs. 32, 33].

On passing from $\mathrm{TL}_{2 L}(q)$ to $\mathrm{JTL}_{2 L}(q)$, some irreducible representations of $\mathrm{TL}_{2 L}(q)$ will combine to form irreducibles of $\operatorname{JTL}_{2 L}(q)$. On the other hand, since we work in the same space $V$, when the algebra becomes larger, its commutant must become smaller, and some irreducible representations of the commutant will break 
into irreducibles of the commutant of $\operatorname{JTL}_{2 L}(q)$. (These remarks assume the algebras involved are semisimple.)

The dimensions of the irreducible representations of $\mathrm{JTL}_{2 L}(q)$ for $q>1$ are known [30]. We construct representations of the JTL algebra using parentheses and dots again, but now parentheses can be paired cyclically, so ) $\bullet(()) \bullet$ ( is a valid pattern (valid patterns may also be defined by drawing them on a disk with the sites on the boundary, and lines within the disk connect contracted sites without crossing, while noncontractible sites can be reached within the disk from one another without crossing a contraction line). Contractions that cross the end of the chain, like one in the preceding example, become pairs of dots if one reverts to the open TL point of view, and so one finds for the number of valid patterns with $2 j$ dots

$$
\widehat{d}_{j}=\sum_{j^{\prime}=j, j+1, \ldots} d_{j^{\prime}}=\left(\begin{array}{c}
2 L \\
L+j
\end{array}\right) .
$$

This is valid for $j>0$. For the $j=0$ case, all contraction lines can be drawn without crossing the $0,2 L-1$ link, so $\widehat{d}_{0}=d_{0}$. [These formulas, which as we will see give the dimensions $\widehat{d}_{j}$ of the irreducible representations of $\operatorname{JTL}_{2 L}(q)$, also show how the representations decompose when considered as representations of the subalgebra $\mathrm{TL}_{2 L}(q)$.]

For the set of valid patterns for each value $j=0,1, \ldots$, $L$, one has a set of non-orthogonal but linearly independent basis states, by again associating a singlet valence bond to each pair () of corresponding parentheses, and for the non-contractible sites (now defined cyclically), states that vanish if one such site is contracted with its neighbor on either side (cyclically). The subspace spanned by these elements is a representation of $\operatorname{JTL}_{2 L}(q)$ and of its commutant $\widehat{\mathcal{A}}_{m}(2 L)$, and its dimension is $\widehat{d}_{j} \widehat{D}_{j}$, where the dimensions $\widehat{D}_{j}$ for each pattern will now be found. By comparing with the definitions for the open case, we see that the dimensions $\widehat{D}_{j}$ obey $D_{j}=\widehat{D}_{j}+D_{j-1}(j \geq 2)$, $D_{1}=\widehat{D}_{1}, D_{0}=\widehat{D}_{0}$. That is,

$$
\widehat{D}_{j}=\left\{\begin{array}{l}
q^{2 j}+q^{-2 j} \\
q^{2}+1+q^{-2} \\
1 \quad(j=0)
\end{array}(j=1),\right.
$$

Note that again $\sum_{j} \widehat{d}_{j} \widehat{D}_{j}=m^{2 L}=\operatorname{dim} V$.

Unlike the open chains, for the closed chains the representations of $\operatorname{JTL}_{2 L}(q) \otimes \widehat{\mathcal{A}}_{m}(2 L)$ of dimension $\widehat{d}_{j} \widehat{D}_{j}$ that we have now constructed for each $j$ are not irreducible when $j \geq 2$. There is a non-trivial center of the restriction of $\operatorname{JTL}_{2 L}(q)$ and of $\widehat{\mathcal{A}}_{m}(2 L)$ to the $j$ th subspace. This may be seen most easily in terms of the commutant $\widehat{\mathcal{A}}_{m}(2 L)$. For any basis state in the $j$ th subspace, the states on the non-contractible sites can be cyclically permuted by moving them two steps to the right, without affecting the pattern. This operation clearly commutes with the JTL algebra, so when viewed as acting on all the basis states simultaneously it gives an operator which lies in the commutant. Further, it commutes with all elements of the commutant (restricted to this subspace), because as we will see in the explicit expressions below, these elements involve sums over position which ensure that they are invariant under these operations on the basis states (ultimately this is because of the isomorphism of the JTL algebra $e_{i} \rightarrow e_{i+1}$ for all $i$ ). Hence this operator lies in the center of the commutant (acting in the $j$ th subspace), and so must also lie in the center of the JTL algebra. By Schur's lemma, it acts as a root of unity $e^{2 i K}$ in any irreducible representation of either algebra. We call $K$ (defined modulo $\pi$ ) the pseudomomentum. As translation of the non-contractible sites by $2 j$ steps brings the state back to itself, we have $j K \equiv 0(\bmod \pi)$. We may conclude that, for each $j \geq 2$, though all irreducible representations of $\mathrm{JTL}_{2 L}(q)$ have the same dimension $\widehat{d}_{j}$, they are not all isomorphic, and there is a distinct irreducible representation for each distinct allowed $K$, and thus $j$ distinct isomorphism classes of irreducibles in all [30].

The representations of the commutant of dimension $\widehat{D}_{j}$ can be decomposed into eigenspaces of $K$, with $K=\pi P / N$ where $P \geq 0$ and $N$ are coprime $(N$ is a divisor of $j$, written $N \mid j$ ). We will denote the dimensions of these subspaces by $\widehat{D}_{j K}$, with $\sum_{K} \widehat{D}_{j K}=\widehat{D}_{j}$, from which again, $\sum_{j, K} \widehat{D}_{j K} \widehat{d}_{j}=m^{2 L}$. For $j=0,1, K \equiv 0$ and $\widehat{D}_{j K}=\widehat{D}_{j}$. When the state on the sequence of $2 j$ non-contractible sites is periodic with period $d, 1 \leq d<j$ (with $d \mid j$ ), it contributes only to pseudomomenta such that $N \mid d$. Using Möbius inversion 34] (similarly to Appendix A of Ref. [8]), we obtain the dimensions $\widehat{D}_{j K}$ of the representations with $j \geq 2$ and given $K$ of the commutant $\widehat{\mathcal{A}}_{m}(2 L)$ of $\mathrm{JTL}_{2 L}(q)$ for $m>2$,

$$
\widehat{D}_{j K}=\sum_{d, d^{\prime}: N \mid d} \frac{\mu\left(d / d^{\prime}\right)}{d}\left(q^{2 d^{\prime}}+q^{-2 d^{\prime}}\right),
$$

where the sum is over all positive divisors $d, d^{\prime}$ of $j$, and $\mu(x)$ is the Möbius function [34]. Alternatively, by calculating the trace of the projection operator onto pseudomomentum $K$ for a fixed pattern, we obtain

$$
\widehat{D}_{j K}=\frac{1}{j} \sum_{r=0}^{j-1} e^{2 i K r}\left[q^{2(j \wedge r)}+q^{-2(j \wedge r)}\right],
$$

where $j \wedge r$ denotes the highest common divisor of $j$ and $r(j \wedge 0=j$ for all integers $j \geq 0)$. These two expressions are equal, again by using Ref. [34]. These multiplicities were given in the second form by Jones [30] (for these oriented cases, we have corrected a small error at the end of Ref. [30]). These representations of $\widehat{\mathcal{A}}_{m}(2 L)$ are irreducible, and the dimension of the algebra is $\operatorname{dim} \widehat{\mathcal{A}}_{m}(2 L)=\sum_{j, K}\left(\widehat{D}_{j K}\right)^{2}$. In the $L \rightarrow \infty$ limit, we obtain an algebra $\widehat{\mathcal{A}}_{m}=\lim _{L \rightarrow \infty} \widehat{\mathcal{A}}_{m}(2 L)$.

If we put $m=2$ (even though this is a case in which the JTL algebra does not act faithfully in $V$ ), the multi- 
plicities correctly vanish whenever $K \not \equiv 0(\bmod \pi)$, but the formula for $\widehat{D}_{j 0}$ for $j>1$ is not correct for this case. Here, because the image of the JTL algebra that acts faithfully in $V$ is the same as TL, its commutant is a quotient of $U\left(\mathrm{sl}_{2}\right)$, with irreducible dimensions $D_{j}=2 j+1$.

Some elements of $\widehat{\mathcal{A}}_{m}(2 L)$ can be constructed as in the open case. We use, for $k \geq 1$,

$$
\widetilde{J}_{b_{1} b_{2} \ldots b_{k}}^{a_{1} a_{2} \ldots a_{k}}=\sum_{i_{1}<i_{2}<\cdots<i_{k}<i_{1}} J_{i_{1} b_{1}}^{a_{1}} J_{i_{2} b_{2}}^{a_{2}} \cdots J_{i_{k} b_{k}}^{a_{k}},
$$

where the summations extend periodically on the chain; these commute with $u^{2}$. A set of elements of the commutant $\widehat{\mathcal{A}}_{m}(2 L)$ can now be written, for $k$ even, as

$$
\widehat{J}_{b_{1} b_{2} \ldots b_{k}}^{a_{1} a_{2} \ldots a_{k}}=\left(P^{\bullet} P_{\bullet} \widetilde{J}\right)_{b_{1} b_{2} \ldots b_{k}}^{a_{1} a_{2} \ldots a_{k}},
$$

where, similarly to the open case, the projector $P^{\bullet}\left(P_{\bullet}\right)$ is the projection operator to the "traceless" sector (annihilated by all $\left.e_{i}\right)$ on the vector space indexed by $\left(a_{1}, b_{2}, \ldots\right)$ [resp., $\left.\left(b_{1}, a_{2}, \ldots,\right)\right]$. The projectors certainly exist, as they project onto (non-irreducible for $k>2$ ) representations of the semisimple algebra $\operatorname{JTL}_{k}(q)$. For $k$ odd, there is another set,

$$
\widehat{J}_{b_{1} b_{2} \ldots b_{k}}^{a_{1} a_{2} \ldots a_{k}}=(P \bullet \widetilde{J})_{b_{1} b_{2} \ldots b_{k}}^{a_{1} a_{2} \ldots a_{k}}
$$

in which $P_{\bullet}^{\bullet}$ is the projection operator to the traceless sector (annihilated by $e_{i}$ ) on the single vector space indexed by $\left(a_{1}, b_{2}, a_{3}, \ldots, a_{k}, b_{1}, a_{2}, \ldots, b_{k}\right)$. These projectors exist in the algebra $\operatorname{JTL}_{2 k}(q)$. (For $k=1, \widehat{J}_{b}^{a}$ is the traceless generator of $\mathrm{sl}_{m}$.) The $\widehat{J}_{\mathrm{S}}$ have the cyclic invariance property,

$$
\widehat{J}_{b_{1} b_{2} \ldots b_{k}}^{a_{1} a_{2} \ldots a_{k}}=\widehat{J}_{b_{k} b_{1} \ldots b_{k-1}}^{a_{k} a_{1} \ldots a_{k-1}}
$$

(the cyclic property is clear for the $\widetilde{J}_{\mathrm{s}}$, and for the $\widehat{J}_{\mathrm{S}}$ with $k$ even uses the fact that the matrix elements of the projectors $P_{\bullet}, P^{\bullet}$ are the same real numbers when written out in the respective bases).

Unlike the open case, for the closed case the $\widehat{J}$ operators with $k$ even do not form a linear basis for $\widehat{\mathcal{A}}_{m}(2 L)$; however, they do generate it. Let us study how they act on the $j, K$ irreducible representations for $k=2 j$. A natural decomposition of this space of $\widehat{J}_{\mathrm{S}}$ is obtained by using the projector $P_{(K)}^{\bullet}\left[\right.$ and $\left.P_{\bullet}^{(-K)}\right]$ onto the subspace of the space indexed by $\left(a_{1}, b_{2}, \ldots\right)$ [resp., $\left.\left(b_{1}, a_{2}, \ldots,\right)\right]$ that has pseudomomentum $K$ (resp., $-K$ ) as well as being annihilated by all $e_{i}$. This is possible because the $\widehat{J}_{\mathrm{S}}$ do preserve pseudomomentum. We note that the cyclic invariance property implies that these operators for $K$ and $-K$ are the same (up to some relabelling). We choose an orthonormal basis for this subspace, indexed by $\alpha, \beta, \gamma, \ldots$ (resp., $\alpha^{*}, \ldots$; there is a correspondence between these bases as indicated by the notation), and write these projected $\widehat{J}_{\mathrm{S}}$ as $\widehat{J}_{\alpha \beta}$. Among the irreducibles with $j=k / 2$, these operators annihilate those with pseudomomentum $\not \equiv K$ or $-K(\bmod \pi)$. Strictly speaking, the cases in which $K \equiv-K(\bmod \pi)$ should be distinguished from the more general cases $K \not \equiv-K(\bmod \pi)$; we will return to this after dealing with the generic case. Then in general one finds that $\widehat{J}_{\alpha \beta}$ maps $\beta$ (with pseudomomentum $K$ ) onto $\alpha$, but also $\alpha^{*}$ (with pseudomomentum $-K)$ to $\beta^{*}$. We may re-normalize such that the first of these non-zero matrix elements is equal to one. Now define operators $\mathcal{J}_{\alpha \beta}=\widehat{J}_{\alpha \gamma} \widehat{J}_{\gamma \beta}$ where $\alpha \neq \beta$, and hence also $\alpha^{*} \neq \beta^{*}$ (the summation convention is not in force for the Greek indices). This is possible because all the spaces for $j>0$ have dimension $>1$. Then we see that $\mathcal{J}_{\alpha \beta}$ acts as the elementary matrix $E_{\alpha \beta}$ (whose only non-zero entry is 1 in position $\alpha, \beta$ ) in the pseudomomentum $K$ representation, and annihilates all others with $j=k / 2$, including that for $-K$. Finally the diagonal entries are defined as $\mathcal{J}_{\alpha \alpha}=\mathcal{J}_{\alpha \beta} \mathcal{J}_{\beta \alpha}$, (for any $\left.\beta \neq \alpha\right)$ which acts as $E_{\alpha \alpha}$ in the $j, K$ irreducible. For the cases in which $K \equiv-K(\bmod \pi)$, one should note that the basis states $\alpha^{*}$ are in the same space, and are essentially a permutation of the basis labelled $\alpha$. $\mathcal{J}_{\alpha \beta}=\widehat{J}_{\alpha \gamma} \widehat{J}_{\gamma \beta}$ acts as $E_{\alpha \beta}$ only if $\alpha^{*}, \beta^{*}$ and $\gamma$ are all distinct. This works provided the space has dimension $>2$, which they all do (for $j>0$ ). As the elementary matrices form a linear basis for the full matrix algebra $M_{\widehat{D}_{j K}}(\mathbf{C})$, we have shown that the operators $\widehat{J}$ do generate the algebra $\widehat{\mathcal{A}}_{m}(2 L)$.

Thus finally, we have defined a set of operators $\mathcal{J}_{\alpha \beta}$ for all even $k$ and for all allowed $K(\bmod \pi)$, which are a linear basis for $\widehat{\mathcal{A}}_{m}(2 L)$, and which act on the corresponding $(j=k / 2)$ irreducible as elementary symmetric matrices, while annihilating those with the same $j$ but different $K$, as well as those with $j<k / 2$. Once again, these properties imply that this basis is cellular [28, 29].

\section{B. Unoriented loops models}

For the unoriented loops models, the only differences in the algebra (for which we will use the, again not standard, notation uJTL) are that the number of sites $n$ can be odd, and that there is now an element $u$ (and its inverse $u^{-1}$ ) that is a translation by one site to the right. This annular algebra $\mathrm{uJTL}_{n}(q)$ was also analyzed by Jones [30]. The dimensions of its irreducible representations are again given by the same formula (22) for $\widehat{d}_{j}$ (with $2 L$ replaced by $n), j \leq n / 2$, where $n / 2+j$ must be an integer.

The description of the center of the algebra is the same as before, except that now translation of the states on non-contractible sites by one step to the right is possible, and we define the eigenvalue of this operation to be $e^{i K}$, where $K$ (now defined modulo $2 \pi$ ) is the pseudomomentum. It obeys $2 j K \equiv 0(\bmod 2 \pi)$. Then we can write $K=2 \pi P / N$, where $P \geq 0$ and $N$ are coprime, and $N \mid(2 j)$. The dimensions $\widehat{D}_{j K}^{(\mathrm{u})}$ of the irreducible representations of the commutant $\widehat{\mathcal{B}}_{m}(n)$ of $\mathrm{uJTL}_{n}(q)$ in $V$ again obey $\sum_{K} \widehat{D}_{j K}^{(\mathrm{u})}=\widehat{D}_{j}$, and we note that for all odd 
$2 j \geq 1, \widehat{D}_{j}=q^{2 j}+q^{-2 j}$. The dimensions $\widehat{D}_{j K}^{(\mathrm{u})}$ are now given for $j \neq 0,1$ by 30 ]

$$
\widehat{D}_{j K}^{(\mathrm{u})}=\frac{1}{2 j} \sum_{r=0}^{2 j-1} e^{i K r}\left[q^{(2 j) \wedge r}+q^{-(2 j) \wedge r}\right] .
$$

(Again, these in fact depend only on the denominator $N$ in $K$.) It is interesting to interpret the small $j$ cases, while giving the result for $j=1 . j=0$ (with $K \equiv 0$ by definition) is the singlet. For $j=1 / 2$, in which case $K \equiv 0$ only, we have dimension $\widehat{D}_{1 / 2,0}^{(\mathrm{u})}=\widehat{D}_{1 / 2}=m$, which is clearly the (irreducible) vector representation of the $\mathrm{so}_{m}$ Lie algebra. For $j=1$, there are now two possibilities, $K \equiv 0$ and $K \equiv \pi(\bmod 2 \pi)$. The dimensions are $\widehat{D}_{10}^{(\mathrm{u})}=m(m+1) / 2-1$ and $\widehat{D}_{1 \pi}^{(\mathrm{u})}=m(m-1) / 2$, which are the dimensions of the traceless symmetric, and the antisymmetric (or adjoint), irreducible representations of $\mathrm{so}_{m}$, respectively. For $j>1$, the irreducible representations of $\widehat{\mathcal{B}}_{m}(n)$ are reducible as representations of $\mathrm{so}_{m}$.

On passing to the $\mathrm{JTL}_{2 L}(q)$ subalgebra of $\mathrm{uJTL}_{n}(q)$ $(n=2 L)$, for which $j$ must be integer, $K$ and $K+\pi$ become identified, because only translations $u^{2}$ are in the algebra. The corresponding pairs of irreducibles of $\widehat{\mathcal{B}}_{m}(2 L)$ combine into a single irreducible of $\widehat{\mathcal{A}}_{m}(2 L)$, and addition of the dimensions, $\widehat{D}_{j K}^{(\mathrm{u})}+\widehat{D}_{j, K+\pi}^{(\mathrm{u})}=\widehat{D}_{j K}$, reproduces eq. (25) (by redefining $r \rightarrow r / 2$ ). It follows that $\widehat{\mathcal{B}}_{m}(2 L)$ is a proper subalgebra of $\widehat{\mathcal{A}}_{m}(2 L)$.

Some elements of the commutant $\widehat{\mathcal{B}}_{m}(n)$, can be found, using the expressions found in the oriented case, together with the same notation as in the unoriented open chains. For $n$ even, the operators $\widehat{J}_{b_{1} b_{2} \ldots b_{k}}^{a_{1} a_{2} \ldots a_{k}}$ (for all $k$ ) still commute with all $e_{i}$, but do not commute with $u$. This can be rectified by considering the combinations $\widehat{G}_{b_{1} b_{2} \ldots b_{k}}^{a_{1} a_{2} \ldots a_{k}}=\widehat{J}_{b_{1} b_{2} \ldots b_{k}}^{a_{1} a_{2} \ldots a_{k}}+u \widehat{J}_{b_{1} b_{2} \ldots b_{k}}^{a_{1} a_{2} \ldots a_{k}} u^{-1}$, which commute with $u$ (using the fact that $u^{2}$ commutes with all $\left.\widehat{J}_{b_{1} b_{2} \ldots b_{k}}^{a_{1} a_{2} \ldots a_{k}}\right)$. The effect of this may be understood as follows. In writing the operators $J_{i a}^{b}$ for the unoriented loops models, a choice was made [which was not forced by the underlying $\mathrm{O}(\mathrm{m})$ symmetry of the models] that the even sites transform as the fundamental of $\mathrm{gl}_{m}$, while the odd sites transform as the dual. The simultaneous translation of all $k$ operators using conjugation by $u$ reverses this assignment. Thus for example, for $k=1$, it reduces to $\widehat{G}_{b}^{a}=\widehat{J}_{b}^{a}+u \widehat{J}_{b}^{a} u^{-1}=G_{b}^{a}$, where $G_{b}^{a}=\eta^{a c} \sum_{i} G_{i b c}$, the generators of global $\mathrm{so}_{m}$, as one would expect. If we lower indices using $\eta_{a b}$,

$$
\widehat{G}_{a_{1} \ldots a_{k}, b_{1} \ldots b_{k}}=\eta_{b_{1} c_{1}} \cdots \eta_{b_{k} c_{k}} \widehat{G}_{a_{1} \ldots a_{k}}^{c_{1} \ldots c_{k}}
$$

and use a multi-index notation $A$ (resp., $B$ ) to stand for the list $\left(a_{1}, a_{2}, \ldots, a_{k}\right)$ (resp., $\left.\left(b_{1}, \ldots, b_{k}\right)\right)$, then we have explicitly,

$$
\begin{aligned}
\widehat{G}_{A, B}= & \eta_{b_{1} c_{1}} \cdots \eta_{b_{k} c_{k}} \widehat{J}_{a_{1} \ldots a_{k}}^{c_{1} \ldots c_{k}} \\
& +(-1)^{k} \eta_{a_{1} c_{1}} \cdots \eta_{a_{k} c_{k}} \widehat{J}_{b_{1} \ldots b_{k}}^{c_{1} \ldots c_{k}} .
\end{aligned}
$$

The operators thus have an additional (anti-) symmetry property

$$
\widehat{G}_{B, A}=(-1)^{k} \widehat{G}_{A, B} .
$$

This generalizes the antisymmetry of $G_{a b}$ to all $k$, and resembles a property of the UEA $U\left(\mathrm{so}_{m}\right)$ of $\mathrm{so}_{m}$, that an product of generators $G_{a_{1} b_{1}} \cdots G_{a_{k} b_{k}}$ has the same (anti)symmetry. Hence this space of operators is smaller than that for the oriented loops models.

For $n$ odd, if we try to construct corresponding operators $\widehat{G}_{b_{1} b_{2} \ldots b_{k}}^{a_{1} a_{2} \ldots a_{k}}$, we notice that the assignment of sites as alternately even and odd cannot be performed consistently on the odd chain, though it can be if we use a double cover. Consequently, the summations over sites (which are needed to ensure commutation with $e_{i}$ ) which must extend around the chain cyclically, automatically reverse the assignment of sites as fundamental and dual when an operator $J_{i a}^{b}$ is carried around the system. This leads to a modified cyclic invariance property

$$
\widehat{G}_{b_{k} a_{1} \ldots a_{k-1}, a_{k} b_{1} \ldots b_{k-1}}=-\widehat{G}_{a_{1} \ldots a_{k}, b_{1} \ldots b_{k}}
$$

for $n$ odd (for $n$ even it has the same form as for the $\widehat{J}_{\mathrm{S}}$ in the oriented case). When all $k$ operators are carried once around the system (by $i \rightarrow i+n$ ), all assignments are reversed, and this already ensures commutation with $u$. That is, for each set of $i_{1}, \ldots, i_{k}$ taking values in $0,1, \ldots$, $n-1$ that are allowed by the cyclic ordering inequalities as in the expression for $\widetilde{J}$, there are two terms, instead of one as in $\widetilde{J}$ for $n$ even. Thus for example for $k=1$, this again produces just $G_{a b}$. For the resulting operators $\widehat{G}_{A, B}$, the conditions are that the contractions

$$
\begin{aligned}
\eta^{a_{l} b_{l+1}} \widehat{G}_{a_{1} \ldots a_{l} \ldots a_{k}, b_{1} \ldots b_{l} \ldots b_{k}} & =\eta^{a_{l+1} b_{l}} \widehat{G}_{a_{1} \ldots a_{l} \ldots a_{k}, b_{1} \ldots b_{l} \ldots b_{k}} \\
& =0
\end{aligned}
$$

for $l=1, \ldots, k-1$, but for $l=k$ we have

$$
\eta^{a_{k} a_{1}} \widehat{G}_{a_{1} \ldots a_{k}, b_{1} \ldots b_{k}}=\eta^{b_{1} b_{k}} \widehat{G}_{a_{1} \ldots a_{k}, b_{1} \ldots b_{k}}=0
$$

for all $k$, unlike the $n$ even case (this follows from the others using the modified cyclic invariance). These can be implemented using projectors of the types $P \bullet$ for $k$ even, $P \cdot P^{\bullet}$ for $k$ odd - the reverse of the $n$ even case. The (anti-)symmetry property (33) for all $k$ holds here also and is another consequence of the modified cyclic invariance. Note that explicit expressions for all the operators $\widehat{G}_{A, B}$ for all $n$ and $k$ can be obtained by using $J \mathrm{~s}$ for the unoriented open chains, and taking linear combinations so as to impose the additional conditions [ (modified) cyclic invariance and contractions] listed here.

The odd sector includes the $j=1 / 2$ representation, the vector representation of $\mathrm{so}_{m}$, and the algebra $\widehat{\mathcal{B}}_{m}(n)$ acts in this representation as $M_{m}(\mathbf{C})$, which is not spanned by the generators $G_{a b}$ (the antisymmetric matrices), though they do generate it algebraically. They also generate the full matrix algebra for each of the two $j=1$ irreducible 
representations. We expect that the $\widehat{G}_{A, B} \mathrm{~S}$ (ranging over all $k$ ) generate $\widehat{\mathcal{B}}_{m}(n)$, similarly to the oriented case, but here with $k$ even for the even sector, $k$ odd for the odd sector.

\section{POTTS MODELS}

This short section addresses corresponding results for (the transfer matrix of) the Potts models, or the Hamiltonian of the so-called quantum Potts models. It is not needed for the remainder of this paper.

The preceding results for the open, oriented loops models also hold for the Potts representation of the TL algebra, for $Q=m^{2}$ any positive integer. In the Potts representation, each site in the chain on which the Potts transfer matrix acts corresponds to two sites of the spin chain, and there are $Q$ states for each such Potts site. The obvious global symmetry is the permutation (or symmetric) group $S_{Q}$, which has only a finite set of isomorphism classes of irreducibles. The commutant of the (open) TL algebra in these representations is generally larger and for $Q \geq 4$ (when the open symmetry algebras are semisimple), possesses arbitrarily many irreducibles as $L \rightarrow \infty$. The dimensions of the irreducible representations are given by the same expressions as $D_{j}$ in terms of $q$, where now $q^{2}+q^{-2}+2=Q$. Note that these dimensions are integers whenever $Q$ is one. When $Q$ is a square, $Q=m^{2}$, the symmetry algebras are isomorphic to those in the spin chains above, for $Q \geq 4$, as follows from Refs. 6, 7]. (We note that the proof of the isomorphism of the Potts chain to the $m, \bar{m} \mathrm{U}(m)$ spin chain in Refs. [6, 7] can be completed easily if one uses the algebraic perspective as in the present paper.) For $Q=4$, the symmetry algebra of the open Potts chain is just $U\left(\mathrm{sl}_{2}\right)$ (projected to the integer spins). For $Q<4$, the TL algebras are not represented faithfully in the Potts representation; instead, some semisimple quotient algebra [20] is represented faithfully. For $Q<4$ the symmetry algebras are smaller, more like the group algebra of $S_{Q}$.

The closed cases require further study, even in the cases $Q>4$. There is a loop representation of the Potts partition function (see e.g. Ref. [1]) on the torus, but the weights have to be modified according to certain topological properties of the clusters (shaded regions enclosed by a loop) [4]. It follows that the algebra generated by the $e_{i}$ s and the translation $u^{2}$ in the Potts representation is not isomorphic to the JTL algebra [32, 33]. While the $e_{i}$ s and the translation $u^{2}$ still obey the periodic version of relations (6) and $u^{2} e_{i} u^{-2}=e_{i+2}$, we do not have the complete set of relations that defines whatever algebra they generate. Hence we have not established an algebraic analog of the modified weights in the partition function, but it will change the boundary condition built into the JTL algebra (and only the boundary condition, as we know that the subalgebra associated to any open portion of the chain is a TL algebra). This most likely changes the symmetry (commutant) algebra. For exam- ple, for $Q=4$, one knows that the CFTs for the continuum limit of the chains with uniform coefficients, are different in the two cases, the periodic spin- $1 / 2$ chain and the periodic $Q=4$ Potts model, though they are closely related. In particular, the $Q=4$ periodic Potts model does not have $\mathrm{SU}(2)$ symmetry, even in the continuum limit.

\section{SUPERSYMMETRIC SPIN CHAINS}

This section briefly describes the results for the supersymmetric generalizations of the spin chains. The detailed constructions are given in the Appendix, and are essential for Sec. VII] where the nonsemisimple cases $m<2$ of the open chains are considered. It is not needed for the following section on the ribbon Hopf algebra structure of the $\mathrm{U}(m)$ chains.

The spin chains for the oriented loops models can be generalized so that each site carries a $\mathbf{Z}_{2}$-graded vector space of dimensions $m+n$ for the even (bosonic), $n$ for the odd (fermionic), subspace ( $n \geq 0$ is an integer). This space is the fundamental of the Lie superalgebra $\operatorname{gl}(m+n \mid n)$ for $i$ even, and its dual for $i$ odd. The chain is the graded tensor product of these $V_{i}$ (it may be constructed [8] using fermion operators $f_{i a}^{\dagger}, \bar{f}_{i}^{a \dagger}$ for $a=m+n+1, \ldots, m+2 n$, while $a=1, \ldots, m+n$ corresponds to boson operators as in the $n=0$ special case; details are given in the Appendix). The $(\mathrm{J}) \mathrm{TL}$ algebra is again generated by operators $e_{i}$ (and $u^{2}$ ). These models exist for all integer $m$, provided $m+n, n \geq 0$ [35], and are non-trivial when $m+2 n>1$. The phase transition properties, including scaling dimensions, are the same independent of $n$, though some multiplicities may vanish for small $n$. Even though the finite-dimensional representations of $\operatorname{gl}(m+n \mid n)$ are not always semisimple, the representations of $\mathrm{TL}_{2 L}(q)$ and its commutant $\mathcal{A}_{m+n \mid n}(2 L)$ are still semisimple for $|m| \geq 2$, and similarly for $\operatorname{JTL}_{2 L}(q)$ and its commutant $\widehat{\mathcal{A}}_{m+n \mid n}(2 L)$ for $|m|>2$. (The commutant algebras here are actually superalgebras when $n>0$; details about graded tensor products and superalgebras can be found in the Appendix.) The notation involving $m, n$ for these chains will be used consistently from here on.

For the semisimple cases, the preceding constructions can be carried through for all $n \geq 0$, with only minor variations. The dimensions of the irreducible representations of the commutants can be generalized to the total (usual) dimension, and the superdimension (sdim) which is the dimension of the even (bosonic) subspace minus the dimension of the odd (fermionic) subspace. The superdimensions will now be denoted $D_{j}$ or $\widehat{D}_{j K}$, as they are determined by $m$ alone (in fact, by $m^{2}$ ), independent of $n$, and are given by the same formulas as above, which were the $n=0$ special cases. The total dimensions will be denoted $D_{j}^{\prime}$ or $\widehat{D}_{j K}^{\prime}$, and involve also $q^{\prime}$ determined by $m+2 n=q^{\prime}+q^{\prime-1}$. For the open case, the total 
dimensions are given by the same form $D_{j}^{\prime}=[2 j+1]_{q^{\prime}}$ [8]. For the closed case, total dimensions can be obtained by calculating the trace of the projection operator onto pseudomomentum $K$ for a fixed pattern. One must be careful of minus signs that arise when an odd state of a segment of the non-contractible sites is translated around the system. For $j>1$, the total dimensions for $|m|>2$ are

$$
\widehat{D}_{j K}^{\prime}=\frac{1}{j} \sum_{r=0}^{j-1} e^{2 i K r} w(j, j \wedge r)
$$

where $j \wedge r$ denotes the highest common divisor of $j$ and $r(j \wedge 0=j$ for all integers $j \geq 0)$, and

$$
w(j, d)=\left(q^{2 d}+q^{-2 d}\right) \delta_{j / d \equiv 0}+\left(q^{2 d}+q^{-2 d}\right) \delta_{j / d \equiv 1},
$$

where again $d \mid j$, and the congruences are modulo 2. For $n=0$ this clearly reduces to eq. (25) [30]. The general case can be simplified and (using formulas from Ref. [34]) shown to be equal to the numbers $\Lambda_{\bmod }(M=j, N)$ for these models obtained by a different method in Ref. [8] (up to a continuation to the different range $|m| \leq 2$ studied there). For $j=0,1$, we have $K \equiv 0$ only, and $\widehat{D}_{j 0}^{\prime}=1, q^{\prime 2}+1+q^{\prime-2}$, respectively [the dimensions of the singlet and the adjoint of $\operatorname{sl}(m+n \mid n)]$.

The supersymmetric versions of the unoriented loops models use the defining (vector) representation of $\operatorname{osp}(m+2 n \mid 2 n)$ on all the sites. As for the $\mathrm{O}(m) \bmod -$ els, these representations are self-dual, so the models still make sense for arbitrary numbers $L$ of sites, even with periodic boundary conditions. For the open chains, the commutant algebra becomes $\mathcal{B}_{m+2 n \mid 2 n}(L)$, which for $L$ even is isomorphic to $\mathcal{A}_{m+2 n \mid 2 n}(L)$. The superdimensions and total dimensions of the irreducibles (for $|m| \geq 2$ ) are given by the same formulas as for the oriented loops models, but with $j=1 / 2,3 / 2, \ldots$, allowed in addition to non-negative integers. For the closed chains, the commutant is $\widehat{\mathcal{B}}_{m+2 n \mid 2 n}(L)$. The total dimensions (for $|m|>2$ ) are given by formulas similar to those for the oriented case (c.f. formulas for the super-dimensions $\widehat{D}_{j K}$ above), and again are related to the numbers $\Lambda_{\bmod }(M=2 j, N)$ for these models that were obtained in Ref. [8].

These results go far towards explaining the large multiplicities found in the spectrum of scaling dimensions in the conformal field theories of the loop models with the closed boundary condition in Ref. [8]. They are still not a complete explanation because so far we have analyzed only the semisimple cases $|m|>2$, while the conformal cases occur for $|m| \leq 2$. We comment on this in Sec. VII below, but a full analysis for the closed cases is beyond the scope of this paper.

On the other hand, as S-matrix formulations are based on open boundary conditions, the unoriented $m=2$ open case with underlying $\operatorname{osp}(2+2 n \mid 2 n)$ symmetry does describe the enlarged symmetry of the theories in Ref. [12] at the special point ( $\chi=0$ in the notation there) at which the loops do not cross. This point is also the end-point of the construction in Ref. [12], and coincides with the Kosterlitz-Thouless transition point [8].

\section{TENSOR PRODUCTS, DUALS, AND BRAIDING}

In this section we introduce natural operations turning a tensor product of representations of the commutant algebras into representations, and likewise for the dual representations, and also operations of braiding and twist. These turn the commutant algebras into "ribbon Hopf algebras", and we explain the close relation, termed "Morita equivalence", of these to the quantum group $U_{q}\left(\mathrm{sl}_{2}\right)$.

At this stage we have obtained semisimple associative algebras $\mathcal{A}_{m+n \mid n}(2 L)$ with $|m| \geq 2$ for open chains, and $\widehat{\mathcal{A}}_{m+n \mid n}(2 L)$ with $|m|>2$ for closed chains, and their $L \rightarrow \infty$ limits $\mathcal{A}_{m+n \mid n}$ and $\widehat{\mathcal{A}}_{m+n \mid n}$. (We also have algebras $\mathcal{A}_{m}(2 L)$ for the open Potts models for integral $Q=m^{2} \geq 4$, which behave like the $n=0$ cases; the following applies to these also, but we will not explicitly refer to them again.) For finite chains of $2 L$ sites, these possess finite numbers of non-isomorphic irreducible representations. Specializing until further notice to the open chains, these representations are labelled simply by $j=0$, $1, \ldots L$. Thus there is a correspondence between these representations for different values of $n$, and even for different values of $m$. In particular, the special case $n=0$, $m=2$ (the $\mathrm{su}(2)$ spin- $1 / 2$ chain) played a special role. Now we must explain what the role of the spin- $1 / 2$ chain is in the general case $m>2$. We focus on $\mathcal{A}_{m}$, as the extension to the superalgebras, though similar and relatively straightforward, is notationally cumbersome; some of the details are given in the Appendix and in the following section.

As semisimple associative algebras, the representation theory of each $\mathcal{A}_{m}(2 L)$ is completely characterized by the set of isomorphism classes of simple modules (labelled by $j$ ) and their dimensions $D_{j}$ (it is only slightly more complicated for the superalgebra cases $n>0$, in which the modules are graded vector spaces with a superdimension $D_{j}$ as well as a total dimension $D_{j}^{\prime}$ ). [Similarly the TL algebras $\mathrm{TL}_{2 L}(q)$, for which the irreducible dimensions $d_{j}$ are independent of $q$, are hence isomorphic as associative algebras for all $q \geq 1$, for each $2 L$.] For $q \neq 1$, a representation of $\mathrm{TL}_{2 L}(q)$ in a "spin-1/2" chain that consists of a tensor factor of $\mathbf{C}^{2}$ for each site was discovered by TL [16]. The Hamiltonian (7) is the so-called XXZ spin chain, with certain boundary terms (see e.g. Ref. 36]). For $q \neq 1$, it is not invariant under $\mathrm{su}(2)$. Instead, the commutant of $\mathrm{TL}_{2 L}(q)$ in this chain is a finite-dimensional image, call it $U_{q}\left(\mathrm{sl}_{2}\right)^{(2 L)}$, of the quantum group $U_{q}\left(\mathrm{sl}_{2}\right)$ [25, 26], which for now we view simply as an associative algebra; this algebra is also referred to as the $q$-Schur algebra $S_{q}(2 L, 2)$ [29, 37]. [For generic $q$, $U_{q}\left(\mathrm{sl}_{2}\right)$ can be defined by relations among its three generators, which are $q$-dependent deformations of the commu- 
tation relations that define $U\left(\mathrm{sl}_{2}\right)$, such that for $q \rightarrow 1$, $U_{q}\left(\mathrm{sl}_{2}\right)$ becomes $U\left(\mathrm{sl}_{2}\right)$ [25, 26].] Hence $U_{q}\left(\mathrm{sl}_{2}\right)^{(2 L)}$ is semisimple for $q \geq 1$, and its simple modules, labeled by $j=0,1, \ldots, L$, have the same dimensions as for $q=1$, namely $2 j+1$. Then there is a one-one correspondence between simple modules of $\mathcal{A}_{m}(2 L)$ and of $U_{q}\left(\mathrm{sl}_{2}\right)^{(2 L)}$ with the same $j$, induced through their mutual relation to the corresponding modules of the TL algebras $\mathrm{TL}_{2 L}(q)$. For these semisimple algebras, that is the full content of Morita equivalence. (The equivalence is not an isomorphism of the algebras, as for example corresponding simple modules can have different dimensions.) Now we introduce additional structures that turn the $L \rightarrow \infty$ limits of $\mathcal{A}_{m}(2 L)$ and of $U_{q}\left(\mathrm{sl}_{2}\right)^{(2 L)}$ into "ribbon Hopf algebras". For these, the equivalence holds only for algebras with the same value of $m$ or the corresponding $q$.

Frequently when dealing with symmetry in physics, we find more structure than just the representations of an associative algebra. In particular, we may find that a tensor product of two representations (where the tensor product is just the usual one of complex vector spaces, or graded tensor product for graded vector spaces) can also be viewed as a representation of the same algebra. For an arbitrary associative algebra it is not obvious how this would be done in a consistent way [i.e. so that iterated products are associative, that is so that the canonical isomorphism of $\left(\mathcal{V}_{j_{1}} \otimes \mathcal{V}_{j_{2}}\right) \otimes \mathcal{V}_{j_{3}}$ and $\mathcal{V}_{j_{1}} \otimes\left(\mathcal{V}_{j_{2}} \otimes \mathcal{V}_{j_{3}}\right)$ as vector spaces is also an isomorphism of representations of the algebra]. It requires some more structure in addition to the associative algebra.

The relevant additional structure is a so-called comultiplication, which is a linear map from the "symmetry" algebra $\mathcal{A}$ into a tensor product with itself, $\Delta: \mathcal{A} \rightarrow \mathcal{A} \otimes \mathcal{A}$ [25, 26]. As $\mathcal{A} \otimes \mathcal{A}$ can be viewed naturally as an associative algebra, the map $\Delta$ is required to be a homomorphism of associative algebras. Then if $\mathcal{V}_{j_{1}}, \mathcal{V}_{j_{2}}$ are two representations of $\mathcal{A}$, the vector space $\mathcal{V}_{j_{1}} \otimes \mathcal{V}_{j_{2}}$, is in a natural way a representation of $\mathcal{A} \otimes \mathcal{A}$, with the two factors $\mathcal{A} \otimes \mathcal{A}$ acting on the respective factors in $\mathcal{V}_{j_{1}} \otimes \mathcal{V}_{j_{2}}$. Then using the comultiplication, $\mathcal{V}_{j_{1}} \otimes \mathcal{V}_{j_{2}}$ becomes a representation of $\mathcal{A}$, with the element $a \in \mathcal{A}$ acting by $\Delta(a) \in \mathcal{A} \otimes \mathcal{A}$. In order to guarantee associativity of the tensor product, we require co-associativity of the comultiplication. If the image of an arbitrary element $a$ of $\mathcal{A}$ is written in Sweedler's notation [26] as

$$
\Delta(a)=\sum_{(a)} a^{\prime} \otimes a^{\prime \prime}
$$

that is as a linear combination of elements of $\mathcal{A} \otimes \mathcal{A}$, then co-associativity is the requirement that, for all $a$,

$$
\sum_{(a)} a^{\prime} \otimes \Delta\left(a^{\prime \prime}\right)=\sum_{(a)} \Delta\left(a^{\prime}\right) \otimes a^{\prime \prime}
$$

(both sides are elements of the algebra $\mathcal{A} \otimes \mathcal{A} \otimes \mathcal{A}$ ).

Familiar examples in physics include groups and Lie algebras (or more accurately, the corresponding associative algebras, the group algebras and UEAs, respectively). For the case of the su(2) Lie algebra, the comultiplication is the familiar notion of addition of angular momentum, which means that the generators $J_{a}(a=x, y, z)$ of $\mathrm{su}(2)$ acting on a tensor product are to be obtained as

$$
\Delta\left(J_{a}\right)=J_{a} \otimes 1+1 \otimes J_{a}
$$

which is easily seen to be co-associative. With the introduction also of a counit map, which is used to make the one-dimensional vector space $\mathbf{C}$ into a representation of $\mathcal{A}$, and an antipode, which turns the dual vector space $\mathcal{V}^{*}$ of $\mathcal{V}$ into a representation of $\mathcal{A}$, together with some further compatibility requirements, one arrives at the definition of a Hopf algebra [25, 26]. The notion of a symmetry as described by a Hopf algebra embodies most of the usual features of symmetries that arise in physics. Quantum groups are examples of Hopf algebras that are not equivalent to groups or Lie algebras. The quantum group $U_{q}\left(\mathrm{sl}_{2}\right)$ is a deformation of the UEA $U\left(\mathrm{sl}_{2}\right)$ of the Lie algebra $\mathrm{Sl}_{2}$ [25, 26].

Turning to our algebras $\mathcal{A}_{m}$, for the semisimple cases $m \geq 2$, a comultiplication can be defined, by first defining for $\widetilde{J}_{\mathrm{S}}$ :

$$
\widetilde{\Delta}\left(\widetilde{J}_{b_{1} b_{2} \ldots b_{k}}^{a_{1} a_{2} \ldots a_{k}}\right)=\sum_{k_{1}=0,1, \ldots, k} \widetilde{J}_{b_{1} b_{2} \ldots b_{k_{1}}}^{a_{1} a_{2} \ldots a_{k_{1}}} \otimes \widetilde{J}_{b_{k_{1}+1} \ldots b_{k}}^{a_{k_{1}+1} \ldots a_{k}} .
$$

Then the comultiplication is

$$
\Delta\left(J_{b_{1} b_{2} \ldots b_{k}}^{a_{1} a_{2} \ldots a_{k}}\right)=\left(P^{\bullet} P \bullet \widetilde{\Delta}(\widetilde{J})\right)_{b_{1} b_{2} \ldots b_{k}}^{a_{1} a_{2} \ldots a_{k}},
$$

where the projectors $P^{\bullet}, P_{\bullet}$ act in the space spanned by the indices as before. This is exactly what happens to a $J$ acting on a chain of length $2 L=2 L_{1}+2 L_{2}$, if the chain is broken into two pieces of lengths $2 L_{1}, 2 L_{2}$, by dropping the generator $e_{2 L_{1}-1}$. It is clear that $\Delta(\mathcal{A})$ lies in $\mathcal{A}_{m} \otimes \mathcal{A}_{m}$ as required. It can be interpreted as saying that two chains of lengths $2 L_{1}, 2 L_{2}$ can be joined end to end, and their states interpreted as representations of $\mathcal{A}_{m}$ in a natural way. Indeed, this is the algebraic origin of the recursion relation eq. (10) for the dimensions of the irreducibles. Coassociativity of $\Delta$ is easily checked, using simple properties of the projection operators $P_{\bullet}$ and $P^{\bullet}$ under inclusions of chains into chains of longer length. Using arguments similar to those in the recursion relation for the dimensions, it is then not difficult to see that if $\mathcal{V}_{j_{1}}$ and $\mathcal{V}_{j_{2}}$ are irreducible representations, the tensor product decomposes as

$$
\mathcal{V}_{j_{1}} \otimes \mathcal{V}_{j_{2}} \cong \bigoplus_{j=\left|j_{1}-j_{2}\right|}^{j_{1}+j_{2}} \mathcal{V}_{j}
$$

and $\mathcal{V}_{j_{2}} \otimes \mathcal{V}_{j_{1}}$ decomposes the same way. Notice that these "fusion rules" (i.e. the multiplicities with which each simple module appears in a tensor product of two given simple modules) are the same as for $\mathrm{su}(2)$, and that the total dimensions of the two sides are equal. The latter is also 
true for the superdimensions in the superalgebra $\mathcal{A}_{m+n \mid n}$ $(n \geq 0)$ case.

Similarly, the antipode (which is a linear map, but reverses the order of products in $\mathcal{A}_{m}$ ) is defined by

$$
S\left(J_{b_{1} b_{2} \ldots b_{k}}^{a_{1} a_{2} \ldots a_{k}}\right)=(-1)^{k} J_{b_{k} \ldots b_{2} b_{1}}^{a_{k} \ldots a_{2} a_{1}},
$$

and is invertible, $S^{-1}=S$. We note that, on the vector space $V$, which is a tensor product $V=V_{0} \otimes V_{2} \otimes \cdots V_{2 L-1}$ (where the order in the tensor product denotes order in the chain), the dual is naturally order-reversing [26], $V^{*}=V_{2 L-1}^{*} \otimes \cdots V_{0}^{*}$, which (because the fundamental and its dual alternate in $V$ along the chain) is another chain of the same type, and the same applies to the states on the non-contractible sites in the $j$ th representation. The dual pairing on each of $V \otimes V^{*}$ and $V^{*} \otimes V$ (and hence those on $\mathcal{V} \otimes \mathcal{V}^{*}$ and $\mathcal{V}^{*} \otimes \mathcal{V}$, where $\mathcal{V}$ is any representation of $\mathcal{A}_{m}$ ), that is the map from the tensor product to $\mathbf{C}$ that commutes with the action of $\mathcal{A}_{m}$, is then given by the canonical (vector space) dual pairing between the corresponding tensor factors $V_{i}$ in $V$ and and $V_{i}^{*}$ in $V^{*}$. The double dual $\mathcal{V}^{* *}$ can be identified with $\mathcal{V}$ via the canonical vector-space isomorphism (equivalently, the "left" and "right" duals of $\mathcal{V}$ are the same, because $S^{-1}=S$ ). More generally, for the superalgebras $\mathcal{A}_{m+n \mid n}$, the antipode is given below in eq. (52). In these cases $(n>0), \mathcal{V}^{* *}$ is not canonically isomorphic to $\mathcal{V}$, even though $S^{-1}=S$ (see the Appendix), but is still isomorphic.

The comultiplication and antipode on $\mathcal{A}_{m}$ agree with those on $U\left(\mathrm{gl}_{m}\right)$, an image of which is a subalgebra of $\mathcal{A}_{m}$, so that our Hopf algebra is an extension (of an image) of the Hopf algebra $U\left(\mathrm{gl}_{m}\right)$. This is not true for the additional structures to which we turn next.

A braiding structure can be defined on the representations of $\mathcal{A}_{m}$. First, one can obtain a representation of the braid group on the spaces $V$. The braid group can be defined by the generators $\sigma_{i}(i=0,1$, $\ldots, 2 L-2)$ and their inverses $\sigma_{i}^{-1}$ subject to the relations $\sigma_{i} \sigma_{i+1} \sigma_{i}=\sigma_{i+1} \sigma_{i} \sigma_{i+1}$ for $i=0,1,2 L-3$, and $\sigma_{i} \sigma_{i^{\prime}}=\sigma_{i^{\prime}} \sigma_{i}$ for $i^{\prime} \neq i \pm 1$. It can be represented in the TL algebra by writing $\sigma_{i}=i\left(q^{1 / 2}-q^{-1 / 2} e_{i}\right)$, $\sigma_{i}^{-1}=-i\left(q^{-1 / 2}-q^{1 / 2} e_{i}\right)$ [38, 39], where the factor of $i$ in this and similar formulas below is a square root of -1 (note that since the braid-group relations are homogeneous, other representations can be obtained by multiplying all $\sigma_{i}$ by a constant; the reason for our particular choice is explained below). Hence there is an action of the braid group on $V$, which commutes with $\mathcal{A}_{m}(2 L)$ (for $q \rightarrow-1$, the $\sigma_{i}$ reduce to generators of the symmetric group, which obey also $\sigma_{i}^{2}=1$ for all $i$; we explain below why it is $q \rightarrow-1$ here, not +1 ). For $m>2$, this representation of the braid group is not unitary for any choice of the common constant factor in the $\sigma_{i}$ [38].

Next we construct an element $\sigma_{2 L_{1}, 2 L_{2}}$ in the braid group on $2 L=2\left(L_{1}+L_{2}\right)$ sites as

$$
\sigma_{2 L_{1}, 2 L_{2}}=\prod_{i=0,1, \ldots, 2 L_{1}-1}^{\rightarrow}\left[\prod_{i^{\prime}=0,1, \ldots, 2 L_{2}-1}^{\leftarrow} \sigma_{i+i^{\prime}}\right]
$$

where the ordered product of operators $x_{i}$ is defined by $\vec{\prod}_{i=0,1, \ldots, n} x_{i}=x_{0} x_{1} \cdots x_{n}$, and the reverse order in $\overleftarrow{\prod}_{i=0,1, \ldots, n} x_{i}$. Then $\sigma_{2 L_{1}, 2 L_{2}}$ obeys $\sigma_{2 L_{1}, 2 L_{2}} \Delta(a)=$ $\Delta(a) \sigma_{2 L_{1}, 2 L_{2}}$ for any $a \in \mathcal{A}_{m}$, when acting on this chain. $\sigma_{2 L_{1}, 2 L_{2}}$ can be viewed as exchanging the two parts of a chain of length $2 L=2\left(L_{1}+L_{2}\right)$. From this one can obtain isomorphisms (which commute with $\mathcal{A}_{m}$ ) $\sigma_{\mathcal{U}, \mathcal{V}}: \mathcal{U} \otimes \mathcal{V} \rightarrow \mathcal{V} \otimes \mathcal{U}$ for each pair of representations $\mathcal{U}, \mathcal{V}$. With the normalization of the $\sigma_{i}$ specified above, if $\mathcal{U}$ or $\mathcal{V}$ is the one-dimensional representation, then $\sigma_{\mathcal{U}, \mathcal{V}}$ is the identity, and it follows that all these isomorphisms $\sigma_{\mathcal{U}, \mathcal{V}}$ are independent of the length of the chain in which $\mathcal{U}$ or $\mathcal{V}$ appear. These isomorphisms satisfy compatibility requirements, including some related to the braid group relations. Note that $\sigma_{\mathcal{V}, \mathcal{U}} \sigma_{\mathcal{U}, \mathcal{V}}$ is not the identity map when $q>1$. $\sigma_{2 L_{1}, 2 L_{2}}$ maps $\mathrm{TL}_{2 L_{1}}(q) \otimes \mathrm{TL}_{2 L_{2}}(q)$ to $\mathrm{TL}_{2 L_{2}}(q) \otimes \mathrm{TL}_{2 L_{1}}(q)$ (acting on these algebras, which are understood to be subalgebras of $\mathrm{TL}_{2 L}(q)$ in two obvious ways, by conjugation). If we define the flip map $\tau$ on the space for the two chains of total length $2 L$ by $\tau\left(v_{1} \otimes v_{2}\right)=v_{2} \otimes v_{1}$, then we find that $\widetilde{\mathcal{R}}_{2 L_{1}, 2 L_{2}}=\tau \circ \sigma_{2 L_{1}, 2 L_{2}}$ commutes with the action of $\mathrm{TL}_{2 L_{1}}(q) \otimes \mathrm{TL}_{2 L_{2}}(q)$, and hence is an element of $\mathcal{A}_{m}\left(2 L_{1}\right) \otimes \mathcal{A}_{m}\left(2 L_{2}\right)$. As $L_{1}, L_{2} \rightarrow \infty, \widetilde{\mathcal{R}}_{2 L_{1}, 2 L_{2}}$ becomes a "universal R-matrix" $\mathcal{R} \in \mathcal{A}_{m} \otimes \mathcal{A}_{m}$, making the Hopf algebra into a braided Hopf algebra [25, 26].

Similarly, we can define a "twist" map by

$$
\tilde{\theta}_{2 L}=\left(i q^{3 / 2}\right)^{2 L}\left(\prod_{i=0,1, \ldots, 2 L-2}^{\leftarrow} \sigma_{i}\right)^{2 L}
$$

This commutes with $\mathrm{TL}_{2 L}(q)$, and hence lies in $\mathcal{A}_{m}(2 L)$ as well as in $\mathrm{TL}_{2 L}(q)$, so it is in the center of both algebras, and its $L \rightarrow \infty$ limit $\theta$ lies in the center of $\mathcal{A}_{m}$. It gives rise to natural isomorphisms $\theta_{\mathcal{V}}$ on each module $\mathcal{V}$ that obey $\theta_{\mathcal{U} \otimes \mathcal{V}}=\left(\theta_{\mathcal{U}} \otimes \theta_{\mathcal{V}}\right) \sigma_{\mathcal{V}, \mathcal{U}} \sigma_{\mathcal{U}, \mathcal{V}}$. The prefactor $\left(i q^{3 / 2}\right)^{2 L}$ in $\widetilde{\theta}_{2 L}$ represents a factor $i q^{3 / 2}$ for the twist on the fundamental (or its dual) representation $V_{i}$ for all $i$ [which correspond to the spin- $1 / 2$ module for $U_{q}\left(\mathrm{sl}_{2}\right)$ ], and ensures that $\theta$ acts as unity on the one-dimensional module. $\theta$ evaluated on the $j$ th simple module of $\mathcal{A}$ is $\theta_{j}=q^{2 j(j+1)}$ (for $j \geq 0$ an integer), which is related to the Casimir $j(j+1)$ for simple modules of $\operatorname{sl}_{2}\left(\theta_{j}\right.$ is the same as for $U_{q}\left(\mathrm{sl}_{2}\right)$ [40]). The twist maps on modules can alternatively be constructed using the braiding and other maps already mentioned, including the canonical isomorphism between the left and right duals of $\mathcal{V}$ that exists because $S^{-1}=S$. With the twist map included, the braided Hopf algebra becomes a "ribbon" Hopf algebra [25, 26, 40] (our definition of $\theta$ would be called $\theta^{-1}$ in these references). This structure allows the definition of a "quantum trace" of any linear map on a finite-dimensional representation $\mathcal{V}$, and in particular a "quantum dimension" 25, 26, 40, 41]. In the case of $\mathcal{A}_{m}$, the quantum trace and dimension turn out to be simply the ordinary vector-space trace and dimension. For the 
superalgebras, the quantum trace and dimension turn out to be the supertrace and superdimension.

Now we describe Morita equivalence more formally. Morita equivalence of associative algebras means that their "categories of modules" are equivalent (as categories) [19]. The category of modules of an algebra $\mathcal{A}$ consists of all the modules of $\mathcal{A}$, together with all the morphisms (or homomorphisms - linear maps commuting with the action of $\mathcal{A}$ ) between them. Morphisms can be composed to form other morphisms. Equivalence of these categories for two algebras $\mathcal{A}, \mathcal{B}$, means that there is mapping (a covariant functor) $\mathcal{F}$ from the modules of $\mathcal{A}$ to those of $\mathcal{B}$, and from morphisms of $\mathcal{A}$ to those of $\mathcal{B}$, which is compatible with the composition of morphisms. There is also a functor $\mathcal{G}$ in the reverse direction, and the composite of these functors, in either order, is a functor from one of the categories into itself, which is required to be "naturally isomorphic" to the identity functor. "Naturally isomorphic" to the identity means that for each module of $\mathcal{A}$ there is an isomorphism between it and its image under the composite functor $\mathcal{G} \mathcal{F}$, and these are compatible with the morphisms and their images under $\mathcal{G F}$ also. Morita showed that such an equivalence could always be described in a certain canonical form involving a bimodule over $\mathcal{A}$ and $\mathcal{B}$, that is a module over $\mathcal{A}$ and $\mathcal{B}$ simultaneously, such that the actions on it of $\mathcal{A}$ and $\mathcal{B}$ commute [19]. For us, the module $V$ is a suitable choice for this bimodule which yields an equivalence between $\mathcal{A}_{m}(2 L)$ and $\mathrm{TL}_{2 L}(q)$, and likewise the spin-1/2 chain of $2 L$ sites yields the equivalence between $U_{q}\left(\mathrm{sl}_{2}\right)^{(2 L)}$ and $\mathrm{TL}_{2 L}(q)$. Under this equivalence, modules we labeled with the same value of $j$ correspond. The composite of these equivalences yields the desired Morita equivalence. For semisimple algebras, a Morita equivalence is completely determined by a correspondence of simple modules over the respective algebras. This means that in fact as associative algebras, $\mathcal{A}_{m}(2 L)$ and $U_{q}\left(\mathrm{sl}_{2}\right)^{(2 L)}$ for all $m \geq 2$ and all $q \geq 1$ are all Morita equivalent; however, this might not hold when the additional structures of tensor product, duality, braiding, and twist have been introduced. Moreover, for these semisimple associative algebras, there are $(L+1)$ ! distinct Morita equivalences between each pair; the one under which the modules with the same value of $j$ correspond is the only natural choice for our purposes below.

When the tensor product is introduced, the category of modules over $\mathcal{A}_{m}$ becomes a "tensor" (or "monoidal") category. With the duality of modules determined by the antipode, the tensor category becomes a "rigid tensor category". The Morita equivalence (under which modules with the same $j$ correspond) respects the fusion rules and duality, so that the algebras $\mathcal{A}_{m}$ and $U_{q}\left(\mathrm{sl}_{2}\right)$ still appear to correspond for all $m \geq 2$ and all $q \geq 1$, and indeed are still Morita equivalent (equivalence of tensor categories is discussed in Refs. [25, 26, 40]). With the introduction also of the braiding related to the R-matrix, the rigid tensor category becomes a "rigid braided tensor category". With the introduction of the twist, the rigid braided tensor category becomes a "ribbon category" [26, 40, 41]. Our statement in its most refined form is then that for each value of $|m|,|m| \geq 2$, all the ribbon Hopf algebras $\mathcal{A}_{m+n \mid n}$ for $n \geq 0$, and $U_{q}\left(s_{2}\right)$ for $m=q+q^{-1}$ are Morita equivalent, in the sense that their categories of finite-dimensional modules are equivalent ribbon categories. [Here we mean the $U_{q}\left(\mathrm{sl}_{2}\right)$ modules that decompose into integer spins only.] The equivalence given by the use of a suitable bimodule maps all the ribbon category structures to each other functorially. This implies numerical relationships between the structures. For example, the quantum dimensions defined for corresponding modules in each category should be the same. This explains why the dimensions $D_{j}=[2 j+1]_{q}$ of the simple $\mathcal{A}_{m}$ modules (and the superdimensions of the simple $\mathcal{A}_{m+n \mid n}$ modules for $n>0$ ), which are the quantum dimensions for $\mathcal{A}_{m}$ (resp., $\mathcal{A}_{m+n \mid n}$ ), are equal to the wellknown quantum dimensions of the corresponding simple $U_{q}\left(\mathrm{sl}_{2}\right)$ modules. This also shows that the categories for distinct values of $|m|$ are not equivalent as ribbon categories, because the values of these numbers differ.

In viewing the spin- $1 / 2$ chain as a representation of $\mathrm{TL}_{2 L}(q)$ and of $U_{q}\left(\mathrm{sl}_{2}\right)$, it is best to think of it too as alternating the fundamental (spin-1/2) with its dual, and the TL generators $e_{i}$ as constructed using the $U_{q}\left(\mathrm{sl}_{2}\right)$ invariant dual pairing of nearest neighbors. However, for the unoriented $\mathrm{O}(m)$ and $\operatorname{osp}(m+2 n \mid 2 n)$ models, with $m+2 n, n \geq 0$, (and again $|m| \geq 2$ in this paragraph) one is forced to use an isomorphism ( $\phi$, say) of the fundamental to its dual, for example when concatenating two chains when the one on the left has an odd number of sites. Then we must do the same for the spin- $1 / 2$ chain also. Now many representations of the UEAs of certain Lie algebras such as $\mathrm{sl}_{2}, \mathrm{so}_{m}$ and $\mathrm{sp}_{2 m}$ (and we note that $\left.\mathrm{sl}_{2} \cong \mathrm{so}_{3} \cong \mathrm{sp}_{2}\right)$ possess isomorphisms $\phi_{\mathcal{V}}: \mathcal{V}^{*} \rightarrow \mathcal{V}$ to the representation from its dual. This map can be iterated, $\phi_{\mathcal{V} *} \circ \phi_{\mathcal{V}}: V^{* *} \rightarrow \mathcal{V}$, and the latter map is either +1 or -1 times the canonical vector space map $\mathcal{V}^{* *} \rightarrow \mathcal{V}$ on irreducible representations $\mathcal{V}$; these are then called real or pseudoreal, respectively. For $\mathrm{so}_{m}$, all tensor (i.e. non-spinor) representations are real, while for $\mathrm{sl}_{2}$, halfodd-integer spins are pseudoreal. In our $\mathrm{O}(m)$ chains [and hence also in the $\operatorname{osp}(m+2 n \mid 2 n)$ chains], all representations are likewise real in this sense. The definition of real and pseudoreal can be turned into an intrinsic property, called the Frobenius-Schur indicator $\nu_{\mathcal{V}}$, of a ribbon category that possesses maps $\phi_{\mathcal{V}}$. It is equal to \pm 1 on simple modules (or zero when no isomorphism $\phi_{\mathcal{V}}$ exists). It turns out to be -1 for half-integer spins for the standard ribbon category structure on $U_{q}\left(\mathrm{sl}_{2}\right)$, as one would expect from the above discussion of Lie algebras, but is +1 for all simple modules of $\mathcal{B}_{m}$. This is then a discrepancy with which we must deal.

We note that the twist can be redefined without changing the braiding, though the map between right and left duals also changes. For $U_{q^{\prime \prime}}\left(\mathrm{sl}_{2}\right)$ (the relevant value of $q^{\prime \prime}$ will be determined below) and $\mathcal{B}_{m}$, the category of modules is generated by iterating fusion with the $j=1 / 2$ 
module. The properties of $\theta_{j}$ show that we can multiply $\theta_{1 / 2}$ by a constant and that determines the change for all other modules; then the fact that $\theta_{0}=1$, together with the fusion rules, implies that the only possible change is by multiplication by $(-1)^{2 j}$. Thus there are just two possible distinct twists for given duality, braiding maps, etc. As explained in Ref. [42], the essential use of the map $\phi_{\mathcal{V}_{1 / 2}}$ implies that the variant twist is obtained, not the standard one for $U_{q^{\prime \prime}}\left(\mathrm{sl}_{2}\right)$. For this definition, the Frobenius-Schur indicator is +1 for all modules (this difference in connection with TL algebras was noted in Ref. [43]), and thus agrees with that for our algebras $\mathcal{B}_{m+2 n \mid 2 n}$. The twist for a simple module of $\mathcal{B}_{m+2 n \mid 2 n}\left(\widetilde{\theta}_{2 L}\right.$ is defined as above, but now we allow $2 L$ to be odd) works out as $\theta_{j}=i^{4 j^{2}} q^{2 j(j+1)}$, which is $q^{2 j(j+1)}$ for $2 j$ even, $i q^{2 j(j+1)}$ for $2 j$ odd. The quantum dimension of the $j=1 / 2$ module of $U_{q^{\prime \prime}}\left(\mathrm{sl}_{2}\right)$ works out as $-q^{\prime \prime}-q^{\prime \prime-1}$ because of the variant twist, so we must put $q^{\prime \prime}=-q$. Hence we should compare with $U_{-q}\left(\mathrm{sl}_{2}\right)$ with the variant twist (which can be obtained by applying the above construction of $\widetilde{\theta}_{2 L}$ in the spin- $1 / 2$ chain), and for this the values on the simple modules are $\theta_{j}=(-1)^{2 j}(-q)^{2 j(j+1)}=i^{4 j^{2}} q^{2 j(j+1)}$ [using $\left.(-q)^{1 / 2}=i q^{1 / 2}\right]$. This explains the factor $i$ in the braiding $\sigma_{i}$ of $j=1 / 2$ with itself also; the $\sigma_{i}$ s in the $U_{-q}\left(\mathrm{sl}_{2}\right)$ spin- $1 / 2$ chain, when defined as above, define the standard braiding which reduces to the usual exchange (the flip $\tau$ ) when $-q=1$. We conclude that for the unoriented loops models, $\mathcal{B}_{m+2 n \mid 2 n}$ is Morita equivalent as a ribbon Hopf algebra with $U_{-q}\left(\mathrm{sl}_{2}\right)$ with the variant ribbon structure, where as usual $m=q+q^{-1}$. (For the oriented loops models which involve only integer $j$, this reduces to the same as before.)

Returning to the algebras $\mathcal{A}_{m}$ (and $\mathcal{A}_{m+n \mid n}$ ), we now consider a further numerical relationship that follows from these results. The Clebsch-Gordan coefficients for $\mathcal{A}_{m}$, which describe the explicit decomposition of a tensor product of simple modules into simple modules, as in eq. (44), are determined by the comultiplication and not only by the fusion rules. They cannot be expected to correspond for the different algebras as they depend on a basis for each representation, and corresponding representations of $\mathcal{A}_{m}$ for different $m>2$ and those of $U_{q}\left(\mathrm{sl}_{2}\right)$ do not even have the same dimensions. However, $6 j$ symbols [or recoupling coefficients; coefficients that relate alternative ways of constructing a tensor product of three representations, again analogous to those familiar for $\mathrm{su}(2)$ ] can be constructed as suitable sums (over the bases) of products of Clesch-Gordan coefficients, and are independent of a basis. An abstract construction of $6 j$ symbols, using only the structure of a ribbon category, is given in Ref. [41]. Using that formulation, we can show that the $6 j$ symbols for $\mathcal{A}_{m}$ are the same as the quantum $6 j$ symbols [39] that apply for $U_{q}\left(\mathrm{sl}_{2}\right)$ at the corresponding value of $q$.

\section{NON-SEMISIMPLE CASES}

In this section, we turn to the non-semisimple cases $|m|<2(|m| \leq 2)$ of the open (resp., closed) chains in the spaces $V$. The cases $-2<m \leq 2$ are of particular interest as in these the Hamiltonian in eq. (7) with $\epsilon=1$ is at a critical (second-order phase transition) point, and a continuum limit can be taken that produces a conformal field theory (CFT) [2, 8] (the case $m=2, n=0$ is the well-known spin- $1 / 2$ chain; all cases are non-trivial only if $m+2 n>1$, so the remainder require the use of the supersymmetric models). We will again concentrate on the open oriented-loops models. In the nonsemisimple cases, all earlier results and formulas must be reconsidered. We want to know whether the Morita equivalences of the commutants as ribbon Hopf algebras that hold in the semisimple cases also hold in the nonsemisimple ones. We begin by showing that the commutant is a cellular algebra, which produces insight into the structure of the algebra, including a formula for its dimension. Next we establish the Morita equivalence for the finite-dimensional associative algebras in the finitelength chains. Then we discuss carefully the $L \rightarrow \infty$ limits of the TL algebra, its commutant, and of the categories of modules over these algebras. Then we introduce the structures that turn the $L \rightarrow \infty$ commutant algebras $\mathcal{A}$ into ribbon Hopf algebras and establish their Morita equivalence as such with $U_{q}\left(\mathrm{sl}_{2}\right)$. In particular, this involves the interpretation of a product of modules as a module over the commutant, and there is a corresponding product for modules of the TL algebras, and both of these behave stably in the $L \rightarrow \infty$ limit. Finally, we discuss the closed chains, though not in as much detail.

The mathematical background required in this section is larger than in the earlier sections. Accordingly, it will be appropriate to refer to (simple) modules rather than (irreducible) representations.

\section{A. Preliminary remarks}

The TL algebra acts faithfully in the spin- $1 / 2$ chain representation discovered by TL, for all $q$. The delicate cases occur when $q$ is a root of unity (but $q \neq \pm 1$ ), so $q^{r}= \pm 1$ for some integer $r>1$. With $m=q+q^{-1}$ and $m$ integer, $q$ is a root of unity for $m=-1,0,1$. In these cases, the TL algebra acts faithfully in our spaces $V$ provided $m+2 n>1$; it is then not obvious that the TL algebra or its commutant are semisimple, as we are not aware of a faithful representation on a positive-definite space of states. In fact, it is not semisimple, and neither is $U_{q}\left(\mathrm{sl}_{2}\right)$ in these cases. This means that there are reducible modules that are not fully decomposable. Such a module contains a proper submodule, but is not isomorphic to a direct sum of simple modules. Such modules can however be decomposed into a direct sum of indecomposable components; an indecomposable module is one that cannot be decomposed further as a direct sum 
of its submodules. Frequently, the decomposition into indecomposables is unique up to isomorphism.

Here we will address the structure of these chains only at an abstract level, leaving the details of the structure of the modules for the companion paper [13] (for TL and $U_{q}\left(\mathrm{sl}_{2}\right)$, these are available in the literature [22, 25, 36]). These details are used in only a few places in these arguments. We will need to be more aware of the difference between right and left modules than we have been up to now. That is, if $v$ is an element of a right module $V$ over a ring $R$, then an element $a$ of $R$ acts on it as $v a$, and this action obeys $v 1=v, v(a b)=(v a) b$. We note that any right module over an algebra $R$ can be viewed as a left module over the opposite algebra $R^{\text {op }}$ (the opposite algebra is defined in Appendix A). For $\mathrm{TL}_{2 L}(q)$, the opposite algebra is isomorphic to $\mathrm{TL}_{2 L}(q)$ itself, because the defining relations (6) are invariant if the order in all products of elements is reversed (equivalently, there is an anti-isomorphism of TL with itself). Hence there is a (Morita) equivalence between the categories of right and of left modules over TL. (Indeed there is more than one, as the isomorphism with the opposite algebra can be composed with the automorphism of the TL algebra given by $e_{i} \rightarrow e_{2 L-2-i}$ for all $i$.) In particular, this applies to the important class of modules known as projective modules (a module is projective if and only if there is a free module in which it is a direct summand [18, 19, 44]). Further, as the TL algebra is a finitely-generated algebra over the field of complex numbers $\mathbf{C}$, there is a Morita duality between its categories of finitely-generated right and left modules [45]; this duality is similar to Morita equivalence, except that the functors are contravariant instead of covariant - they reverse the direction of morphisms. In the present case, the duality is simply obtained: the dual of any left module, viewed as a vector space, is the dual vector space, on which the TL algebra naturally acts on the right, and similarly for the dual of a right module. In view of the equivalence between the categories of right and left modules, this becomes a duality of the category of finitely-generated left modules into itself. (This is similar to the use of the antipode in a Hopf algebra to view the dual of a left module as another left module.) This duality will be used occasionally in the following. We must note that the dual of a projective left module is not necessarily a projective right module, and vice versa.

\section{B. Cellular structure}

It will be useful here to derive the cellular structure of the commutant algebras $\mathcal{A}$, which will give important insight into their structure [or that of $\left.U_{q}\left(\mathrm{sl}_{2}\right)^{(2 L)}\right]$. The derivation of Morita equivalence with $U_{q}\left(\mathrm{sl}_{2}\right)^{(2 L)}$ in the next subsection then allows the structure of the commutants to be obtained by combining our analysis with the known structure of $U_{q}\left(\mathrm{sl}_{2}\right)^{(2 L)}$ (we go into further detail about this structure in the companion paper [13]).

We will explain the notion of a cellular algebra [28], using the alternative basis-free definition of König and $\mathrm{Xi}[29,46]$. We will use the TL algebra as an example. To show that an algebra $T$ [such as $T=\mathrm{TL}_{2 L}(q)$ ] is cellular, it is sufficient [46] to show the following: there is a nested chain of ideals $T^{(2 j)}$,

$$
0=T^{(-2)} \subset T^{(0)} \subset T^{(2)} \subset \cdots \subset T^{(2 L-2)} \subset T^{(2 L)}=T
$$

and an anti-involution ${ }^{s}$ of $T$ to itself (an anti-involution is an anti-isomorphism that squares to the identity isomorphism), such that the quotients $T^{(2 j)} / T^{(2 j-2)}$, which are ideals of $T^{(2 L)} / T^{(2 j-2)}$, have the following properties (we call the anti-involution ${ }^{s}$ rather than ${ }^{*}$ as in Ref. 28] to avoid possible confusion with the use of ${ }^{*}$ for duals): (i) $\left(T^{(2 j)} / T^{(2 j-2)}\right)^{s}=\left(T^{(2 j)} / T^{(2 j-2)}\right)$ for $j=0, \ldots$, $L$; (ii) for each $j$, there is a left ideal $\mathcal{R}_{j}$ of $T / T^{(2 j-2)}$ such that $T^{(2 j)} / T^{(2 j-2)}$ is isomorphic to $\mathcal{R}_{j} \otimes \mathcal{R}_{j}^{s}$ as a $T / T^{(2 j-2)}-T / T^{(2 j-2)}$ left-right bimodule, compatibly with ${ }^{s}$ (such an ideal is called a cell ideal, while the modules $\mathcal{R}_{j}$ for each $j$ are called cell or standard modules). In the last formula, $\mathcal{R}_{j}^{s}$ is the right module with action of $t \in T^{(2 L)} / T^{(2 j-2)}$ defined by $v t=t^{s} v$, where $v$ is an element of $\mathcal{R}_{j}$ viewed as a vector space. $\mathcal{R}_{j}$ (resp., $\mathcal{R}_{j}^{s}$ ) can be viewed as a left (resp., right) module of $T$ also, which is annihilated by $T^{(2 j-2)}$. For TL, the ideals $T^{(2 j)}(j \geq 0)$ can be described in the usual diagrammatic picture as spanned by the elements that correspond to diagrams with at most $2 j$ lines connecting the top to the bottom rows of dots; these are easily seen to form ideals, as the number of lines is non-increasing under multiplication of diagrams. The anti-involution can be taken as either of the two described above; we construct the more appropriate choice below. Before continuing, we point out that the cell (or standard) modules $\mathcal{R}_{j}$ of TL are also called Specht modules. They have dimension $d_{j}$, and are a generalization of those we constructed in the semisimple case using "valid patterns". For $q$ a root of unity they are indecomposable, but not in general simple modules.

Now we derive the cellular structure of the commutant. As this is a superalgebra, and as the cellular structure refers to the opposite (super-)algebra, we actually need a slight generalization to cellular superalgebras. This uses some definitions given in the Appendix. First, we will obtain the nested chain of ideals in $\mathcal{A}=\mathcal{A}_{m+n \mid n}(2 L)$ :

$$
0 \subset \mathcal{A}^{(2 L)} \subset \mathcal{A}^{(2 L-2)} \subset \cdots \subset \mathcal{A}^{(0)}=\mathcal{A},
$$

with quotients $J^{(k)}=\mathcal{A}^{(k)} / \mathcal{A}^{(k+2)}$ which are ideals of $\mathcal{A} / \mathcal{A}^{(k+2)}$ (for $k$ even; the notation is consistent with earlier usage of $k$ ). These ideals $\mathcal{A}^{(k+2)}$ for $k=0, \ldots$, $2 L-2$ are defined as consisting of endomorphisms of $V$ as a TL-module (i.e., linear maps to itself that commute with the TL algebra) that annihilate all elements of $T^{(k)}$ (from either side, as they commute). That is, $a \in \mathcal{A}$ is a member of $\mathcal{A}^{(k+2)}$ if at $=0$ for all $t$ in $T^{(k)}$. These spaces are easily seen to form ideals in $\mathcal{A}$, and to be nested as required. Explicit relations that define elements in $\mathcal{A}^{(k)}$ 
are given in the Appendix. The elements constructed there for each $k$ are representatives of the cosets forming the quotient spaces $J^{(k)}$, and by abuse of notation we tend to call the spaces of these elements $J^{(k)}$ also. We will refer to basis elements in these spaces loosely as $J_{b_{1} \cdots b_{2 L}}^{a_{1} \cdots a_{2 L}}$, as they correspond to those operators in earlier sections, however caveats about this form of notation are discussed in the Appendix. The construction shows that they lie in $\mathcal{A}^{(k)}$, but we have yet to show that they span it; we turn to this next.

First we consider $\mathcal{A}^{(2 L)}=J^{(2 L)}$, which annihilates the ideal $T^{(2 L-2)}$. Note that $T^{(2 L)} / T^{(2 L-2)} \cong \mathbf{C}$ is one dimensional, and consists of cosets of 1 (the identity) only, while $T^{(2 L-2)} / T^{(2 L-4)}$ is spanned by the cosets of all the $e_{i}$ s. Hence $\mathcal{A}^{(2 L)}$ consists of elements of $\mathcal{A}$ that annihilate the $e_{i} \mathrm{~s}$. This is exactly our set $J_{b_{1} \cdots b_{2 L}}^{a_{1} \cdots a_{2 L}}$, because as $k=2 L$ here, these consist of a $J_{b}^{a}$ for each site of the chain, and the definitions coincide. This space $J^{(2 L)}$ has dimension $\left(D_{L}^{\prime}\right)^{2}$, and superdimension $\left(D_{L}\right)^{2}$, which are given by the same expressions as before, but now for arbitrary $m$.

Now we consider the next case, $k=2 L-2$. We require elements of $\mathcal{A}$ that annihilate all elements of TL with $2 L-4$ through-lines. A subspace of this space $T^{(2 L-4)}$ is spanned by diagrams consisting of $e_{2 L-2}$ (involving the last two sites), and any diagram from the ideal $T^{(2 L-4)}$ for the algebra $\mathrm{TL}_{2 L-2}(q)$ on the first $2 L-2$ sites. The commutant of this subspace can be analyzed in the tensor product space of $V(2 L-2)$ (for the first $2 L-2$ sites) and $V(2)$ (for the last two). The commutant of $e_{2 L-2}$ in the space $V(2)$ for the last two sites consists only of the identity, modulo operators that annihilate $e_{2 L-2}$ (the proof of this works differently for the cases $m \neq 0$ and $m=0)$. Then we can tensor this with elements of $\mathcal{A}^{(2 L-2)}(2 L-2)$ for the first $2 L-2$ sites, and these are the span of $J_{b_{1} \cdots b_{2 L-2}}^{a_{1} \cdots a_{2 L-2}}$ that we have from the previous step, as applied to the length $2 L-2$ chain. The ideal $\mathcal{A}^{(2 L-2)}$ that we seek is a subspace of this space of operators. But in fact, we have such a space of operators that commute with the full $\mathrm{TL}_{2 L}(q)$, and which reduce to this space modulo elements that annihilate $e_{2 L-2}$ (and hence are linearly independent); these are just the $J_{b_{1} \cdots b_{2 L-2}}^{a_{1} \cdots a_{2 L-2}}$ for the length $2 L$ chain. Hence, using induction, we conclude that each $J^{(k)}$ is spanned by the cosets of the elements $J_{b_{1} \cdots b_{k}}^{a_{1} \cdots a_{k}}$.

To complete the cellular structure, and make some basic statements about modules, we need an anti-involution ${ }^{s}$; although one can consider the possibility that ${ }^{s}$ is only an anti-isomorphism, we do find that there is such an anti-involution. First we obtain (details are given in the Appendix) a natural anti-involution of the algebra of endomorphisms of $V$ into itself, $E=$ End $V$. $E$ is naturally isomorphic to $V \otimes V^{*}$. As we have noted previously, for the chain we have $V=V_{0} \otimes V_{1} \otimes \cdots V_{2 L-1}$, and the dual naturally reverses order, so $V^{*}=V_{2 L-1}^{*} \otimes \cdots V_{0}^{*}$. $V^{*}$ is not quite identical to $V$, despite the alternation of $V_{0}$ and its dual $V_{0}^{*}$ along the chain in $V ; V_{0}^{* *}$ is not precisely the same as $V_{0}$, but there is an isomorphism between them.
(In the special case $n=0$ treated earlier, this distinction can be ignored.) The anti-involution is named ${ }^{s}$ because it is so closely related to the supertranspose map which takes an endomorphism $f$ of $V$ to $f^{*}$, an endomorphism of $V^{*}$ (acting on the left on $V^{*}$ ).

In our representation in the chain $V$, both the TL algebra and its commutant $\mathcal{A}$ are subalgebras of $E$, and we expect that ${ }^{s}$ restricted to these subalgebras is the requisite anti-involution. First, it is easily checked from the definitions (again, see the Appendix) that ${ }^{s}$ maps TL to itself: it takes $e_{i} \rightarrow e_{i}^{s}=e_{2 L-2-i}$, so it is the chainreversing anti-involution of TL mentioned above. Then if $a \in \mathcal{A}, t \in \mathrm{TL}$, at $=t a$ implies $a^{s} t^{s}=t^{s} a^{s}$ and hence $a^{s} t=t a^{s}$ for all $t \in \mathrm{TL}$. That is, the image $\mathcal{A}^{s}$ is contained in $\mathcal{A}$, and vice versa, so $\mathcal{A}^{s}=\mathcal{A}$, and so ${ }^{s}$ is an anti-involution of $\mathcal{A}$.

Next we consider the ideals $\mathcal{A}^{(k)}, k=0,2, \ldots, 2 L$. First, we wish to show that $J^{(k)}=\mathcal{A}^{(k)} / \mathcal{A}^{(k+2)}$ are mapped onto themselves by ${ }^{s}$, for all $k$. In fact, the ideals $\mathcal{A}^{(k)}$ are defined as the annihilators of $T^{(k-2)}$ from either side. As these conditions are again mapped to themselves by ${ }^{s}$, we have $\mathcal{A}^{(k) s}=\mathcal{A}^{(k)}$ for all $k$. Finally we want to show that $J^{(k)} \cong \mathcal{V}_{k / 2} \otimes \mathcal{V}_{k / 2}^{s}$ as $\mathcal{A}$ - $\mathcal{A}$-bimodules, where $\mathcal{V}_{j}$ is a left $\mathcal{A}$-module and $\mathcal{V}_{j}^{s}$ is a right $\mathcal{A}$-module related to $\mathcal{V}_{j}$ by using ${ }^{s}$, compatibly with $J^{(k) s}=J^{(k)}$. It will be sufficient to consider simply $J^{(2 L)}=\mathcal{A}^{(2 L)}$ as all $J^{(k)}$ are isomorphic to one of these (for some $L$ ) as ideals of $\mathcal{A} / \mathcal{A}^{(k+2)}$. This is the subspace of $E \cong V \otimes V^{*}$ annihilated by the generators $e_{i}$ of TL on both sides. We note that $E$ itself has the required form, under the anti-involution ${ }^{s}$. Further, we can define $\mathcal{V}_{L}$ to be the subspace of $V(2 L)$ annihilated by all $e_{i}$ (acting on the left), and $\mathcal{V}_{L}^{s}$ to be the subspace of $V(2 L)^{*}$ (viewed as a right module over $E$ ) annihilated by all $e_{i}$ (acting on the right). Hence $J^{(k)} \cong \mathcal{V}_{k / 2} \otimes \mathcal{V}_{k / 2}^{s}$ as $\mathcal{A}$ - $\mathcal{A}$-bimodules, and ${ }^{s}$ maps it to itself. The modules $\mathcal{V}_{j}\left(\mathcal{V}_{j}^{s}\right)$ are the left (resp., right) standard or cell modules for $\mathcal{A}$, and have dimension $D_{j}^{\prime}$, and superdimension $D_{j}$. They correspond under Morita equivalence to the standard modules of $U_{q}\left(\mathrm{sl}_{2}\right)^{(2 L)}$, which are also referred to as Weyl modules, and have dimension $2 j+1$. Again, these standard modules are indecomposable but generally not simple in these non-semisimple cases. Hence the dimension of each space $J^{(k)}$ is $\left(D_{k / 2}^{\prime}\right)^{2}$, and the superdimension is $\left(D_{k / 2}\right)^{2}$. We note that $\mathcal{A} / \mathcal{A}^{(2)} \cong \mathbf{C}$ (given by cosets of 1 ), and $D_{0}^{\prime}=D_{0}=1$. Finally, the total dimension of the commutant $\mathcal{A}$ is $\operatorname{dim} \mathcal{A}=\sum_{j=0}^{L} D_{j}^{\prime 2}$, with similar growth behavior as in the semisimple cases.

Some further properties of the algebra follow from the cellular superalgebra structure 28, 29]. It follows from our construction that $\mathcal{A}(2 L)$ is a quotient of $\mathcal{A}(2 L+2)$ by the ideal $\mathcal{A}^{(2 L+2)}$ of $\mathcal{A}(2 L+2)$. The dimensions of the standard modules are $L$-independent, provided that $j \leq L$. The properties of a standard module $\mathcal{V}_{j}$ can then be found by using the case $L=j$ of $\mathcal{A}(2 L)$. One notices that the inner product on this module can be determined from the multiplication in this algebra, because of the 
isomorphism $\mathcal{A}^{(2 L)} \cong \mathcal{V}_{L} \otimes \mathcal{V}_{L}^{s}$ as $\mathcal{A}$ - $\mathcal{A}$-bimodules. This inner product may be degenerate in the non-semisimple cases of $\mathcal{A}$. There is a submodule of vectors in $\mathcal{V}_{j}$ that are orthogonal to all vectors; the quotient by this submodule is simple, and all isomorphism classes of simple modules are obtained in this way [28, 29]. For our cases $m=0$, \pm 1 (as well as for $|m|>2$ where all standard modules are simple), this simple quotient is non-zero for all $j$. The standard modules of TL can also be analyzed in the same way. Each standard module is either simple (i.e., the submodule mentioned is zero), or contains a simple submodule, such that the quotient module is also simple. It turns out that the simple quotient module is nonzero for all real $m$ except $m=0$, for which the $j=0$ quotient module alone is zero; thus there are less than $L+1$ isomorphism classes of simple modules of TL in this case. For this reason also, the singlet standard module of $U_{q}\left(\mathrm{sl}_{2}\right)^{(2 L)}$ or $\mathcal{A}_{m+n \mid n}(2 L)$ does not appear as a summand in the chain for $m=0$ : its multiplicity is the dimension of the $j=0$ simple TL module, which is zero.

To conclude this subsection, we set our results in a larger context. For $m \neq 0$, the relation between the TL algebra and its commutant is an example of what was studied in the wider context of quasi-hereditary algebras [47], and is now frequently known as "Ringel duality" (see Refs. [29, 48, 49, 50], and Ref. [50] for a pedagogical discussion). (It is not a duality in the categorical sense, as the functor is covariant, not contravariant.) Cellular algebras are quasihereditary provided a "non-degeneracy" property is satisfied [29]; this fails for the TL algebra in the case $m=0$ because, as mentioned above, there are less than $L+1$ non-zero simple modules. For our purposes, the full strength of this theory is not needed, and for the most part our arguments go through in the case $m=0$ as well as $m \neq 0$.

\section{Morita equivalence as associative algebras}

Now we consider the commutant of TL in the module $V$ again, from a general point of view. For commutants, we will follow the useful convention that the commutant of an algebra that acts on a module on the right is viewed as an algebra acting on the same module on the left, and vice versa. Here we will view $V=V_{\mathrm{TL}}$ as a right module over the TL algebra. Thus we can write $V={ }_{\mathcal{A}} V_{\mathrm{TL}}$ to record this fact; $V$ is thus a left $\mathcal{A}$-, right TL-, bimodule (we let TL stand for the $\mathrm{TL}_{2 L}(q)$ and $\mathcal{A}$ for $\mathcal{A}_{m+n \mid n}(2 L)$ ). We will need the following useful Theorem [52]: Let $R$ be an algebra, $M$ a finitely-generated right module over $R$, and $S$ the commutant of $R$ in $M$. Then there is an equivalence between the category of direct summands (as right $R$-modules) in direct sums of copies of $M$, and the category of projective right modules over $S$ (and similarly with "right" replaced by "left" everywhere). For finitedimensional $R$ and $M$, we may think of the objects in the former category more simply as direct sums of the summands in $M$. The equivalence is constructed using the module $M$ itself [52], as we will describe momentarily.

By taking $\mathrm{TL}_{2 L}(q)$ as $R$ and using either our spaces $V$, or the spin- $1 / 2$ chain, for $M$, and the respective commutants as $S$, we obtain category equivalences between the direct summands as right TL modules, and the projective right modules over $S$. We again denote the commutant algebra of $\mathrm{TL}_{2 L}(q)$ in $V$ by $\mathcal{A}_{m+n \mid n}(2 L)$, and that in the spin- $1 / 2$ chain by $U_{q}\left(\mathrm{sl}_{2}\right)^{(2 L)}$. (We should mention that when $q$ is a root of unity, $U_{q}\left(\mathrm{sl}_{2}\right)^{(2 L)}$ is an image of the version of $U_{q}\left(\mathrm{sl}_{2}\right)$ called the "restricted specialization" in Ref. 25], which includes the so-called renormalized powers of the generators that can be defined by a limiting process as $q$ tends to the root of unity through the complex numbers.) The TL algebra acts faithfully in both cases, and provided that the direct summands under TL present in one chain are isomorphic to direct summands present in the other, then for each $m$ and $L$ we obtain equivalences between the categories of projective right modules over $\mathcal{A}_{m+n \mid n}(2 L)$ (for all $n \geq 0$ such that $m+2 n>1)$, and over $U_{q}\left(\mathrm{sl}_{2}\right)^{(2 L)}$ for $q$ corresponding to $m$.

For the spin- $1 / 2$ chain, the TL algebra and the decomposition of the chain have been much studied [53, 54, 55, 56]. For our chains with TL acting in the space $V$, we can apply an argument similar to that in Sec. 4.1 of Ref. [56], to show that the direct sum decomposition is the same as in the spin- $1 / 2$ chain, with non-zero multiplicities of all summands provided $m+2 n>1$. A sketch of the argument goes as follows; it uses some of the concepts of the preceding subsection. First we construct a set of states for each $j$ as follows. We take each valid pattern as in Sec. II, with $2 j$ dots, and as before form a corresponding state of $V$ by forming a singlet for each contracted pair of sites, and placing states on the dots that are annihilated by $e_{i}$ acting on these dots as if the contracted pairs were absent. The cardinality of this set is then $d_{j} D_{j}^{\prime}$, but we have not shown that the vectors are linearly independent, so the dimension of the subspace $\mathcal{K}_{j}$ they span could be less. However, for each fixed state on the dots, which is an element of the $j$ th standard module over the commutant $\mathcal{A}$, the set of valid patterns forms the standard module of TL. That is, there exists an injective homomorphism of the $j$ th standard module of TL into $\mathcal{K}_{j}$. Similarly, for any fixed valid pattern, the states on the dots form the $j$ th standard module of the commutant $\mathcal{A}$, so there is an injective homomorphism of the $j$ th standard module of $\mathcal{A}$ into $\mathcal{K}_{j}$. For some $q$ and some $j$, the $j$ th standard module of TL is simple, and then $\mathcal{K}_{j}$ has dimension $d_{j} D_{j}^{\prime}$. But when, as mentioned in the previous subsection, the standard module of TL contains a simple submodule (with simple quotient module), $\mathcal{K}_{j}$ may decompose as a TL module into a non-zero number of full standard modules, and some number of simple quotient modules. If one takes the quotient by all the remaining submodules, the number of simple quotients is $D_{j}^{\prime}$. One further point is that the subspaces $\mathcal{K}_{j}$ for different $j$ are not necessarily linearly independent of each other, but may "overlap", where this is allowed by the 
representation theory of TL. That is, some of the copies of simple quotient modules may coincide with the submodules in standard modules in one of $\mathcal{K}_{j}$ at a smaller $j$. It is also true that as a TL module, $V$ is self-dual under the natural duality of left TL modules to left TL modules mentioned above. It must decompose as a sum of indecomposable TL modules that are either self-dual, or come in dual pairs. Now our task is to find a faithful TL module that contains submodules isomorphic to $\mathcal{K}_{j}$ for all $j$, is self-dual, and that has the same dimension as $V$, namely $(m+2 n)^{2 L}=\sum_{j=0}^{L} d_{j} D_{j}^{\prime}$. These requirements are highly restrictive. Using the known representation theory of the TL algebra [22], the structure (decomposition) of such a module is uniquely fixed, and is the same as that of the spin- $1 / 2$ chain (except that the multiplicities are different, though still nonzero); this then must be the structure of $V$. It turns out that all indecomposable summands are self-dual, and that there is the maximum possible overlap of the spaces constructed above. The direct sum decomposition is described for moderate $L$ in Ref. [56], and we have checked in several cases that we find the same in $V$. We give further details in the cases of physical interest, $m=0, \pm 1$, in another paper [13].

The resulting equivalences of categories can be described explicitly as follows [52] [the structure is the same if our chain $V$ is replaced by the spin-1/2 chain, and $\mathcal{A}$ by $\left.U_{q}\left(\mathrm{sl}_{2}\right)\right]$. The functor from right $\mathcal{A}$ modules to right TL modules maps any right $\mathcal{A}$ module $\mathcal{V}$ to the tensor product, $\mathcal{V} \otimes_{\mathcal{A}} V$, which is a right TL-module. (Here we use the general tensor product over a non-commutative ring $R$ : if $M_{R}$ is a right $R$-module, and ${ }_{R} N$ is a left $R$-module, then $M \otimes_{R} N$ is the tensor product, which is the usual space spanned by bilinears modulo the relations $m \otimes a n=m a \otimes n$ for all $m \in M, n \in N, a \in R$. If $R$ is the complex numbers, we write simply $\otimes$ as before.) The functor in the reverse direction is given on any right TL-module $\mathcal{W}$ by $\operatorname{Hom}_{\mathrm{TL}}\left({ }_{\mathcal{A}} V_{\mathrm{TL}}, \mathcal{W}\right)$, which is a right $\mathcal{A}$ module. (Here we use the $\operatorname{Hom}_{R}\left(M_{R}, N_{R}\right)$ space, the vector space of $R$-homomorphisms between right $R$-modules $M_{R}, N_{R}$. If $M_{R}$ is also a left $S$-module, $M={ }_{S} M_{R}$, then the $\operatorname{Hom}_{R}$ space is naturally a right $S$ module. When $R$ is the complex numbers, it will be denoted simply Hom.) Both maps are functors which also define maps of morphisms to morphisms in the respective categories of modules. When these functors are restricted to the subcategories of summands in direct sums of copies of $V$ (as TL modules), and projective modules over $\mathcal{A}$, the equivalence of these latter categories is obtained [52].

As the algebras are finite-dimensional, the resulting equivalence of the categories of projective right modules over $\mathcal{A}$ and over $U_{q}\left(\mathrm{sl}_{2}\right)$ can be extended to an equivalence of the categories of all right, and of all left, modules, that is for each $m$, a Morita equivalence between $\mathcal{A}_{m+n \mid n}(2 L)$ (for all $n \geq 0$ such that $m+2 n>1$ ) and $U_{q}\left(\mathrm{sl}_{2}\right)^{(2 L)}$. [These Morita equivalences are given by similar functors as those described above, but with a suitable $\mathcal{A}$-TL bimodule (a projective generator) in place of $V$ [19].] Put more simply, one can read off the structure of the commutant algebra of TL in the module $V$, or in the spin-1/2 chain, from the structure of the chain as a TL module. One only has to find all endomorphisms of the TL algebra in the given module; these form the commutant. The structures of these commutants are the same (though the multiplicities differ), so the algebras are Morita equivalent.

In the non-semisimple cases, this argument does not establish Morita equivalence of the TL algebra and its commutant, which does not always hold, or else is not necessarily of the same form as in the semisimple cases: for example, when $m=0$ the number of simple modules in the two algebras is different for all $L$. For $|m|<2$, $m \neq 0$, it appears that there is a Morita equivalence between TL and its commutant, but the correspondence is obtained by reversing the ordering (analogous to our labels $j$ in the semisimple cases) of the modules within each indecomposable block of the algebras [56]. This equivalence does not appear to be useful for our purposes.

We also find that the commutant of $\mathcal{A}_{m+n \mid n}(2 L)$ in $V$ is just the TL algebra (not larger), so they form a "dual pair", as is also the case for $U_{q}\left(\mathrm{sl}_{2}\right)^{(2 L)}$ in the spin-1/2 chain [56]. In the $U_{q}\left(\mathrm{sl}_{2}\right)$ case, this assertion is known as quantum Schur-Weyl reciprocity [49, 50]. Then we can apply the Theorem with $\mathcal{A}_{m+n \mid n}(2 L)$ as $R$ and $\mathrm{TL}_{2 L}(q)$ as $S$, with $V$ as a left $\mathcal{A}$-module. In the case of the spin- $1 / 2$ chain, the decomposition into a direct sum under $U_{q}\left(\mathrm{sl}_{2}\right)$ was studied in Ref. [36]. The direct summands are a certain type of $U_{q}\left(\mathrm{sl}_{2}\right)$ module, which (together with analogs for other quantized UEAs) are now known to be examples of "tilting modules" [25]. More precisely, there is another, more general definition of tilting modules [25], and the spin-1/2 chain is a "full tilting module", which means it is tilting, and that its direct sum decomposition into indecomposable tilting modules contains at least one copy of each indecomposable tilting module over $U_{q}\left(\mathrm{sl}_{2}\right)^{(2 L)}$ - except for $m=0$, when the $j=0$ singlet tilting module is not a summand in the chain, [and the same is true for its Morita equivalent module over $\left.\mathcal{A}_{m+n \mid n}(2 L)\right]$. As the same (complete) set of indecomposable projective left modules for the TL algebra arises in connection with both $V$ and the spin- $1 / 2$ chain, it follows that the category of left tilting modules for $U_{q}\left(\mathrm{sl}_{2}\right)^{(2 L)}$ and its analog for $\mathcal{A}_{m+n \mid n}(2 L)$ are equivalent (and this equivalence is the same as the restriction of the previous one to the tilting modules). We will call these summand modules for $\mathcal{A}_{m+n \mid n}$ "tilting modules" also. We have also checked explicitly for some cases that the modules of the commutant in $V$ have the same submodule structure as those of $U_{q}\left(\mathrm{sl}_{2}\right)$ in the spin-1/2 chain [36, 56]. We will term the direct summands of the TL algebra in $V$ "tilting modules" (of TL) also.

The tilting modules, viewed as direct summands in $V$ as an $\mathcal{A}$-module, are related to the standard modules. Tilting modules that are simple are standard, while tilting modules that are not simple can be decomposed into a sub-module and a quotient module that are both standard [25, 36, 50]. Tilting modules are generally defined 
as having such a series decomposition, and also one for their dual. In the present cases, all tilting modules are self-dual (when duality is viewed as a map from left modules to left modules).

\section{D. $L \rightarrow \infty$ limit}

Next we discuss the $L \rightarrow \infty$ limits of the algebras, their modules, and of the equivalence between them. Again, this requires somewhat more care in the non-semisimple cases. There are two different limits that can be taken for the TL algebra and for its commutant. Just one of these is a limit of TL as an algebra, while the other is a limit of the commutant algebras $\mathcal{A}$ or $U_{q}\left(\mathrm{sl}_{2}\right)$, but both also give rise to limits of the categories of modules on both sides, that is TL and its commutant. The more useful one of these two limits seems to be the one that arises from the natural quotient (projection) maps of cellular algebras on the commutant side. This emphasizes the usefulness of the symmetry analysis, and we will learn more about this in the following subsection when we consider the product operations.

For the TL algebras, $\mathrm{TL}_{2 L}(q)$ is a subalgebra of $\mathrm{TL}_{2 L+2}(q)$. It can be defined as an injection of the former into the latter, in an obvious way that takes $e_{i}$ to $e_{i}$ for $i=0, \ldots, 2 L-2$, and the unit 1 to 1 . This map from $L \rightarrow L+1$ can be iterated, and one obtains a compatible system of algebra homomorphisms from $\mathrm{TL}_{2 L_{1}}(q)$ to $\mathrm{TL}_{2 L_{2}}(q)$ for all $L_{2} \geq L_{1}$. By a standard construction (see, e.g., Ref. [57]), one obtains from this the purely algebraic "direct" (or "inductive") limit of the algebra (the $C^{*}$-algebra version of this was used extensively for semisimple quotients of TL in Refs. [20, 21]). The direct limit algebra is generated by 1 and $e_{i}, i=0,1$, $2, \ldots$, subject to the relations (6), and elements of the limit algebra are finite linear combinations of products of a finite number of $e_{i} \mathrm{~s}$, and thus every element lies in $\mathrm{TL}_{2 L}(q)$ for some $L$. Notice that the left end of the chain is held fixed in the limit, while the right end goes to infinity. One could also define the direct limit in other ways, for example holding the right end of the chain fixed, with generators $e_{i}$, with $i=\ldots,-1,0$, by subtracting $2 L-2$ from $i$ [for $\mathrm{TL}_{2 L}(q)$ ] before defining the injection $\mathrm{TL}_{2 L}(q) \rightarrow \mathrm{TL}_{2 L+2}(q)$ by $e_{i} \rightarrow e_{i}$. Other ways include letting both ends go to infinity. Thus there is more than one way to define such a limit, because it depends on the choice of the system of injections. We will nonetheless continue the discussion a little, using the former definition of the direct limit.

Given a choice of injections $\mathrm{TL}_{2 L}(q) \rightarrow \mathrm{TL}_{2 L+2}(q)$ for all $L$, we can use the induction functor to map modules of the former to modules of the latter (this functor may be familiar from the operation of inducing a representation of a group from a representation of a subgroup). The induction functor is defined as follows. If an algebra $R$ is a subalgebra of $T$, then $T$ itself can be naturally viewed as left module over itself, and as a right module over $R$; this is written as $T_{T} T_{R}$. If $M$ is a left $R$ module, then the induced left $T$-module can be obtained as ${ }_{T} T_{R} \otimes_{R} M$. This map from $L \rightarrow L+1$ can be iterated, and one obtains a compatible system of functors from modules over $\mathrm{TL}_{2 L_{1}}(q)$ to modules over $\mathrm{TL}_{2 L_{2}}(q)$ for all $L_{2} \geq L_{1}$. The direct (or inductive) limit of this family can then be taken. It can be shown that the induction functor maps projective modules of $\mathrm{TL}_{2 L_{1}}(q)$ to projectives of $\mathrm{TL}_{2 L_{2}}(q)$. Then by composing with the equivalence with the categories of direct summands (tilting modules) of the commutants, we also obtain a functor from the category of tilting modules of $\mathcal{A}_{m+n \mid n}\left(2 L_{1}\right)$ to that of $\mathcal{A}_{m+n \mid n}\left(2 L_{2}\right)$. This functor can be described directly. From $\mathcal{A}_{m+n \mid n}(2 L)$ one can form the Morita equivalent algebra $\mathcal{A}_{m+n \mid n}\left(2 L_{1}\right) \otimes M_{(m+2 n)^{2}}(\mathbf{C})$, as follows. All modules of the latter are obtained by tensoring a module of the former with $\mathbf{C}^{(m+2 n)^{2}}$, which describes two additional sites of the chain, and this defines the Morita equivalence. $\mathcal{A}_{m+n \mid n}(2 L+2)$ is a subalgebra of this larger algebra. Then one can apply the functor of restriction of modules to obtain a module over $\mathcal{A}_{m+n \mid n}(2 L+2)$ from a module over $\mathcal{A}_{m+n \mid n}\left(2 L_{1}\right) \otimes M_{(m+2 n)^{2}}(\mathbf{C})$, and we have seen that the latter are generated by tensor product modules. As restriction maps direct summands to direct summands, we are done.

We have found a system of functors mapping modules over the commutant for smaller to those for larger $L$, and we might expect that there is a corresponding injection of the smaller algebra into the larger. For the semisimple cases, there are injection maps, but not for the nonsemisimple cases. In no cases are there injection maps of algebras that agree with the functors defined above on the modules.

On the hand, as we have already seen, there is a compatible family of quotient (or projection) homomorphisms from the commutant $\mathcal{A}_{m+n \mid n}\left(2 L_{2}\right)$ to $\mathcal{A}_{m+n \mid n}\left(2 L_{1}\right)$ for $L_{2}>L_{1}$, which can be obtained by iterating the maps $p_{2 L+2}: \mathcal{A}_{m+n \mid n}(2 L+2) \rightarrow \mathcal{A}_{m+n \mid n}(2 L)$ that we have already described; the kernel of the map $p_{2 L+2}$ is the cellular ideal $\mathcal{A}^{(2 L+2)}$ in $\mathcal{A}_{m+n \mid n}(2 L+2)$. From this we can then define the limit algebra $\mathcal{A}_{m+n \mid n}$ as the "inverse" (or "projective") limit of the system of projection (quotient) maps (see, e.g., Ref. [58]). In effect, this means that the limit algebra $\mathcal{A}_{m+n \mid n}=\mathcal{A}$ is cellular with an infinite descending chain of ideals

$$
\cdots \subset \mathcal{A}^{(4)} \subset \mathcal{A}^{(2)} \subset \mathcal{A}^{(0)}=\mathcal{A} .
$$

(Thus the limit algebra is not artinian, though it is still noetherian.) As the finite-dimensional quotient algebras $\mathcal{A}(2 L) / \mathcal{A}^{(k)}(2 L)$ for each $k(k \leq 2 L)$ are isomorphic for all $L$, this limit seems extremely natural and corresponds under Morita equivalence to the inverse limit $U_{q}\left(\mathrm{sl}_{2}\right)$ of the finite-dimensional algebras. That is, we will define $U_{q}\left(\mathrm{sl}_{2}\right)$ itself as the inverse limit of the $q$-Schur algebras $U_{q}\left(\mathrm{sl}_{2}\right)^{(2 L)}$, and this limit is Morita equivalent to our algebras $\mathcal{A}_{m+n \mid n}$. As usual, for the oriented-loops models we mean here the quotient algebra of $U_{q}\left(\mathrm{sl}_{2}\right)$ with integer spin $(j)$ representations only. 
Again, there are closely related functors on the categories of modules over $\mathcal{A}_{m+n \mid n}(2 L)$. Given the projection maps $p_{2 L+2}$ for the algebras, there are natural "pullback" functors which "lift" any module over $\mathcal{A}_{m+n \mid n}(2 L)$ to a module over $\mathcal{A}_{m+n \mid n}(2 L+2)$. The lifted module is the same as the original when viewed as a (graded) vector space, so this functor preserves dimensions, unlike the induction functor which was applied on the TL side above. The system of compatible pullback functors itself has a direct limit, and the limit object is a category of modules over the limit algebra $\mathcal{A}_{m+n \mid n}$, such that any module over any of the $\mathcal{A}_{m+n \mid n}(2 L)$ 's is mapped to a module over the limit algebra $\mathcal{A}_{m+n \mid n}$, functorially. A natural category of such modules over the limit algebra to consider is that in which every module is the lift of a module over $\mathcal{A}_{m+n \mid n}(2 L)$ for some $L$; this includes the lifts of all the finite-dimensional modules over $\mathcal{A}_{m+n \mid n}(2 L)$ for all $L$, and is the smallest possible direct limit category. Again, one can define a similar functor on, and category of, $U_{q}\left(\mathrm{sl}_{2}\right)$ modules, and Morita equivalence is preserved in the limit. The pullback functors map tilting modules to tilting modules, and also standard modules to standard modules, in particular mapping the $j$ th indecomposable of either type to the corresponding one for $L \rightarrow L+1$. It is then natural to speak of tilting and standard modules for the limit algebra, as well as for finite $L$.

In addition, the functor that yields an equivalence of the categories of tilting modules for $\mathcal{A}(2 L)$ [or for $\left.U_{q}\left(\mathrm{sl}_{2}\right)^{(2 L)}\right]$ to that of projective modules over TL can be composed with those above to produce functors from the category of modules over $\mathrm{TL}_{2 L}(q)$ to that for $\mathrm{TL}_{2 L+2}(q)$, which are not the same as the induction functors constructed above. The present functors map the $j$ th indecomposable projective module for $\mathrm{TL}_{2 L}(q)$ to the corresponding (i.e., $j$ th one) for $\mathrm{TL}_{2 L+2}(q)$, unlike the induction functors above which act in a more complicated way. We can take the smallest direct limit of this compatible family of functors also. The result is a category all of whose objects are infinite dimensional vector spaces; every object is obtained by applying the composite of the infinite sequence of functors to a module in the category of modules for $\mathrm{TL}_{2 L}(q)$ for some size $L$, and similarly for the morphisms. By using the alternative definition of projective modules that is expressed entirely in terms of morphisms and objects [19], it follows that the limit of a projective module taken in this way is a "projective object" in the limit category. Because the projection maps of the commutant algebras, and the functors that follow from them, are defined in an essentially unique way, this construction is not plagued by non-uniqueness issues as the induction on the TL side was. These reasons of simplicity and uniqueness are what make the present way of taking the limit seem the most natural one for our purposes, and we will see more on this in the next two subsections. Note that there are no projection homomorphisms for the TL algebras taking $L+1$ to $L$, even in the semisimple cases $|m| \geq 2$, as can be seen by considering the effect they would have on dimensions of modules as vector spaces. While the direct limit of the functors exists as a category, it is not clear to us whether there is a corresponding (purely algebraic) limit of the TL algebras as well, for which this would be a category of modules (but see also Sec. VIII below).

\section{E. Tensor products and ribbon Hopf structure}

In this subsection we consider the additional structures that turn the commutant algebras $\mathcal{A}_{m+n \mid n}$ into ribbon Hopf algebras, and show that these are Morita equivalent as ribbon Hopf algebras to $U_{q}\left(\mathrm{sl}_{2}\right)$. The most important of these structures is the comultiplication (or tensor product of modules).

A comultiplication for the commutant can be defined naturally. First, a chain of $2 L=2\left(L_{1}+L_{2}\right)$ sites can be broken into two of lengths $2 L_{1}, 2 L_{2}$ by removing the generator $e_{2 L_{1}-1}$ that connects them. This shows that $\mathrm{TL}_{2 L_{1}} \otimes \mathrm{TL}_{2 L_{2}}$ is (isomorphic to) a subalgebra of $\mathrm{TL}_{2 L}$ for $L=L_{1}+L_{2}$ (and the same value of $q$ throughout). Then, taking the commutants of these algebras in the chain $V$ we see that $\mathcal{A}_{m+n \mid n}(2 L)$ is a subalgebra of $\mathcal{A}_{m+n \mid n}\left(2 L_{1}\right) \otimes \mathcal{A}_{m+n \mid n}\left(2 L_{2}\right)$. We define the natural inclusion (or injection) map $\mathcal{A}_{m+n \mid n}(2 L) \rightarrow$ $\mathcal{A}_{m+n \mid n}\left(2 L_{1}\right) \otimes \mathcal{A}_{m+n \mid n}\left(2 L_{2}\right)$ to be $\Delta_{2 L_{1}, 2 L_{2}}$. In order to take the inverse limits of these algebras and so obtain an inclusion $\Delta: \mathcal{A}_{m+n \mid n} \rightarrow \mathcal{A}_{m+n \mid n} \otimes \mathcal{A}_{m+n \mid n}$, we should show that the inclusions for finite length are compatible with the projection maps $p_{2 L}$ (etc.). That is, we should prove that for all $L_{1}, L_{2}\left(L_{1}+L_{2}=L\right), \Delta_{2 L_{1}, 2 L_{2}} \circ p_{2 L+2}=$ (id $\left.\otimes p_{2 L_{2}+2}\right) \circ \Delta_{2 L_{1}, 2 L_{2}+2}$ (and similarly for increasing $\left.L_{1} \rightarrow L_{1}+1\right)$, so that the double inverse limit $\Delta$ of $\Delta_{2 L_{1}, 2 L_{2}}$, as $L_{1}, L_{2} \rightarrow \infty$, exists. This is fairly straightforward, and we note only that $\operatorname{ker} \Delta_{2 L_{1}, 2 L_{2}} \circ p_{2 L+2}=$ $\operatorname{ker}\left(\mathrm{id} \otimes p_{2 L_{2}+2}\right) \circ \Delta_{2 L_{1}, 2 L_{2}+2}=\mathcal{A}_{m+n \mid n}^{(2 L+2)}(2 L+2)$ for all $L_{1}, L_{2}$. Then $\Delta$ is also co-associative, and is the required comultiplication $\Delta: \mathcal{A}_{m+n \mid n} \rightarrow \mathcal{A}_{m+n \mid n} \otimes \mathcal{A}_{m+n \mid n}$. In terms of the elements $J_{b_{1} \cdots b_{k}}^{a_{1} \cdots a_{k}}$, the comultiplication acts in exactly the same way as in the semisimple case (except again that the use of the Jones-Wenzl projectors should be avoided).

The main use of the comultiplication is its application to modules. The pullback of the injection map $\Delta_{2 L_{1}, 2 L_{2}}$ maps a module over $\mathcal{A}_{m+n \mid n}\left(2 L_{1}\right) \otimes \mathcal{A}_{m+n \mid n}\left(2 L_{2}\right)$ to one over $\mathcal{A}_{m+n \mid n}(2 L)$. In particular, a tensor product of a module over $\mathcal{A}_{m+n \mid n}\left(2 L_{1}\right)$ and one over $\mathcal{A}_{m+n \mid n}\left(2 L_{2}\right)$ is naturally a module over $\mathcal{A}_{m+n \mid n}\left(2 L_{1}\right) \otimes \mathcal{A}_{m+n \mid n}\left(2 L_{2}\right)$. The pullback functor in the case when the algebra homomorphism is an injection is more often called "restriction" (it is the "right adjoint" to the induction functor for the same map [19]). It can be written explicitly as the tensor product with

$$
\mathcal{A}_{m+n \mid n}(2 L) \mathcal{A}_{m+n \mid n}(2 L)_{\mathcal{A}_{m+n \mid n}\left(2 L_{1}\right) \otimes \mathcal{A}_{m+n \mid n}\left(2 L_{2}\right)},
$$

which is the algebra $\mathcal{A}_{m+n \mid n}(2 L)$ viewed as a left 
$\mathcal{A}_{m+n \mid n}(2 L)$-, right $\mathcal{A}_{m+n \mid n}\left(2 L_{1}\right) \otimes \mathcal{A}_{m+n \mid n}\left(2 L_{2}\right)^{-}$, bimodule. As the tensor product (over $\mathcal{A}$ ) is distributive over direct sums, the restriction functor, and thus the product operation automatically maps a product of tilting modules to a tilting module (this argument requires some modification to handle the case of the $j=0$ tilting module for $m=0$, which is not a direct summand in the chain; however, this is easily done directly).

To obtain a similar restriction map for the limit, we may note that the equal composite maps $\Delta_{2 L_{1}, 2 L_{2}} \circ$ $p_{2 L+2}=\left(\mathrm{id} \otimes p_{2 L_{2}+2}\right) \circ \Delta_{2 L_{1}, 2 L_{2}+2}$ have equal pullback functors, which are the composition of that taking modules for $L$ to one for $L+1$, as discussed in the preceding section, with the pullback of $\Delta_{2 L_{1}, 2 L_{2}}$ or $\Delta_{2 L_{1}, 2 L_{2}+2}$. Thus the result of restricting using $\Delta_{2 L_{1}, 2 L_{2}}$ (or $\Delta_{2 L_{1}, 2 L_{2}+2}$ ) is the same either before or after applying the appropriate lift maps for $L \rightarrow L+1$ (or $L_{1} \rightarrow L_{1}$, $\left.L_{2} \rightarrow L_{2}+1\right)$. Hence the limit of the restriction maps exists, and we obtain a tensor product operation from the category of modules over $\mathcal{A}_{m+n \mid n}$ to itself, that is it makes the product module over $\mathcal{A}_{m+n \mid n} \otimes \mathcal{A}_{m+n \mid n}$ into one over $\mathcal{A}_{m+n \mid n}$, and further this tensor product is also associative. Thus the category of modules over $\mathcal{A}_{m+n \mid n}$ is now a tensor category, as is the subcategory of tilting modules (as for $U_{q}\left(\mathrm{sl}_{2}\right)$ [36]). The fusion rules for this product in non-semisimple examples will be discussed in Ref. [13], but we note that the one-dimensional "singlet" module, which is tilting, is the unit for the tensor product. The unit and co-unit maps for the algebra $\mathcal{A}_{m+n \mid n}$ exist, the co-unit $\varepsilon$ being the map to the complex numbers, defined as the limit of the projection maps $\mathcal{A}_{m+n \mid n}(2 L) \rightarrow \mathcal{A}_{m+n \mid n}(2 L) / \mathcal{A}_{m+n \mid n}^{(2)}(2 L) \cong \mathbf{C}$. The counit map is used to show that the singlet module is the unit for the tensor product of modules [25, 26]. The unit map $\eta$ simply maps a complex number $c$ to $c .1$ in $\mathcal{A}$.

To understand the antipode, we will begin by considering natural operations on the space $V$ for $2 L$ sites, as for the other structures. The antipode is used to turn the left dual vector space $\mathcal{V}^{*}$ of any module $\mathcal{V}$ into a left module. In particular, we can consider the module $V$, and infer the result. If we consider the birth maps discussed in the Appendix, and apply to the case of $V$, we will obtain a map $b_{V}: \mathbf{C} \rightarrow V \otimes V^{*}$. We can identify $V^{*}$ with $V$ as before, and then we require that the image of $b_{V}$ in $V \otimes V$ be invariant under $\mathcal{A}$, that is the action of any $a \in \mathcal{A}$ on this image is simply given by multiplication by $\varepsilon(a)$ (this means that it is annihilated by all ideals $J^{(k)}$ for $k>0$; it is isomorphic to the singlet or $j=0$ standard module). The image of this map is simply described as the state corresponding to the pattern (of singlet contractions of $V_{0}$ with $V_{0}^{*}$ in one or the other order) $((\cdots))$ of $2 L$ left and $2 L$ right parentheses. Now we consider the action of $\mathcal{A}$ on this module; this requires the use of the comultiplication, while the action of $\mathcal{A}$ on each tensor factor is known because $\mathcal{A}$ was defined as an algebra of endomorphisms on such modules. From the definition of the comultiplication $\Delta$, the action of $\mathcal{A}$ on $V(2 L) \otimes V(2 L)$ is identical to that on $V(4 L)$. The image of $b_{V}$ is clearly in the singlet subspace, but it may be useful to prove this here as the corresponding proof was not given elsewhere in this paper. We consider one of the operators $\hat{j}$ defined in the Appendix, that is a linear combination of the operators $\widetilde{J}$ containing $k$ factors of $J_{i a}^{b}$. Consider also the inner-most parentheses, the pair (). In the sum over positions $i_{l}$, there are terms with zero, one or two operators $j_{i}$ on these two sites [which are $2 L-1,2 L$ in the labeling of $V(4 L)$.] The terms with one or two operators at these sites annihilate this singlet, as in the construction of $\hat{j}$. That leaves terms in which no operators are on those sites, and that pair can effectively be deleted from the problem. Then arguing by induction completes the proof. There are similar proofs that operators with $k>2 j$ annihilate the states described by valid patterns with $2 j$ non-contractible dots, which transform as the $j$ th standard module. Similarly, one can see directly that the ideal $T^{(2 j)}$ is annihilated by the operators $\widehat{j}$ with $k>2 j$.

This calculation shows that the left dual $V^{*}$ of $V$ is isomorphic to $V$ as a left $\mathcal{A}$-module. The action of $a \in \mathcal{A}$ on an element $w$ in the dual module $V^{*}$ is supposed to be given by $S(a)^{*} w$, where $S$ is the antipode. On using the isomorphism from $V^{*}$ to $V$, this becomes $S(a)^{s} v$ for the image $v \in V$ of $w$, where ${ }^{s}$ is the anti-involution already discussed. But $V$ is the defining representation for $\mathcal{A}$, and so the $\mathcal{A}$ action is also given by av. Taking matrix elements, $S(a)^{s}=a$ for all $a \in \mathcal{A}$, and so (as ${ }^{s}$ is an anti-involution), $S(a)=a^{s}$. The explicit action of ${ }^{s}$ is discussed further in the Appendix, and then to the extent that we may use the notation $J_{b_{1} \ldots b_{k}}^{a_{1} \ldots a_{k}}, S$ is given by

$$
\begin{aligned}
S\left(J_{b_{1} \ldots b_{k}}^{a_{1} \ldots a_{k}}\right)= & (-1)^{k}(-1)^{\sum_{l<l^{\prime}}\left(\operatorname{deg} a_{l}+\operatorname{deg} b_{l}\right)\left(\operatorname{deg} a_{l^{\prime}}+\operatorname{deg} b_{l^{\prime}}\right)} \\
& \times J_{b_{k} \ldots b_{1}}^{a_{k} \ldots a_{1}} .
\end{aligned}
$$

Clearly this agrees with the cases $n=0$ discussed earlier; it is the general formula for the cases $|m| \geq 2$ as well. This construction of $S$ on the algebras for finite length chains passes immediately to the $L \rightarrow \infty$ limit. We note that the algebra $\mathcal{A}$ in these oriented-loops cases is spanned by the elements in the $J^{(k)} \mathrm{s}$ for $k$ even, so for these the sign $(-1)^{k}$ can be dropped.

These make $\mathcal{A}_{m+n \mid n}$ into a Hopf algebra, which is Morita equivalent to $U_{q}\left(\mathrm{sl}_{2}\right)$ as a Hopf algebra. The braiding and twist can be introduced using the TL generators exactly as in the semisimple cases, and the Morita equivalence now extends to the algebras as ribbon Hopf algebras. As a Hopf algebra, $\mathcal{A}$ is an extension of $U(\operatorname{gl}(m+n \mid n)$, but the braiding and twist are not extensions of those that make $U(\operatorname{gl}(m+n \mid n)$ a ribbon Hopf algebra. We note further that the unoriented cases carry through in an exactly parallel way to the oriented and semisimple unoriented ones.

We have concentrated here on the faithful cases $m+$ $2 n \geq 2$. It is worth noting that for $m=0, n=1$, the supersymmetric chain has a formulation as a free (unconstrained) fermion system [8]; see the Appendix. For 
this case $D_{j}^{\prime}=2 j+1$. In fact, the commutant algebras $\mathcal{A}_{1 \mid 1}(2 L)$ and $U_{q}\left(\mathrm{sl}_{2}\right)^{(2 L)}$ with $q=i$ are isomorphic (and not only Morita equivalent) as associative algebras for each $L$, and the limits $\mathcal{A}_{1 \mid 1}$ and $U_{q}\left(\mathrm{sl}_{2}\right)$ are isomorphic [59] as ribbon Hopf algebras (the difference between the graded tensor products used for $\mathcal{A}_{1 \mid 1}$, and the trivially graded ones for $U_{q}\left(\mathrm{sl}_{2}\right)$, is absorbed into the maps of the comultiplication and antipode under this isomorphism).

\section{F. Induction product for TL modules}

There is also a product operation for the TL modules, which is the analog of the tensor product of modules over the commutant, and is again defined using the picture of joining chains end to end. It has to be defined using the induction functor, and does not preserve dimensions of modules. Here we define it, and show that the fusion rules for the product of two projective modules over TL agree with those for the corresponding tilting (summand) modules over the commutant $\mathcal{A}$ or $U_{q}\left(\mathrm{sl}_{2}\right)$.

For TL one uses again the inclusion of $\mathrm{TL}_{2 L_{1}} \otimes \mathrm{TL}_{2 L_{2}}$ (in an obvious way) as a subalgebra of $\mathrm{TL}_{2 L}$ for $L=$ $L_{1}+L_{2}$. Then given any left TL-module $\mathcal{W}$ over $\mathrm{TL}_{2 L_{1}} \otimes \mathrm{TL}_{2 L_{2}}$, one can apply the induction functor to obtain a module over $\mathrm{TL}_{2 L}$, given explicitly by

$$
\mathrm{TL}_{2 L} \otimes_{\mathrm{TL}_{2 L_{1}} \otimes \mathrm{TL}_{2 L_{2}}} \mathcal{W}
$$

where the algebra $\mathrm{TL}_{2 L}$ is viewed as a left $\mathrm{TL}_{2 L}$, right $\mathrm{TL}_{2 L_{1}} \otimes \mathrm{TL}_{2 L_{2}}$ module (it is a right $\mathrm{TL}_{2 L_{1}} \otimes \mathrm{TL}_{2 L_{2}}$ by restriction). It is a general fact that any induction functor maps projective modules to projective modules, so in particular, when applied to a projective module over $\mathrm{TL}_{2 L_{1}} \otimes \mathrm{TL}_{2 L_{2}}$, such as a tensor product of a projective of $\mathrm{TL}_{2 L_{1}}$ with a projective of $\mathrm{TL}_{2 L_{2}}$, the result of induction is a projective module over $\mathrm{TL}_{2 L}$. Notice that, like induction in general, this product operation does not conserve the dimensions of the modules.

Given this product operation (functor), which maps a tensor product of projective modules for lengths $2 L_{1}$, $2 L_{2}$, to a projective module for length $2 L$, it is natural to ask how it compares with the product operation that was defined for modules over the commutant, and in particular for the product of tilting modules, since these correspond to the projective modules over TL under the equivalence of categories. We can utilize the functor from left TL modules to left modules over the commutant either before or after taking the product. The functor for the length $2 L$ chain is the tensor product functor

$$
\mathcal{A}_{m+n \mid n}(2 L) V_{\mathrm{TL}_{2 L}(q)} \otimes_{\mathrm{TL}_{2 L}(q)}-
$$

and similarly for $\mathrm{TL}_{2 L_{1}} \otimes \mathrm{TL}_{2 L_{2}}$. Thus all the functors involved can be written as tensor products with suitable modules, either $V$ or one of the algebras. Using associativity of the tensor product over non-commutative algebras, we find that both orders of functor operations reduce to the tensor functor

$$
\mathcal{A}_{m+n \mid n}(2 L) V_{\mathrm{TL}_{2 L_{1}}(q) \otimes \mathrm{TL}_{2 L_{2}}(q)} \otimes_{\mathrm{TL}_{2 L_{1}}(q) \otimes \mathrm{TL}_{2 L_{2}}(q)}-
$$

As the functor from Ref. [52] is an equivalence for projectives/tilting modules, it follows that the fusion rules for the induction product of projective modules over $T L$ are the same as those for the direct summands (tilting modules) of the commutants at the corresponding values of $q$ and $m$. Since the induction product of projective TL modules closes on projective modules, and the tensor product of $\mathcal{A}$ or $U_{q}\left(\mathrm{sl}_{2}\right)$ tilting modules closes on tilting modules, these fusion rules can be characterized completely by specifying the multiplicities in the decomposition of a product of the respective indecomposable modules into indecomposables, just as in the semisimple case. So this result says that these multiplicities agree. This will be used extensively in the companion paper [13.

We can also ask if the product on the TL side is compatible with the lifting functor we defined above via the relation with the lift on modules of the commutant under $L \rightarrow L+1$. We need to show that one further square of functors commutes, namely the products agree, whether taken before or after the lift. Making use of the functor mapping to modules over the commutant, this is one face of a cube whose faces are commuting squares, for which we have already know that all the other squares commute (some by definition of the functors). It follows that this one does also, at least when restricted to projective TL modules.

\section{G. Closed chains}

We will briefly consider the closed chains in the not necessarily semisimple context also. The JTL algebra is cellular [28], and is semisimple when $q$ is not \pm 1 or a root of unity. $\mathrm{JTL}_{2 L}(q)$ is represented faithfully in our space $V$ provided $m+2 n>2$. For the commutant, we can apply similar methods as in the open case to show that the commutant in $V$ is also cellular. The standard modules have dimensions $\widehat{D}_{j K}^{\prime}$ for all $q$, (and superdimensions $\left.\widehat{D}_{j K}^{\prime}\right)$, and so the commutant has dimension $\operatorname{dim} \widehat{\mathcal{A}}_{m+n \mid n}=\sum_{j, K}\left(\widehat{D}_{j K}^{\prime}\right)^{2}$, where $j=0, \ldots, L$ as usual.

For the closed chains, the product operations, taking states of two closed chains to those of a single closed chain, can be thought of using a "pants" diagram (or trinion). In order to join two incoming legs into a single torso, it is necessary first to break each incoming closed chain, obtaining two open chains, then join these end to end, and finally close the other two ends. For the commutant algebra $\widehat{\mathcal{A}}_{m+n \mid n}\left(2 L_{1}\right)$ (and the others with $L_{2}$, $L=L_{1}+L_{2}$ ), this produces a series of operations: first induction from the closed to open case, for breaking each leg; then the tensor product in the open case; finally, the restriction map from open to closed algebras. For the modules over the JTL algebras, there is a product 
defined similarly (with induction and restriction interchanged). At least in the semisimple cases, the fusion rules for the JTL algebra and its commutant agree. Notice that, because of the use of induction, the product of $\widehat{\mathcal{A}}_{m+n \mid n}$ modules does not conserve dimensions (it is not a tensor product of vector spaces), and also that as $L \rightarrow \infty$ there is no upper limit on the $j$ values of the representations in the fusion rules, though there is necessarily such a limit on the highest weights in their Clebsch-Gordan decomposition into $U\left(\mathrm{gl}_{m}\right)$ modules. (Higher $j$ values are generated during the induction step from closed to open chains, as a given $j$ in the closed chain might arise from an arbitrarily higher (as $L_{1} \rightarrow \infty$ ) $j$ in an open chain, by making contractions to a singlet across the end of the chain.)

\section{CONTINUUM LIMIT AND CFTS}

In this last section before the conclusion, we comment a little on the continuum limits of the chains. This topic will be taken up in greater depth in a forthcoming paper [13].

In previous sections, we have considered the $L \rightarrow \infty$ of the algebraic structures in the chains, especially the commutant of the TL algebra and its modules, and the modules over the TL algebra, from a purely algebraic point of view. But from a physical point of view, more is required. Physically, we want to choose a Hamiltonian $H$ for the chain, and examine low-energy (and longwavelength) properties in the $L \rightarrow \infty$ limit. We will view the limit as taken with a lattice spacing distance tending to zero as $L \rightarrow \infty$, such that the length of the chain remains constant in the limit, equal to 1 , say (hence the term "continuum limit"), and also with all parameters in $H$ proportional to $L$. Then low energies and long wavelengths mean excitation energies and wavevectors of order 1 in these units. We are especially interested to begin with in cases where this continuum limit is a non-trivial conformal field theory, which in our units implies that excited states at energies of order 1 above the ground state do exist. For the supersymmetric chains $V$ considered here, or for the spin- $1 / 2$ chain, this occurs for the Hamiltonian $H=-L \sum_{i} e_{i}$, when $m$ is in the range $-2<m \leq 2$. It follows immediately from our analysis that our commutant algebra is a symmetry of the lowlying spectrum of this Hamiltonian for any finite $L$. It is not entirely clear how the limit can be taken in a mathematically rigorous way, but roughly we want to take the eigenvectors of $H$ that have low-energy eigenvalues, and we expect that the inner products among these vectors can be made to tend to some limits. Further, if we focus on long wavelength Fourier components of the set of $e_{i}$, then we expect their limits to exist, and their commutation relations to tend to those of the Virasoro generators $L_{n}\left(L_{n}+\bar{L}_{n}\right.$ in the closed chain case) [60], in the sense of weak convergence of matrix elements in this basis of low-energy eigenvectors. Then the modules over the TL algebra become modules over the UEA of the Virasoro, or possibly even a larger, algebra. (For the closed chains, two copies of the Virasoro algebra with generators $L_{n}$, $\bar{L}_{n}$ should eventually emerge.)

The symmetry (commutant) algebra in the continuum limit, which commutes with the Virasoro algebra, must be at least as large as that in the finite- $L$ chains. For the open chains, it appears that our commutant algebra [or $U_{q}\left(\mathrm{sl}_{2}\right)$ in the spin- $1 / 2$ chain] does not become even larger in the limit. (In certain cases, such as the open or closed $m=2$ spin- $1 / 2$ chain, the symmetry algebra combines with the Virasoro algebra to form the $\mathrm{sl}_{2}$ level 1 current [affine Lie] algebra, but this is not the case in general [8].) In the continuum limit, the basis for the commutant algebra takes a similar form as on the lattice, and can now be written for the open cases using

$$
\begin{aligned}
& \widetilde{J}_{b_{1} b_{2} \cdots b_{k}}^{a_{1} a_{2} \cdots a_{k}}= \\
& \quad \int_{0<x_{1}<x_{2}<\cdots<1} \prod_{i=1}^{k} d x_{i} J_{b_{1}}^{a_{1}}\left(x_{1}\right) J_{b_{2}}^{a_{2}}\left(x_{2}\right) \cdots J_{b_{k}}^{a_{k}}\left(x_{k}(56)\right.
\end{aligned}
$$

where the integration is over $0<x_{1}<x_{2}<\cdots<x_{k}<1$ (where $x L$ is position on the chain, and all operators are at the same time), $J_{b}^{a}(x)$ stands for the density of the generators $J_{i b}^{a}$ at $x$, and the contraction of any upper with a neighboring lower index is required to vanish as before (more precisely, one must use the same relations derived in the Appendix for the lattice case). The integration domain in these expressions resembles that for the generators of the Yangian in an integrable system, but we emphasize again that our algebra is not the Yangian. The definition can be generalized using an arbitrary Jordan curve $C$ with ends on the boundary (even with both ends on the same boundary), with $J_{b}^{a}(x)$ replaced by the component of the divergenceless currents $J_{\mu b}^{a}(\mu=1,2)$ normal to the curve, and the integrations are long the curve. These definitions also apply mutatis mutandis to the symmetry algebras of the closed chain, with an arbitrary closed Jordan curve $C$ in the most general form. These definitions for general curves ensure that the enlarged symmetry really commutes with conformal mappings of spacetime (which map curves $C$ onto one another), and generalize those for the global symmetry generators of $\mathrm{gl}(m+n \mid n)$, etc. [We note that while, unlike in theories with an affine Lie algebra, the Noether currents $J_{\mu b}^{a}$ do not possess a decomposition into purely holomorphic and anti-holomorphic parts [8], their flux across a curve is the Noether charge and is conserved and conformally invariant, and thus this is expected to hold for our operators also.]

Except for $m=2$, the theories are not semisimple, and we expect that the decomposition of the states under $\mathrm{TL} \otimes \mathcal{A}_{m+n \mid n}$ also determines the Virasoro structure. Hence the structures studied here in the finite chains should be very useful for the CFTs. In particular, much of the structure, including the fusion rules, is dictated by the symmetry [13]. 
We emphasize that in the limit, the commutant algebras commute with the full Virasoro algebra. We recall that a ("fully-extended") chiral algebra in a CFT is a maximal algebra of integer-conformal-spin holomorphic fields that have abelian monodromy and fusion rules. It seems that in most of the present cases, the chiral algebra is just the Virasoro algebra. It is unusual to find a large algebra that commutes with the full chiral algebra (the Yangians, when present, do not commute with the full chiral algebra, but only with a commutative subalgebra). There may be cases of rational CFTs in which a finite group commutes with the chiral algebra and fixes aspects of the CFT. But in the present cases, the symmetry algebra is infinite-dimensional. Thus we have begun the study of what we will, provisionally, call "CFTs with symmetry" in which some, possibly large, "global" symmetry algebra commutes with the chiral algebra of the CFT. We expect that a correct use of symmetry can be an important guiding principle in understanding irrational CFTs.

Some consequences of this procedure are worth emphasizing. For the open oriented-loops models, the cases $m$ and $-m(m \neq 0)$ were the same, because the TL algebras are isomorphic. The symmetry algebras were consequently also Morita equivalent, and this is a consequence of only $q^{2}$ (not $q$ ) entering expressions. But for the continuum limit defined here, a choice of Hamiltonian is an essential part of the construction of the limit. The Hamiltonian $H=-L \sum_{i} e_{i}$ is in isomorphic algebras in the two cases, but the isomorphism involves reversing the signs of all the generators $e_{i}$, and thus reversing the sign of $H$. Hence focusing on low energies in the two cases produces different continuum limits. For example, for $m=1$, the limiting CFT has central charge $c=0$, while for $m=-1$ it has $c=-7$. These two theories have the same commutant or symmetry structure, and the purely algebraic $L \rightarrow \infty$ limits of the modules, and the fusion rules, are the same, so that their Virasoro properties are still similar. [For $m=2$, the limit has $c=1$, and is related by Morita equivalence of symmetries to the limit of the antiferromagnetic spin- $1 / 2$ chain, which is a $c=1$ CFT, while for $m=-2$, the continuum limit is related to the $\mathrm{SU}(2)$ ferromagnet, and is not even conformal.] There are further Hamiltonians that may be of physical interest in the same chains, that are elements of the TL algebra, and act within constrained subspaces as in Ref. [61] and Sec. 6 of Ref. [9], and these can produce further distinct CFTs in the continuum limit. Similarly, the dilute-loops models also have continuum limits in a similar fashion, and have the same symmetry algebras commuting with the Virasoro algebra as in the corresponding dense-loops cases.

\section{CONCLUSION}

As this paper has covered a lot of ground, let us try to summarize a few points here. We began with simple models of spin chains with $m$ states per site $(m \geq 2)$, with nearest-neighbor interactions with $\mathrm{U}(m)$ symmetry (under which the sites transform alternately in the fundamental and its conjugate representation), the interaction being essentially the projection onto a $\mathrm{U}(m)$ singlet. We showed that such spin chains, even with arbitrary coefficients of these interactions, have a much larger symmetry algebra than $\mathrm{U}(m)$, with representations labeled by $j=0,1, \ldots, L$, for the $2 L$-site chain, and these are irreducible in the case of open chains (i.e., free boundary conditions). This means that the spectrum of the chain is a lot simpler than might have been expected, and can be computed more easily if this symmetry is exploited. There are similar results for supersymmetric chains with $\operatorname{gl}(m+n \mid n)$ symmetry of nearest-neighbor interactions $(m+n, n \geq 0$, and here again $|m| \geq 2)$, (open chain) Potts models in the Potts representation, and also for closed chains (i.e., periodic boundary conditions), though for the latter the representation structure is richer. The symmetries also apply to the loop models that can be obtained from the spin chains in a spacetime or transfer matrix picture, and there are similar ones for dilute loop models. In the loop language, the symmetries arise because the loops cannot cross.

Full use of symmetry in physics requires more structure than just an associative algebra. One wants to tensor representations and decompose the product into representations, and there should be dual representations for use as anti-particles. These structures were obtained here (for the open chains) by considering joining chains end to end. The "fusion" rules for decomposing the tensor product of representations labeled $j_{1}$ and $j_{2}$ took the same form as the Clebsch-Gordan series for SU(2). These structures turn the symmetry algebra into a Hopf algebra, and this is said to be Morita equivalent to a Hopf algebra $U_{q}\left(\mathrm{sl}_{2}\right)$ that is the quantum group deformation of the familiar $\mathrm{SU}(2)$ algebra. We also introduced structures of braiding and a twist; the former allows us to exchange representations, and obeys Yang-Baxter-type braid group relations. With the complete structure, one can compute, for example, $6 j$ symbols for the algebras.

Finally, we successfully extended all the open-chain results to the cases $|m|<2$, in the supersymmetric versions, for which the algebras are no longer semisimple (that is, representations are not fully decomposable into direct sums). This is of interest because such models arise in connection with, for example, disordered fermions, percolation, and polymers (self-avoiding walks), all in two dimensions. The cases $|m|<2$, for suitable Hamiltonians, possess continuum limits that are critical (conformal) field theories, or massive perturbations of the same. The structure of these open-chain conformal field theories is discussed in a companion paper [13].

The present paper culminated in the following result, which is worth stating again here: we find ribbon Hopf algebras $\mathcal{A}_{m+n \mid n}$ for all $m+n, n \geq 0$ (resp., $\mathcal{B}_{m+2 n \mid 2 n}$ for all $m+2 n, n \geq 0$ ) that are Morita equivalent as ribbon Hopf algebras to $U_{q}\left(\mathrm{sl}_{2}\right)$ restricted to integer spin finite- 
dimensional representations [resp., $U_{-q}\left(\mathrm{sl}_{2}\right)$ restricted to finite-dimensional representations, and with the variant twist map] for $m^{2}=\left(q+q^{-1}\right)^{2}$ (resp., $m=q+q^{-1}$ ). (The two cases arise in correspondence with loop models that respectively either have or do not have a fixed orientation on the loops.) These algebras are thus "quantum", even though the construction was very "classical". The algebras were also analyzed as cellular algebras [28].

A further result worth stating again concerns the Temperley-Lieb algebra, which is generated by the nearest-neighbor interaction terms in the spin chains. There is a notion of fusion for its representations also, induced by joining chains end to end. The fusion of projective modules closes on projective modules, and we proved that the fusion rules are the same as for the direct summand (or "tilting") modules for the corresponding symmetry algebras $\mathcal{A}$ or $\mathcal{B}$, whose fusion likewise closes on themselves, as for $U_{q}\left(\mathrm{sl}_{2}\right)$ [36]. For the continuum limit, when it is a conformal field theory, this gives the fusion rules for the corresponding conformal fields. This is considered further in the companion paper [13].

As open problems for future study, we have introduced a notion of "conformal field theories with symmetry", in which a large symmetry algebra such as our $\mathcal{A}_{m+n \mid n}$ commutes with the whole Virasoro algebra (or with some larger chiral algebra). This may be a key idea for understanding irrational conformal field theories such as those for the critical points of disordered fermions in two dimensions, as in the quantum Hall effect. More mathematical open problems would include searching for Hopf algebras - especially ones that are extensions of classical Hopf (super-) algebras, such as $U(\operatorname{gl}(m \mid n))$ or $U(\operatorname{osp}(m \mid 2 n))$ - that are Morita equivalent to other quantum groups. The corresponding notions starting from closed chains are also a subject in urgent need of study.

\section{Acknowledgments}

We are grateful to I. Frenkel and Z. Wang for helpful remarks, discussions, or correspondence. Work by NR was supported by NSF grant no. DMR-02-42949.

\section{APPENDIX A: SUPERALGEBRA CONSTRUCTIONS}

\section{Graded tensor products}

A $\mathbf{Z}_{2}$-grading on an associative algebra $\mathcal{A}$ can be introduced if $\mathcal{A}$ is a direct sum of two subspaces, $\mathcal{A}=$ $\mathcal{A}_{(0)} \oplus \mathcal{A}_{(1)}$, with $1 \in \mathcal{A}_{(0)}$, and we can associate with each element of these subspaces its degree, $\operatorname{deg} a=0$, 1 , for $a \in \mathcal{A}_{(0)}, \mathcal{A}_{(1)}$ respectively, such that $\operatorname{deg}\left(a_{1} a_{2}\right)=$ $\operatorname{deg} a_{1}+\operatorname{deg} a_{2}(\bmod 2)$, for all $a_{1}, a_{2}$ that lie in either $\mathcal{A}_{(0)}$ or $\mathcal{A}_{(1)}$, so that the degree map is a homomorphism of the multiplicative (monoid) structure of $\mathcal{A}$ into $\mathbf{Z}_{2}$ (viewed as a monoid under addition). The existence of such a grading implies that a grading can be defined on a module $\mathcal{V}$ over $\mathcal{A}$, by finding subspaces $\mathcal{V}_{(0)}$ and $\mathcal{V}_{(1)}$ (of degrees 0,1 respectively) with $\mathcal{V}=\mathcal{V}_{(0)} \oplus \mathcal{V}_{(1)}$, such that if $v$ is an element of either $\mathcal{V}_{(0)}$ or $\mathcal{V}_{(1)}$ and $a$ is an element of either $\mathcal{A}_{(0)}$ or $\mathcal{A}_{(1)}$, then $\operatorname{deg} a v=\operatorname{deg} a+\operatorname{deg} v(\bmod 2)$.

The grading plays a role in tensor products (we follow Kassel [26], but generalized to include grading). The tensor product of $\mathcal{A}$ with itself is defined as a vector space in the usual way, and similarly for the tensor product $\mathcal{V}_{1} \otimes \mathcal{V}_{2}$ of two modules (representations) $\mathcal{V}_{1}$ and $\mathcal{V}_{2}$ over $\mathcal{A}$. The latter becomes a representation of $\mathcal{A} \otimes \mathcal{A}$ by the action $\left(a_{1} \otimes a_{2}\right)\left(v_{1} \otimes v_{2}\right)=(-1)^{\operatorname{deg} a_{2} \operatorname{deg} v_{1}} a_{1} v_{1} \otimes a_{2} v_{2}$ when $a_{1}, a_{2}, v_{1}$ and $v_{2}$ lie in the subspaces on which the grading is defined. In particular, to make $\mathcal{A} \otimes \mathcal{A}$ a left (and right) module over itself, we must have $\left(a_{1} \otimes\right.$ $\left.a_{2}\right)\left(a_{3} \otimes a_{4}\right)=(-1)^{\operatorname{deg} a_{2} \operatorname{deg} a_{3}} a_{1} a_{3} \otimes a_{2} a_{4}$ when each of $a_{1}, \ldots, a_{4}$ lies in either $\mathcal{A}_{(0)}$ or $\mathcal{A}_{(1)}$. The grading on $\mathcal{V}_{1} \otimes \mathcal{V}_{2}$ itself is defined by $\operatorname{deg}\left(v_{1} \otimes v_{1}\right)=\operatorname{deg} v_{1}+\operatorname{deg} v_{2}$ for $v_{1} \in \mathcal{V}_{1(0)}$ or $\mathcal{V}_{1(1)}$, and similarly for $v_{2}$. (From here on we omit further specifications of elements or vectors as belonging to either one of the graded subspaces when it is obvious from the context that such a condition is needed in order that an expression containing the degree of an element or vector be well-defined.)

Finally, the flip map $\tau$ is a homomorphism of graded modules $\mathcal{V}_{1} \otimes \mathcal{V}_{2} \rightarrow \mathcal{V}_{2} \otimes \mathcal{V}_{1}$ such that $\tau^{2}=\mathrm{id}$, and in particular an involutory automorphism of $\mathcal{A} \otimes \mathcal{A}$. It is required to reduce to the usual flip map on the tensor product of the even (i.e. degree zero) subspaces. Hence it is given in general by $\tau\left(v_{1} \otimes v_{2}\right)=(-1)^{\operatorname{deg} v_{1} \operatorname{deg} v_{2}} v_{2} \otimes v_{1}$, and similarly on $\mathcal{A} \otimes \mathcal{A}$. The flip map is also used in defining the "opposite" algebra $\mathcal{A}^{\text {op }}$ (in which the order of multiplication is reversed), because multiplication can be regarded as a bilinear map from the vector space $\mathcal{A} \otimes \mathcal{A}$ to $\mathcal{A}$. So in $\mathcal{A}^{\text {op }}$, we first apply $\tau$ to $\mathcal{A} \otimes \mathcal{A}$, then multiply (in $\mathcal{A}$ ), to obtain $a \cdot b=(-1)^{\operatorname{deg} a \operatorname{deg} b} b a . \mathcal{A}$ is (graded) commutative if $a \cdot b=a b$ for all $a, b$ in $\mathcal{A}$.

More generally, if $f$ is a linear map of graded vector spaces, $f: \mathcal{V}_{1} \rightarrow \mathcal{V}_{2}$ that respects the grading, then we can define $\operatorname{deg} f=0$ if it maps $\mathcal{V}_{1(0)}$ to $\mathcal{V}_{2(0)}$ and $\mathcal{V}_{1(0)}$ to $\mathcal{V}_{2(0)}$, while $\operatorname{deg} f=1$ if it does the reverse. The space of all linear maps $\mathcal{V}_{1} \rightarrow \mathcal{V}_{2}$, called $\operatorname{Hom}\left(\mathcal{V}_{1}, \mathcal{V}_{2}\right)$, then becomes a graded vector space. If $f, g$ are linear maps of graded vector spaces, $f: \mathcal{V}_{1} \rightarrow \mathcal{V}_{2}$, and $g$ : $\mathcal{W}_{1} \rightarrow \mathcal{W}_{2}$, then on the tensor product we have $f \otimes g$ : $\mathcal{V}_{1} \otimes \mathcal{W}_{1} \rightarrow \mathcal{V}_{2} \otimes \mathcal{W}_{2}$ defined on $v_{1} \in \mathcal{V}_{1}$ and $v_{2} \in \mathcal{W}_{1}$ by $(f \otimes g)\left(v_{1} \otimes v_{2}\right)=(-1)^{\operatorname{deg} g \operatorname{deg} v_{1}} f v_{1} \otimes g v_{2}$.

\section{Dual spaces and spin chain}

In this subsection we address the left and right duals of a graded vector space, and the basic definition for our spin chain space $V$.

Because we write maps on the left, it is natural to define the (left) dual $\mathcal{V}^{*}$ of a graded vector space $\mathcal{V}$ to be the space of all graded $\mathbf{C}$-linear maps to the complex 
numbers, $\mathcal{V}^{*}=\operatorname{Hom}(\mathcal{V}, \mathbf{C})$. Equivalently, there is a dual pairing (or evaluation map) $d_{\mathcal{V}}: \mathcal{V}^{*} \otimes \mathcal{V} \rightarrow$ C. Note that this is similar to conventional Dirac notation in quantum mechanics, except that no complex conjugation is involved in the definition of the dual. There is also a "co-evaluation" map $b_{\mathcal{V}}: \mathbf{C} \rightarrow \mathcal{V} \otimes \mathcal{V}^{*}$ that is compatible with $d_{\mathcal{V}}$. It is defined via the natural identification of the action of $\mathbf{C}$ on $\mathcal{V}$ as elements in $\operatorname{End} \mathcal{V}=\operatorname{Hom}(\mathcal{V}, \mathcal{V})$, and End $\mathcal{V}$ is naturally isomorphic to $\mathcal{V} \otimes \mathcal{V}^{*}$ (all as graded objects, compatibly with the grading). Then $\mathcal{V}$ becomes a left module over End $\mathcal{V}$.

Given a map of graded spaces $f: \mathcal{V} \rightarrow \mathcal{W}$, and the dual spaces, we can define the super-transpose map $f^{*}$ : $\mathcal{W}^{*} \rightarrow \mathcal{V}^{*}$, such that $d_{\mathcal{V}}\left(f^{*} \otimes \mathrm{id}\right)=d_{\mathcal{W}}(\mathrm{id} \otimes f)$ on $\mathcal{W}^{*} \otimes \mathcal{V}$, which leads to $d_{\mathcal{V}}\left(f^{*}(\alpha) \otimes v\right)=(-1)^{\operatorname{deg} \alpha \operatorname{deg} f} d_{\mathcal{W}}(\alpha \otimes$ $f(v)$ ), where $v \in \mathcal{V}, \alpha \in \mathcal{W}^{*}$. This defines $f^{*}$ through its matrix elements, in analogy with the usual definition of the transpose of a matrix.

The right dual ${ }^{*} \mathcal{V}$ of a graded vector space can also be defined, via compatible maps $d_{\mathcal{V}}^{\prime}: \mathcal{V} \otimes{ }^{*} \mathcal{V} \rightarrow \mathbf{C}$ and $b_{\mathcal{V}}^{\prime}: \mathbf{C} \rightarrow{ }^{*} \mathcal{V} \otimes \mathcal{V}$. This may be identified with $\mathcal{V}^{*}$ through the flip map, $d_{\mathcal{V}}^{\prime}=d_{\mathcal{V}} \circ \tau$ and $b_{\mathcal{V}}^{\prime}=\tau \circ b_{\mathcal{V}}$. Now we can point out that if $\mathcal{V}^{*}$ is the left dual of $\mathcal{V}$, then $\mathcal{V}$ is the right dual of $\mathcal{V}^{*},{ }^{*}\left(\mathcal{V}^{*}\right) \cong\left({ }^{*} \mathcal{V}\right)^{*} \cong \mathcal{V}$ (canonical isomorphisms). Consequently, $\mathcal{V}^{* *}$ cannot be canonically identified with $\mathcal{V}$, as would be the case for ordinary vector spaces, though they are isomorphic. On elements, if we view a nonzero vector $v \in \mathcal{V}$ as part of a basis for $\mathcal{V}$, and define $v^{*}$ to be the dual basis vector, and similarly for $v^{* *}$, then the isomorphism maps $v^{* *} \in \mathcal{V}^{* *}$ to $(-1)^{\operatorname{deg} v} v \in \mathcal{V}$, because of the use of the flip map $\tau$ that relates the right and left duals.

Our supersymmetric "spin chain" can be constructed using these ideas. The space $V=V_{0} \otimes V_{1} \otimes \cdots V_{2 L-1}$, where $V_{i}$ is isomorphic to $V_{0}$ for $i$ even [ $V_{0}$ is referred to as the fundamental or defining representation of $\mathrm{gl}(m+$ $n \mid n)$ ], while $V_{i}$ for $i$ odd is viewed as the left dual of $V_{0}$. The definitions above are compatible with, and sufficient to establish, the constructions of the supersymmetric chains in Ref. 8]. The elements $e_{i}$ can be identified with $e_{i}=b_{V_{0}} d_{V_{0}}^{\prime}$ acting on the pair $i, i+1$ for $i$ even, and similarly for $i$ odd, where $V_{0}$ is the graded vector space of dimensions $m+n \mid n$ for the oriented loops models (for any non-negative values of $m+n$ and $n$ ).

\section{Explicit construction of spin chain and endomorphisms}

We now describe in detail the explicit construction of the space $V$ using boson and fermion oscillators with constraints. We also include the space of endomorphisms of the chain, and an anti-involution on these endomorphisms.

For $i$ even we have boson operators $b_{i}^{a}, b_{i a}^{\dagger},\left[b_{i}^{a}, b_{j b}^{\dagger}\right]=$ $\delta_{i j} \delta_{b}^{a}(a, b=1, \ldots, n+m)$, and fermion operators $f_{i}^{\alpha}$, $f_{i \alpha}^{\dagger},\left\{f_{i}^{\alpha}, f_{j \beta}^{\dagger}\right\}=\delta_{i j} \delta_{\beta}^{\alpha}(\alpha, \beta=1, \ldots, n)$; here labels like $\alpha$ on the fermion operators stand for $\alpha=a-(m+n)$ for a corresponding $a$ index. For $i$ odd, we have similarly boson operators $\bar{b}_{i a}, \bar{b}_{i}^{a \dagger},\left[\bar{b}_{i a}, \bar{b}_{j}^{b \dagger}\right]=\delta_{i j} \delta_{a}^{b}(a, b=1, \ldots$, $n+m)$, and fermion operators $\bar{f}_{i \alpha}, \bar{f}_{i}^{\alpha \dagger},\left\{\bar{f}_{i \alpha}, \bar{f}_{j}^{\beta \dagger}\right\}=$ $-\delta_{i j} \delta_{\alpha}^{\beta}(\alpha, \beta=1, \ldots, n)$. Notice the minus sign in the last anticommutator; since our convention is that the $\dagger$ stands for the adjoint, this minus sign implies that the norm-square of any two states that are mapped onto each other by the action of a single $\bar{f}_{i \alpha}$ or $\bar{f}_{i}^{\alpha \dagger}$ have opposite signs, and the "Hilbert" space has an indefinite inner product. The space $V$ is now defined as the subspace of states that obey the constraints

$$
\begin{aligned}
\sum_{a} b_{i a}^{\dagger} b_{i}^{a}+\sum_{\alpha} f_{i \alpha}^{\dagger} f_{i}^{\alpha}=1 \quad(i \text { even }), \\
\sum_{a} \bar{b}_{i}^{a \dagger} \bar{b}_{i a}-\sum_{\alpha} \bar{f}_{i}^{\alpha \dagger} \bar{f}_{i \alpha}=1 \quad(i \text { odd }) .
\end{aligned}
$$

The sums here and below are over $a=1, \ldots, m+n$, and $\alpha=1, \ldots, n$; for clarity, we are not using the summation convention on indices $a, \alpha$ in this Appendix.

The generators of the Lie superalgebra $\operatorname{gl}(m+n \mid n)$ acting on each site of the chain are the bilinear forms $J_{i a}^{b}=b_{i a}^{\dagger} b_{i}^{b}, f_{i \alpha}^{\dagger} f_{i}^{\beta}, b_{i a}^{\dagger} f_{i}^{\beta}, f_{i \alpha}^{\dagger} b_{i}^{b}$ (depending on whether $a$, $b$ on the left hand side are in $1, \ldots, m+n$ or $m+n+1, \ldots$, $m+2 n)$ for $i$ even, and correspondingly $J_{i a}^{b}=-\bar{b}_{i}^{b \dagger} \bar{b}_{i a}$, $\bar{f}_{i}^{\beta \dagger} \bar{f}_{i \alpha},-\bar{f}_{i}^{\beta \dagger} \bar{b}_{i a},-\bar{b}_{i}^{b \dagger} \bar{f}_{i \alpha}$ for $i$ odd, which for each $i$ have the same (anti-)commutators as those for $i$ even. Under the transformations generated by these operators, $b_{i a}^{\dagger}, f_{i \alpha}^{\dagger}$ ( $i$ even) transform as the fundamental (defining) representation $V_{0}$ of $\operatorname{gl}(n+m \mid n), \bar{b}_{i}^{a \dagger}, \bar{f}_{i}^{\alpha \dagger}$ ( $i$ odd) as the (left) dual fundamental $V_{0}^{*}$. Hence the space $V$ is the graded tensor product of alternating irreducible representations $V_{0}, V_{0}^{*}$ as desired; the signs in the $J_{i a}^{b}$ for $i$ odd can be understood as these generators are minus the supertranspose of the action on the fundamental. In the spaces $V_{0}^{*}$ on the odd sites, the odd states (those with fermion number $-\bar{f}_{i}^{\alpha \dagger} \bar{f}_{i \alpha}$ equal to one) have negative norm-square. The destruction operators $b_{i}^{a}, f_{i}^{\alpha}$ on the even sites transform in the dual $V_{0}^{*}$. Those on the odd sites $\bar{b}_{i a}, \bar{f}_{i \alpha}$ do not transform in the double dual $V_{0}^{* *}$, which is the dual of $V_{0}^{*}$ and would be obtained if the anticommutator for $\bar{f}$ contained the usual plus sign. Instead, because of the minus in the relevant anticommutators, they transform in $V_{0}$. Thus upper (resp., lower) indices always transform in the same way. (We note that for the $\operatorname{osp}(m+2 n \mid 2 n$ ) spin chains or unoriented loops models, in which all sites are supposed to be equivalent, the negative signs can be assigned to $n$ of the $2 n$ fermion components on every site, in a translationally-invariant fashion, with similar results.)

The TL generators are constructed as follows. First, we note that for any two sites $i$ (even), $j$ (odd), the combinations

$$
\sum_{a} \bar{b}_{j a} b_{i}^{a}+\sum_{\alpha} \bar{f}_{j \alpha} f_{i}^{\alpha}, \quad \sum_{a} b_{i a}^{\dagger} \bar{b}_{j}^{a \dagger}+\sum_{\alpha} f_{i \alpha}^{\dagger} \bar{f}_{j}^{\alpha \dagger}
$$


are invariant under $\operatorname{gl}(n+m \mid n)$. (The order of the operators in these expressions differs from Refs. [8, 9], but so do some signs in the expressions for the generators of $\operatorname{gl}(n+m \mid n)$ above, so that invariance still holds.) Then the evaluation and co-evaluation maps applied to each pair of neighbors $i, i+1$ can be written in terms of such combinations:

$$
\begin{aligned}
d_{V_{i+1}} & =\sum_{a} \bar{b}_{i+1, a} b_{i}^{a}+\sum_{\alpha} \bar{f}_{i+1, \alpha} f_{i}^{\alpha} \quad(i \text { even }),(\mathrm{A} 4) \\
d_{V_{i+1}} & =\sum_{a} \bar{b}_{i a} b_{i+1}^{a}+\sum_{\alpha} \bar{f}_{i \alpha} f_{i+1}^{\alpha} \quad(i \text { odd }), \quad(\mathrm{A} 5) \\
b_{V_{i}} & =\sum_{a} b_{i a}^{\dagger} \bar{b}_{i+1}^{a \dagger}+\sum_{\alpha} f_{i \alpha}^{\dagger} \bar{f}_{i+1}^{\alpha \dagger} \quad(i \text { even }), \quad(\mathrm{A} 6) \\
b_{V_{i}} & =\sum_{a} b_{i+1, a}^{\dagger} \bar{b}_{i}^{a \dagger}+\sum_{\alpha} f_{i+1, \alpha}^{\dagger} \bar{f}_{i}^{\alpha \dagger} \quad(i \text { odd }) .(\mathrm{A} 7)
\end{aligned}
$$

As $V_{0}^{* *}$ does not appear in $V$, we have used the isomorphism of $V_{0}^{* *}$ with $V_{0}$ (or equivalently of $V_{0}^{*}$ with ${ }^{*} V_{0}$ ) in the cases of $d_{V_{i+1}}$ for $i$ even and $b_{V_{i}}$ for $i$ odd; we have abused notation a little and not recorded this in the notation. (More accurately, we could write the operators for the latter two cases as $d_{V_{0}}^{\prime}$ and $b_{V_{0}}^{\prime}$, with the understanding that sites $i, i+1$ are meant with $V_{0}, V_{0}^{*}$ in their correct positions in the chain; this produces the correct signs.) Then the TL generators can be written as

$$
e_{i}=b_{V_{i}} d_{V_{i+1}}
$$

for all $i$.

Next we describe the endomorphisms of $V$ in terms of explicit expressions. We will revert to using indices $a=1$, $\ldots, m+2 n$, and it will be less confusing to drop the use of raised indices for the time being. Then we define a basis for the space of endomorphisms by the operators

$$
\begin{aligned}
& E_{a_{0}, a_{1}, \ldots, a_{2 L-1}, a_{0}^{\prime}, \ldots, a_{2 L-1}^{\prime}}= \\
& b_{0 a_{0}}^{\dagger} \bar{b}_{1}^{a_{1} \dagger} \cdots \bar{b}_{2 L-1}^{a_{2 L-1} \dagger} \bar{b}_{2 L-1, a_{2 L-1}^{\prime}} \cdots b_{0}^{a_{0}^{\prime}}
\end{aligned}
$$

These are written for the case all $a_{i}, a_{i}^{\prime}$ in the range 1 , $\ldots, m+n$; for any $a_{i}$ ( $i$ even) that lie in $m+n+1$, $\ldots, m+2 n, b_{i a}^{\dagger}$ must be replaced by $f_{i \alpha}^{\dagger}$, and similarly for $i$ odd and for $a_{i}^{\prime}$. We will simplify notation and write $\operatorname{deg} a=0$ for the degree of boson or fermion operators with $a$ in the range $1, m+n, \operatorname{deg} a=0$ for $a$ in $m+n+1$, $\ldots, m+2 n$. Then the multiplication of the $E_{\ldots} \mathrm{s}$ is given by

$$
\begin{aligned}
& E_{a_{0} \ldots a_{2 L-1}, a_{0}^{\prime} \ldots a_{2 L-1}^{\prime}} E_{b_{0} \ldots b_{2 L-1}, b_{0}^{\prime} \ldots b_{2 L-1}^{\prime}}= \\
& E_{a_{0} \ldots a_{2 L-1}, b_{0}^{\prime} \ldots b_{2 L-1}^{\prime}}(-1)^{\sum_{i \text { odd }} \operatorname{deg} a_{i}^{\prime}} \prod_{i} \delta_{a_{i},\left(, \hat{a}_{i} 10\right)}
\end{aligned}
$$

The minus signs can be removed by defining $\widetilde{E}_{a_{0} \ldots a_{2 L-1}^{\prime}}=$ $E_{a_{0} \ldots a_{2 L-1}^{\prime}}(-1)^{\sum_{i \text { odd }} \operatorname{deg} a_{i}^{\prime}}$. Then the $\widetilde{E}_{\ldots}$ s are a basis for the space of endomorphisms End $V$, which is naturally isomorphic to $V \otimes V^{*}$, and as the dual $V^{*}$ of $V=V_{0} \otimes$
$V_{0}^{*} \otimes \cdots$ is naturally isomorphic to $V_{0}^{* *} \otimes V_{0}^{*} \otimes \cdots$ with the reverse ordering, the $\widetilde{E}_{\ldots}$ s refer to the natural basis for this space. Again, the minus signs for odd elements on odd sites are due to the map from $V_{0}^{* *}$ to $V_{0}$, which are used to map $V^{*}$ to $V$.

The anti-isomorphism on the endomorphisms is given by

$$
\begin{aligned}
& \left(E_{a_{0} \ldots a_{2 L-1}^{\prime}}\right)^{s}= \\
& \quad(-1)^{\left(\sum_{i} \operatorname{deg} a_{i}\right)\left(\sum_{i^{\prime}} \operatorname{deg} a_{i}^{\prime}\right)} E_{a_{2 L-1}^{\prime} \ldots a_{0}} .
\end{aligned}
$$

One can check that this is an algebra isomorphism to $(\text { End } V)^{\text {op }}$ as required. Further, it is clear that $\left(\left(E_{a_{0} \ldots a_{2 L-1}^{\prime}}\right)^{s}\right)^{s}=E_{a_{0} \ldots a_{2 L-1}^{\prime}}$, so ${ }^{s}$ is an anti-involution. The sign in the definition of $s$ resembles that in the supertranspose $\left(E_{a_{0} \ldots a_{2 L-1}^{\prime}}\right)^{*}$ of $E_{a_{0} \ldots a_{2 L-1}^{\prime}}$, however the supertranspose of an endomorphism of $V$ would be an endomorphism of $V^{*}$ (acting on the left). Our antiisomorphism ${ }^{s}$ is obtained by combining the supertranspose with the map $V^{*} \rightarrow V$ given by multiplication by $(-1)^{\sum_{i \text { odd }} \operatorname{deg} a_{i}}$.

One can easily check that the identity in $\operatorname{End} V$,

$$
1=\sum_{a_{0} \ldots a_{2 L-1}}(-1)^{\sum_{i \text { odd }} \operatorname{deg} a_{i}} E_{a_{0} \ldots a_{2 L-1}, a_{0} \ldots a_{2 L-1}}
$$

is invariant under ${ }^{s}$, and that the TL generators map as $e_{i} \rightarrow e_{i}^{s}=e_{2 L-2-i}$. The anti-isomorphism $s$ is not determined uniquely by these properties. One can obtain other anti-isomorphisms by conjugating with, for example, elements of the supergroup $\mathrm{GL}(m+n \mid n)$, obtained by exponentiating the action of the generators $\sum_{i} J_{i a}^{b}$ of $\mathrm{gl}(m+n \mid n)$ on $V$. A particular case is the involutive automorphism of End $V$ given by multiplication of an element by -1 to its degree, which is conjugation by the similarly-defined map on $V$. However, this freedom may be reduced if we also insist that the anti-isomorphism be an anti-involution.

\section{Symmetry generators}

Now we obtain an explicit description of a set of operators that commute with the action of the TL algebra in $V$, which is shown in the main text to be a basis for the commutant algebra $\mathcal{A}_{m+n \mid n}(2 L)$. We again begin with a set of operators (for $k \leq 2 L$ )

$$
\widetilde{J}_{b_{1} b_{2} \ldots b_{k}}^{a_{1} a_{2} \ldots a_{k}}=\sum_{0 \leq i_{1}<i_{2}<\cdots<i_{k} \leq 2 L-1} J_{i_{1} b_{1}}^{a_{1}} J_{i_{2} b_{2}}^{a_{2}} \cdots J_{i_{k} b_{k}}^{a_{k}},
$$

where $J_{i a}^{b}$ for $a, b=1, \ldots, m+2 n$ have been defined above. As the notation may suggest, each $J_{i a}^{b}$ transforms as the representation $V_{0} \otimes V_{0}^{*}$ (with the graded tensor product in that order), whether $i$ is odd or even. This is clear for $i$ even as $J_{i a}^{b}=b_{i a}^{\dagger} b_{i}^{b}$ [again here, the notation is that $b_{i a}$ (resp., $\bar{b}_{i}^{a}$ ) means $f_{i \alpha}$ (resp., $\bar{f}_{i}^{\alpha}$ ) with 
$\alpha=a-(m+n)$ when $\alpha>0$, and similarly for the adjoints of these operators]. For $i$ odd there is an additional sign: $J_{i a}^{b}=-(-1)^{(\operatorname{deg} a)(\operatorname{deg} b)} \bar{b}_{i}^{b \dagger} \bar{b}_{i a}$. The overall minus is irrelevant, while $(-1)^{(\operatorname{deg} a)(\operatorname{deg} b)}$ is exactly the sign produced by flipping the order of the factors in the tensor product. Hence the set of $\widetilde{J}_{\mathrm{S}}$ for each $k$ transform as $V_{0} \otimes V_{0}^{*} \otimes \cdots \otimes V_{0}^{*}$, with $2 k$ factors, and in particular this is true for all terms in the summation over the $i_{l}$ s.

The $\widetilde{J}_{\mathrm{s}}$ are to be used as a basis set for a space of operators within which we construct the commutant of the TL algebra. It will suffice to consider linear combinations from a subset all having the same value of $k$. Then a linear combination may be written

$$
\widehat{j}=\sum_{a_{1}, a_{2}, \ldots, b_{k}} j_{a_{1} \ldots a_{k}}^{b_{1} \ldots b_{k}} \widetilde{J}_{b_{1} \ldots b_{k}}^{a_{1} \ldots a_{k}},
$$

where $j_{a_{1} \ldots a_{k}}^{b_{1} \ldots b_{k}}$ are numerical coefficients. For each $i$ in the range $i=0, \ldots, 2 L-2$, the terms in the summation over $i_{l} \mathrm{~s}$ in $\widehat{j}$ can be grouped into those in which zero, one, or two of the sites $i, i+1$ are occupied by a factor $J_{i}$ or $J_{i+1}$. Those in which none of the $i_{l},(l=1, \ldots, k)$ are equal to $i$ or $i+1$ clearly commute with $e_{i}$. Also, the terms in which just one $i_{l}=i$ or $i+1$ may be grouped in pairs, such that they contain $J_{i b_{l}}^{a_{l}}+J_{i+1, b_{l}}^{a_{l}}$ as a factor (all other operator factors commuting with $e_{i}$ ), and these also commute with $e_{i}$; indeed, they annihilate it from either side. Finally there are terms in $\widehat{j}$ in which $i_{l}=i$, $i_{l+1}=i+1$ for some $l$. For these it will clearly be sufficient if the linear combination of expressions containing $J_{i b_{l}}^{a_{l}} J_{i+1, b_{l+1}}^{a_{l+1}}$ annihilates $e_{i}$ from either side. (In the main text, the use of this stronger condition is motivated, and shown to produce the whole commutant.) This condition reduces further to the conditions that $d_{V_{i+1}}$ annihilates these terms from the left, and $b_{V_{i}}$ annihilates them from the right. This imposes a set of linear relations on the coefficients $j$. (It is here that the above transformation properties of the terms in $\widetilde{J}$ are important, as the coefficients $j$ contain no $i_{l}$ dependence.) These relations involve adjacent indices of the coefficients $j$. By explicit calculation we find:

$$
\begin{aligned}
\sum_{a} j_{\ldots a a_{l+1} \ldots} \ldots b_{l} a \ldots & =0(\mathrm{~A} 15) \\
\sum_{a} j_{\ldots a a_{l} a \ldots} a b_{l+1} \ldots(-1)^{\operatorname{deg} a+\operatorname{deg} a\left(\operatorname{deg} a_{l}+\operatorname{deg} b_{l+1}\right)} & =0(\mathrm{~A} 16)
\end{aligned}
$$

for $l=1, \ldots, k-1$; indices not displayed (those with subscript $<l$ or $>l+1)$ are free. The number of independent solutions to these relations for $k$ even is $\left(D_{k / 2}^{\prime}\right)^{2}$. This can be shown by using the independence of the coefficients from the length $L$, so that we may consider a chain with $k=2 L$; then reorder the operators in the $\widetilde{J}_{\mathrm{S}}$ into the form of the basis elements $E_{\ldots}$ above (this is an isomorphism), and consider the subspace annihilated by all $e_{i}$ from either side; as discussed in the main text, the dimension of this space is clearly the square of the dimension $D_{k / 2}^{\prime}$ of the standard module $\mathcal{V}_{k / 2}$.
In each space $J^{(k)}$ of operators constructed using solutions to the relations eqs. A15 and (A16), we can attempt to produce a complete set of operators $J_{b_{1} \ldots b_{k}}^{a_{1} \ldots a_{k}}$ that are not linearly independent, but obey relations on adjacent indices similar to those for $j$ (this is how these spaces of operators are referred to in the main text). These are supposed to be constructed (and normalized) in the form $J_{b_{1} \ldots b_{k}}^{a_{1} \ldots a_{k}}=\widetilde{J}_{b_{1} \ldots b_{k}}^{a_{1} \ldots a_{k}} \pm$ corrections that involve contractions of pairs of adjacent indices. From the $\operatorname{gl}(m+n \mid n)$ symmetry considerations above, the relations must take the form

$$
\begin{aligned}
\sum_{a} J_{\ldots b_{l} a \ldots}^{\ldots a a_{l+1} \ldots}(-1)^{\operatorname{deg} a} & =0 \\
\sum_{a} J_{\ldots a_{l} a \ldots}(-1)^{\operatorname{deg} a\left(\operatorname{deg} a_{l}+\operatorname{deg} b_{l+1}\right)} & =0
\end{aligned}
$$

for $l=1, \ldots, k-1$. For $n=0$ (and thus $m \geq 2$ ), these reduce to the tracelessness conditions used in the earlier sections of this paper. This works also for $n>0$, at least when $|m| \geq 2$, when the sets of $j$-coefficients involved are essentially Jones-Wenzl projectors acting in the index spaces; these spaces are isomorphic to $V(k) \otimes V(k)^{*}$. For example, for $k=2$ the expression is, for $m \neq 0$,

$$
\begin{aligned}
& J_{b_{1} b_{2}}^{a_{1} a_{2}=} \widetilde{J}_{b_{1} b_{2}}^{a_{1} a_{2}}-\frac{1}{m} \delta_{b_{2}}^{a_{1}} \sum_{a} \widetilde{J}_{b_{1} a}^{a a_{2}}(-1)^{\operatorname{deg} a} \\
&- \frac{1}{m} \delta_{b_{1}}^{a_{2}}(-1)^{\operatorname{deg} a_{2} \operatorname{deg} b_{1}+\operatorname{deg} a_{1} \operatorname{deg} b_{1}+\operatorname{deg} a_{2} \operatorname{deg} b_{2}} \\
& \times \sum_{a} \widetilde{J}_{a b_{2}}^{a_{1} a}(-1)^{\operatorname{deg} a\left(\operatorname{deg} a_{1}+\operatorname{deg} b_{2}\right)} \\
&+ \frac{1}{m^{2}} \delta_{b_{2}}^{a_{1}} \delta_{b_{1}}^{a_{2}}(-1)^{\operatorname{deg} a_{2} \operatorname{deg} b_{1}+\operatorname{deg} a_{1} \operatorname{deg} b_{1}+\operatorname{deg} a_{2} \operatorname{deg} b_{2}} \\
& \times \sum_{a, b} \widetilde{J}_{b a}^{a b}(-1)^{\operatorname{deg} a}
\end{aligned}
$$

this satisfies relations (A17), A18, and the coefficients $j$ in its expansion in terms of $\widetilde{J}_{\mathrm{S}}$ obey relations (A15), (A16). Obviously this expression fails for $m=0$. There are similar problems for some other $k$ values for the cases $m=0, \pm 1$, in which the algebras, and some of the standard modules, are not semisimple; the problem occurs whenever the $k / 2$ th standard TL module over $\mathrm{TL}_{k}(q)$ is not simple, in which case there is no corresponding JonesWenzl projector (an idempotent element of the $\mathrm{TL}_{k}(q)$ algebra) onto that module. When a complete set $J_{b_{1} \ldots b_{k}}^{a_{1} \ldots a_{k}}$ obeying all the conditions stated above does not exist for some value of $k$, the construction using coefficients $j$ can always be used instead.

For the action of the anti-involution on the symmetry generators, we first note that by writing $J_{i a}^{b}$ in terms of the basis elements $E_{\ldots}$, we can show that $\left(J_{i b}^{a}\right)^{s}=$ $-J_{2 L-1-i, b}^{a}$. Then from the anti-involution property of ${ }^{s}$,

$$
\begin{aligned}
\left(\widetilde{J}_{b_{1} \ldots b_{k}}^{a_{1} \ldots a_{k}}\right)^{s}= & (-1)^{k}(-1)^{\sum_{l<l^{\prime}}\left(\operatorname{deg} a_{l}+\operatorname{deg} b_{l}\right)\left(\operatorname{deg} a_{l^{\prime}}+\operatorname{deg} b_{l^{\prime}}\right)} \\
& \times \widetilde{J}_{b_{k} \ldots b_{1}}^{a_{k} \ldots a_{1}} .
\end{aligned}
$$


We can then see that the relations (A15), (A16) on the coefficients $j$ in the operators $\widehat{j}$ are invariant under ${ }^{s}$, and so the transformation maps $J^{(k)}$ into itself. One may obtain an idea of how the transformation looks from the action on the $J_{b_{1} \ldots b_{k}}^{a_{1} \ldots a_{k}}$, when these are available, which takes the same form as for $\widetilde{J}_{b_{1} \ldots b_{k}}^{a_{1} \ldots a_{k}}$.

\section{Hopf superalgebras}

When the definitions for superalgebras and graded tensor products of their modules are used to replace the usual ones [25, 26] in the definitions for Hopf algebras, ribbon Hopf algebras, etc, we obtain what may be called Hopf superalgebras, ribbon Hopf superalgebras, etc. We note that Hopf superalgebras arise naturally as the UEAs for Lie superalgebras, and the constructions for the action of $\operatorname{gl}(m+n \mid n)$ within the modules $V$ serve as examples. Needless to say, a Hopf superalgebra $\mathcal{A}$ for which $\mathcal{A}_{(1)}=0$ is just an ordinary Hopf algebra. Here we will mention the main variations in the definitions and properties for Hopf superalgebras.

First, the comultiplication $\Delta: \mathcal{A} \rightarrow \mathcal{A} \otimes \mathcal{A}$ is a superalgebra homomorphism; we write

$$
\Delta(a)=\sum_{(a)} a^{\prime} \otimes a^{\prime \prime} .
$$

There are unit $\eta: \mathbf{C} \rightarrow \mathcal{A}$ and counit $\varepsilon: \mathcal{A} \rightarrow \mathbf{C}$ maps, as usual; these maps are even. The antipode $S$ obeys

$$
\sum_{(a)} a^{\prime} S\left(a^{\prime \prime}\right)=\sum_{(a)} S\left(a^{\prime}\right) a^{\prime \prime}=\eta(\varepsilon(a))
$$

and is an anti-linear homomorphism to $\mathcal{A}^{\mathrm{op}}$, thus $S(a b)=$ $(-1)^{\operatorname{deg} a \operatorname{deg} b} S(b) S(a)$. If it is invertible, and if $S^{-1}=S$, as in our cases, then it is an anti-involution. Dually, $S$ is also a graded co-algebra homomorphism to the opposite co-algebra, in which the coproduct is $\Delta^{\mathrm{op}}=\tau \circ \Delta$; this means that it obeys $S \otimes S \circ \Delta^{\mathrm{op}}(a)=\Delta \circ S(a)$, which is

$$
\sum_{(a)}(-1)^{\operatorname{deg} a^{\prime} \operatorname{deg} a^{\prime \prime}} S\left(a^{\prime \prime}\right) \otimes S\left(a^{\prime}\right)=\sum_{(a)} S(a)^{\prime} \otimes S(a)^{\prime \prime} .
$$

The antipode is used in defining dual representations. For example, if $\mathcal{A}$ acts on $\mathcal{V}$ as $v \rightarrow$ av for $a \in \mathcal{A}$, $v \in \mathcal{V}$, then the left dual vector space $\mathcal{V}^{*}$ of $\mathcal{V}$ becomes the left dual as an $\mathcal{A}$-module, on which the $\mathcal{A}$ action is $w \rightarrow S(a)^{*} w$, where $w \in V^{*}$ and we used the supertranspose $S(a)^{*}$ of $S(a)$ so that $\mathcal{V}^{*}$ becomes a left $\mathcal{A}$-module. Readers can check that these definitions are satisfied by the UEA of $\operatorname{gl}(m+n \mid n)$, with the comultiplication and antipode defined on the generators $J_{a}^{b}$ by $\Delta\left(J_{a}^{b}\right)=J_{a}^{b} \otimes 1+1 \otimes J_{a}^{b}$ and $S\left(J_{a}^{b}\right)=-J_{a}^{b}$, and then extended to the rest of the algebra by the homomorphism properties, and also that this agrees with the construction of our spin chain. In particular, the odd sites indeed transform as the left dual of $V_{0}$.
The definitions of braiding and twist are unchanged when written in terms of the graded $\Delta^{\mathrm{op}}$, and so on, and with these additional structures we are led to definitions of ribbon Hopf superalgebras. The definitions of tensor and ribbon categories (as abstract categories) are unchanged, and are satisfied by the categories of graded modules over ribbon Hopf superalgebras, and hence equivalences of these categories still make sense in this broader context. The ordinary supertrace and superdimension on modules over the UEA of a Lie superalgebra can then be viewed as examples of the more general concepts of quantum trace and dimension.

\section{Enlarged symmetry algebra for open $\operatorname{gl}(1 \mid 1)$ chain}

Here we give details of our construction for the open $\operatorname{gl}(1 \mid 1)$ spin chain, which is a free fermion system [8]. The free fermion form of the model is defined using fermion operators $f_{i}$ and their adjoints $f_{i}^{\dagger}, i=0,1, \ldots, 2 L-1$, which obey $\left\{f_{i}, f_{i^{\prime}}\right\}=0,\left\{f_{i}, f_{i^{\prime}}^{\dagger}\right\}=(-1)^{i} \delta_{i i^{\prime}}$. The TL generators can be written as

$$
e_{i}=\left(f_{i}^{\dagger}+f_{i+1}^{\dagger}\right)\left(f_{i}+f_{i+1}\right),
$$

for $i=0, \ldots, 2 L-2$. Some symmetry operators in $J^{(k)}$, for $k=1,2$, which commute with all the $e_{i}$ s, are

$$
\begin{aligned}
F & =\sum_{i} f_{i}, \\
F^{\dagger} & =\sum_{i} f_{i}^{\dagger}, \\
F_{(2)} & =\sum_{i<i^{\prime}} f_{i} f_{i^{\prime}}, \\
F_{(2)}^{\dagger} & =\sum_{i<i^{\prime}} f_{i^{\prime}}^{\dagger} f_{i}^{\dagger}, \\
N & =\sum_{i}(-1)^{i} f_{i}^{\dagger} f_{i}-L .
\end{aligned}
$$

The remaining symmetry operators turn out to be sums of products of these, so this set of five operators (together with 1 ) is a set of generators of the full algebra $\mathcal{A}_{1 \mid 1}$. The graded commutators of these five close on themselves, so they form a Lie superalgebra. $F, F^{\dagger}$ generate a Lie subsuperalgebra isomorphic to $\operatorname{psl}(1 \mid 1) . F_{(2)}, F_{(2)}^{\dagger}$, and $N$ generate an $\mathrm{sl}_{2}$ Lie subalgebra, with $N$ as $2 S_{z}$, and $F$, $F^{\dagger}$ transform as a doublet under this $\mathrm{sl}_{2}$, and so form a Lie ideal. (We note that $(-1)^{i} f_{i}^{\dagger} f_{i}$ is the fermion number $=0,1$ at site $i$.) The Lie superalgebra is thus not semisimple, but is a semidirect product of these two, and can be viewed as the superalgebra of translations and $\mathrm{sl}_{2}$ rotations of the superplane with anticommuting coordinates $f, f^{\dagger}$. The associative (universal enveloping) algebra it generates, $\mathcal{A}_{1 \mid 1}$, is isomorphic to $U_{q}\left(\mathrm{sl}_{2}\right)$ (modulo the restriction to modules with integer $j$ ). Representations of this algebra acting in the spin chain can be easily constructed. 
For the corresponding closed chain, generators like $F_{(2)}$ are lost, as the summation must be extended around the chain, and then anticommutation of $f_{i}$ and $f_{i^{\prime}}$ makes it vanish.

We emphasize that for open $\operatorname{gl}(n \mid n)$ chains with $n>1$, the symmetry algebra $\mathcal{A}_{n \mid n}$ is not an enveloping algebra of a finite-dimensional Lie superalgebra. For the closed chains, some symmetry operators of the open version are lost on closing the chain, for reasons similar to the case of $F_{(2)}$ above, but for general $n>1$ many operators remain.
[1] R. Baxter, Exactly Solved Models in Statistical Mechanics (Academic Press, New York, 1982).

[2] I.A. Gruzberg, A.W.W. Ludwig, and N. Read, Phys. Rev. Lett. 82, 4524 (1999).

[3] B. Nienhuis, Phys. Rev. Lett. 49, 1062 (1982).

[4] P. di Francesco, H. Saleur, and J.B. Zuber, J. Stat. Phys. 49, 57 (1987).

[5] B. Nienhuis, J. Stat. Phys. 34, 731 (1984).

[6] M.N. Barber and M.T. Batchelor, Phys. Rev. B 40, 4621 (1989).

[7] I. Affleck, J. Phys. Cond. Matt. 2, 405 (1990).

[8] N. Read and H. Saleur, Nucl. Phys. B 613, 409 (2001) arXiv:hep-th/0106124.

[9] P. Fendley and N. Read, J. Phys. A 35, 10675 (2002) arXiv:hep-th/0207176.

[10] P. Di Francesco, P. Mathieu, and D. Senechal, Conformal Field Theory (Springer, New York, 1997).

[11] V. Gurarie, Nucl. Phys. B 410, 535 (1993).

[12] N. Mann and J. Polchinski, hep-th/0408162 Phys. Rev. D72, 086002 (2005) arXiv:hep-th/0508232.

[13] N. Read and H. Saleur, hep-th/0701xxx.

[14] F.D.M. Haldane and D.J. Arovas, private communication (1991).

[15] J.L. Jacobsen, N. Read, and H. Saleur, Phys. Rev. Lett. 90, 090601 (2003).

[16] H.N.V. Temperley and E.H. Lieb, Proc. Roy. Soc. (London) A 322, 251 (1971).

[17] For our purposes, a module (modules are left modules unless stated otherwise) is the same as a representation in a vector space over $\mathbf{C}$.

[18] R.S. Pierce, Associative Algebras, Graduate Texts in Mathematics 88 (Springer-Verlag, New York, NY, 1982).

[19] F.W. Anderson and K.R. Fuller, Rings and Categories of Modules, Graduate Texts in Mathematics 13 (2nd Ed., Springer-Verlag, New York, NY, 1992).

[20] V.F.R. Jones, Invent. Math. 72, 1 (1983).

[21] F.M. Goodman, P. de la Harpe, and V.F.R. Jones, Coxeter Graphs and Towers of Algebras, MSRI 14 (SpringerVerlag, New York, NY, 1989), Ch. 2.

[22] P.P. Martin, Potts Models and Related Problems in Statistical Mechanics (World Scientific, Singapore, 1991).

[23] B.E. Sagan, The Symmetric Group: Representations, Combinatorial Algorithms, and Symmetric Functions, 2nd Ed., Graduate Texts in Mathematics 203 (Springer, New York, NY, 2001).

[24] These are reviewed in M. Freedman, C. Nayak, K. Shtengel, K. Walker, and Z. Wang, Ann. Phys. 310, 428 (2004) arXiv:cond-mat/0307511.

[25] V. Chari and A. Pressley, A Guide to Quantum Groups (Cambridge University Press, Cambridge, 1994).

[26] C. Kassel, Quantum Groups, Graduate Texts in Mathematics 155 (Springer-Verlag, New York, NY, 1995).

[27] Strictly, here we mean the image of $U\left(\mathrm{sl}_{m}\right)$ in the finitedimensional representations that are trivial under the center $\mathbf{Z}_{m}$ of $\mathrm{SU}(m)$.

[28] J.J. Graham and R.I. Lehrer, Invent. Math. 123, 1 (1996)

[29] A. Mathas, Iwahori-Hecke Algebras and Schur Algebras of the Symmetric Group, University Lecture Series Vol. 10 (American Mathematical Society, Providence, RI, 1999), and references therein.

[30] V.F.R. Jones, L'Enseignement Math. 40, 313 (1994).

[31] K. Erdmann and R.M. Green, arXiv:math.RT/9811017.

[32] P. Martin and H. Saleur, Commun. Math. Phys. 158, 155 (1993).

[33] P. Martin and H. Saleur, Lett. Math. Phys. 30, 189 (1994).

[34] G.H. Hardy and E.M. Wright, An Introduction to the Theory of Numbers, Fifth Edition (Clarendon, Oxford, 1979), Ch. XVI.

[35] The TL algebras for $m$ and $-m$ are isomorphic. Our definition of the algebra by relations (6) differs from some authors by a rescaling by $m$, which makes a difference only in the case $m=0$ (or $\infty$ ).

[36] V. Pasquier and H. Saleur, Nucl. Phys. B 330, 523 (1990).

[37] R. Dipper and G.D. James, Proc. LMS (3), 59, 23 (1989); Trans. AMS 327, 251 (1991).

[38] V.F.R. Jones, in Geometric Methods in Operator Algebras, ed. by H. Araki and E.G. Efros, Pitman Research Notes in Mathematical Sciences Series (Longman Scientific, England, and Wiley, New York, NY, 1986).

[39] J.S. Carter, D.E. Flath, and M. Saito, The Classical and Quantum $6 j$ Symbols (Princeton University, Princeton, NJ, 1995).

[40] B. Bakalov and A. Kirillov, Jr., Lectures on Tensor Categories and Modular Functors, University Lecture Series, No. 21 (American Mathematical Society, Providence, RI, 2001).

[41] V.G. Turaev, Quantum Invariants of Knots and Manifolds, (Walter de Gruyter, Berlin, Germany, 1994).

[42] B. Bakalov and A. Kirillov, Jr., Ref. 40], pp. 35 and 44.

[43] M. Larsen and Z. Wang, Commun. Math. Phys. 260, 641 (2005).

[44] Projective modules [18, 19] are not the same as the "projective representations" which are more familiar to most physicists, and which are not needed here.

[45] See Ref. 19], p. 333.

[46] S. König and C.-C. Xi, in Algebras and Modules II (Geiranger, 1996), Canadian Math. Soc. Conf. Proc. 24 (AMS, Providence, RI, 1998), pp. 365-386; J. Algebra 228, 51 (2000).

[47] C.M. Ringel, Math. Z. 208, 209 (1991).

[48] S. Martin, Schur Algebras and Representation Theory, Cambridge Tracts in Mathematics 112 (Cambridge University, Cambridge, UK, 1993).

[49] J. Du, B. Parshall, L. Scott, Commun. Math. Phys. 195, 321 (1998). 
[50] P.P. Martin and D. Woodcock, J. Phys. A 31, 10131 (1998).

[51] P.P. Martin and B.W. Westbury, J. Phys. A 30, 5471 (1997).

[52] See Ref. [19], Lemma 29.4, p. 324.

[53] P.P. Martin, Ref. 22], Ch. 7.

[54] F.M. Goodman and H. Wenzl, Pac. J. Math. 161, 307 (1993).

[55] P.P. Martin and D. McAnally, Int J. Mod. Phys. A 7, Supp. 1B, 675 (1992).

[56] P.P. Martin, Int J. Mod. Phys. A 7, Supp. 1B, 645 (1992).
[57] N.E. Wegge-Olsen, K-Theory and $C^{*}$-Algebras, (Oxford University, Oxford), App. L, p. 298.

[58] C. Kassel, Ref. [26], Sec. XVI.9, p. 401.

[59] H.Saleur, "Symmetries of the XX chain and applications", in Trieste Conference on Recent developments in Conformal Field Theories (World Scientific, Singapore, 1989).

[60] W.M. Koo and H. Saleur, Nucl. Phys. B 426, 459 (1994).

[61] W.M. Koo and H. Saleur, Int. J. Mod. Phys. A 8, 5165 (1993). 